\title{
Boron Trihalide Mediated Alkyne-Aldehyde Coupling Reactions: A Mechanistic Investigation
}

George W. Kabalka, ${ }^{*}$ Min-Liang Yao, Scott Borella, Zhongzhi Wu, Yu-Hong Ju and Trais Quick

\section{Supporting Information}

\section{Table of Contents}

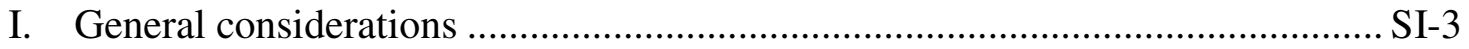

II. Representative procedure for the synthesis of (Z,E)-1,5-dichloro-1,3,5-triaryl-1,4-

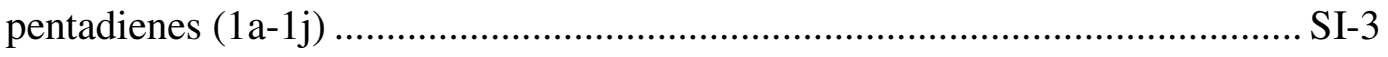

III. Representative procedure for the syntheses of (Z,Z)-1,5-dibromo-1,3,5-triaryl-1,4-

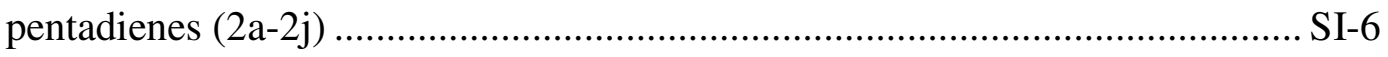

IV. The coupling of alkoxides with vinylboron dihalides to afford dienes 8 (Evidence supporting the migration of halovinyl group from boron to carbon center) ..... SI-8

V. The competition reaction between vinylboron dihalides and allyltrimethylsilane (Evidence supporting cationic mechanism) ................................................... SI-9

VI. Representative procedure for the synthesis of $(Z, Z)-1,5$-dichloro-1,4-pentadienes $(10 \mathrm{a}-\mathrm{k}$ and $11 \mathrm{a}, \mathrm{b})$ SI-9

VII. The $\mathrm{BCl}_{3}$ mediated coupling reaction of allyloxide 5 with phenylacetylene (Evidence supporting the $\mathrm{C}-\mathrm{O}$ bond cleavage in alkoxide)

VIII. Literatures SI-13

\footnotetext{
${ }^{1} \mathrm{H}-\mathrm{NMR}$ spectrum of $1 \mathrm{a}$
} SI-16 
IX. Typical NMR spectra

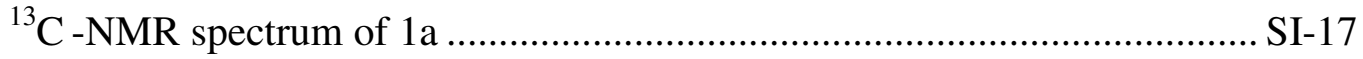

${ }^{13} \mathrm{C}$-NMR spectrum of $1 \mathrm{~b}$............................................................................ SI-18

${ }^{13} \mathrm{C}-\mathrm{NMR}$ spectrum of $1 \mathrm{~b}$........................................................................ SI 19

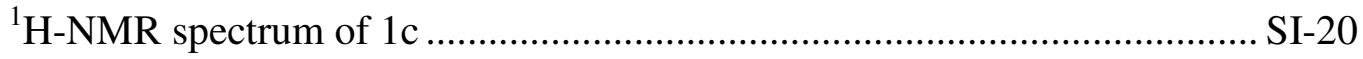

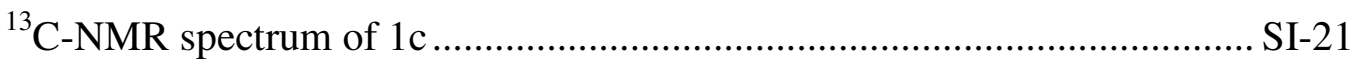

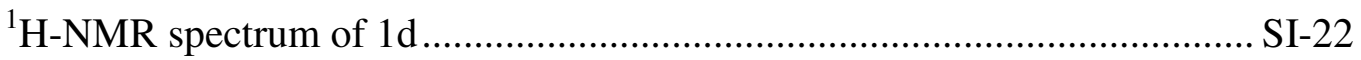

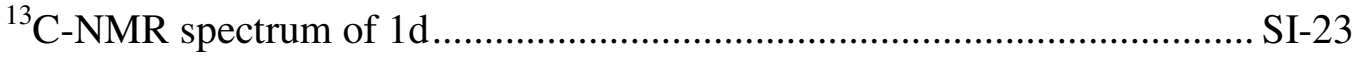

${ }^{1} \mathrm{H}-\mathrm{NMR}$ spectrum of $1 \mathrm{e}$........................................................................... SI-24

${ }^{13} \mathrm{C}$-NMR spectrum of $1 \mathrm{e}$............................................................................ SI-25

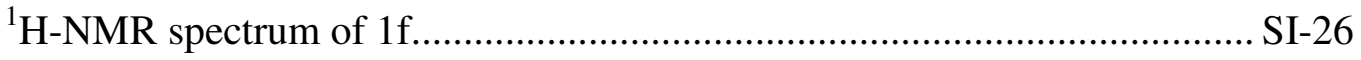

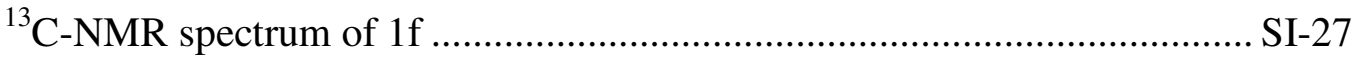

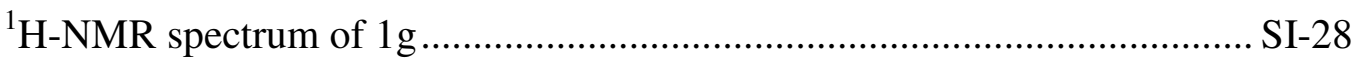

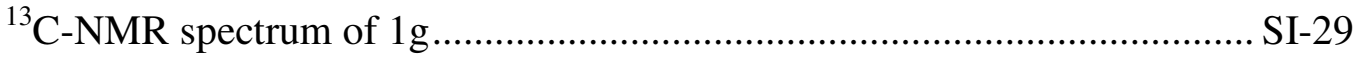

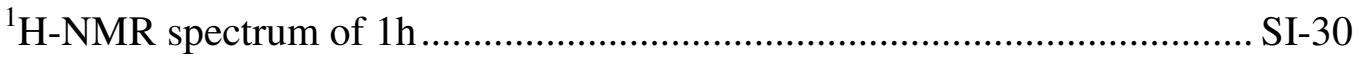

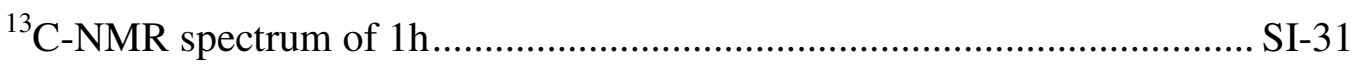

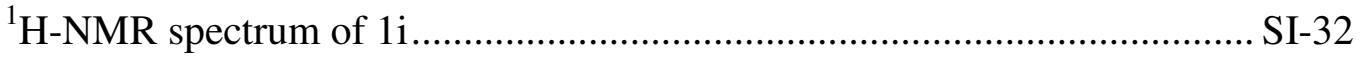

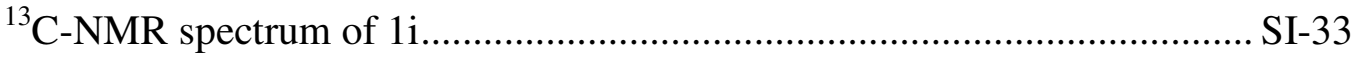

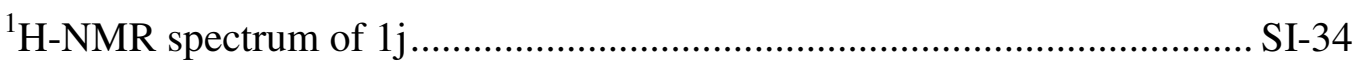

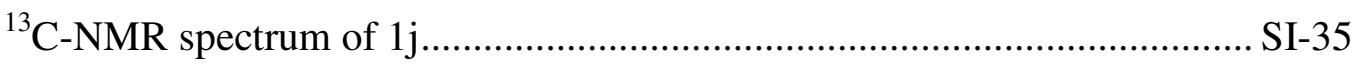

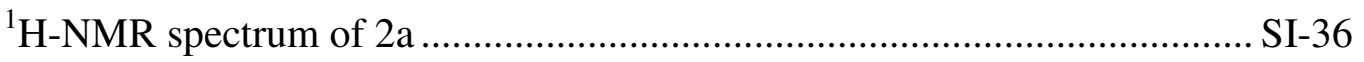

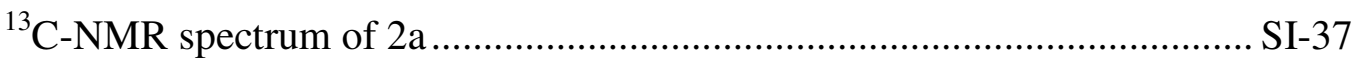

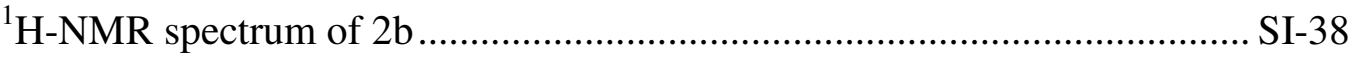

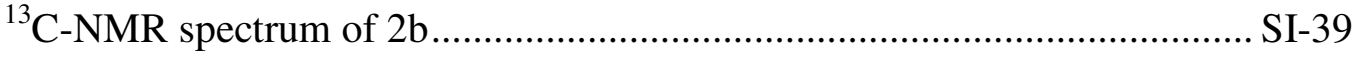

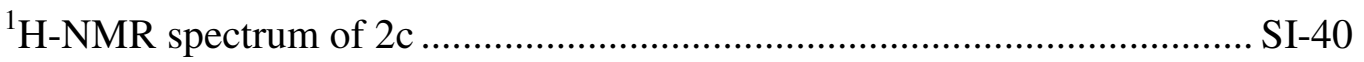

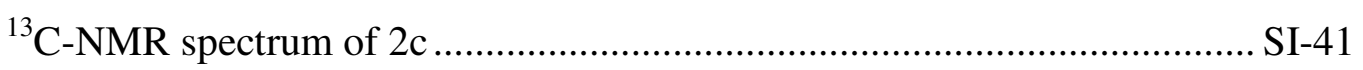

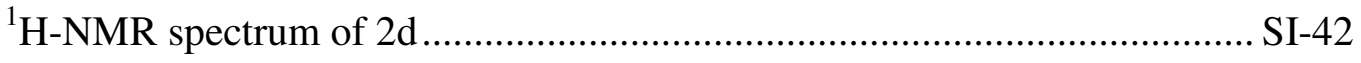

${ }^{13} \mathrm{C}-\mathrm{NMR}$ spectrum of $2 \mathrm{~d}$....................................................................... SI-43

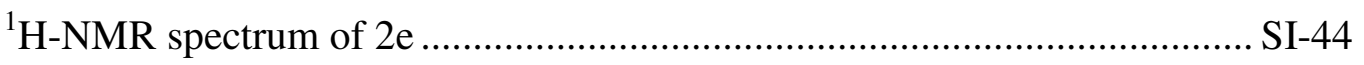




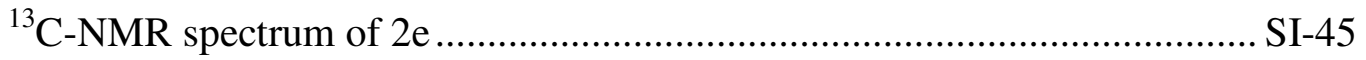

${ }^{1} \mathrm{H}-\mathrm{NMR}$ spectrum of $2 \mathrm{f}$...................................................................... SI-46

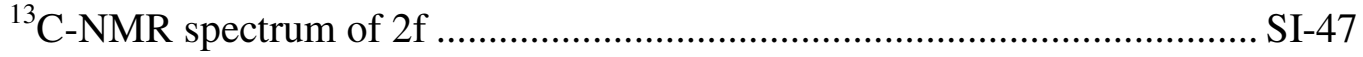

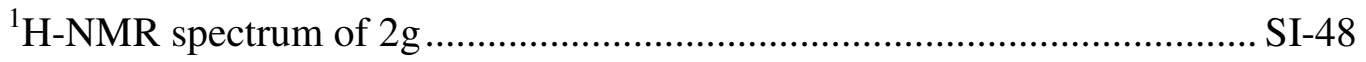

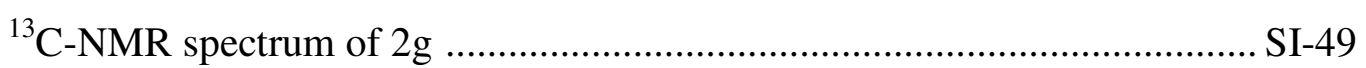

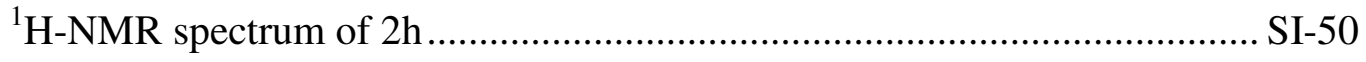

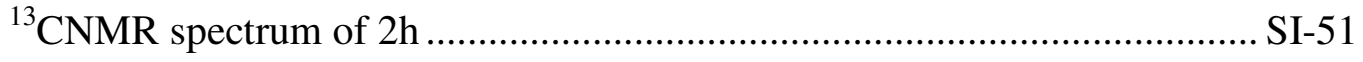

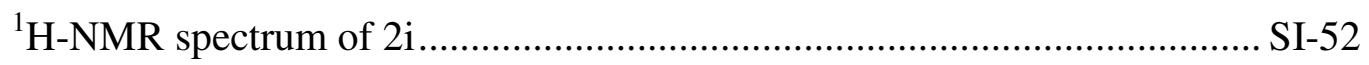

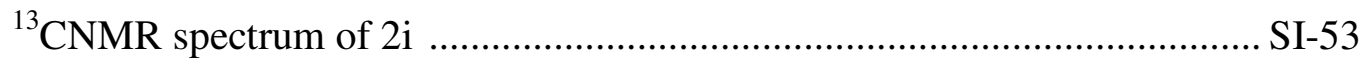

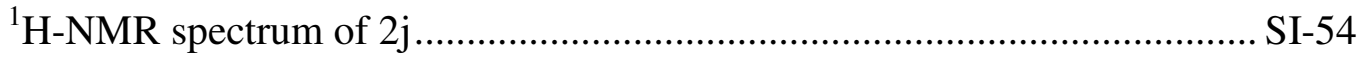

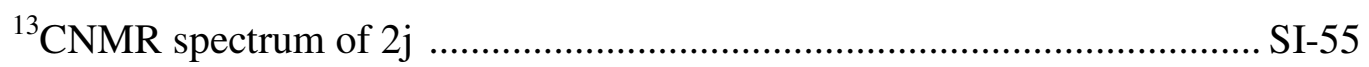

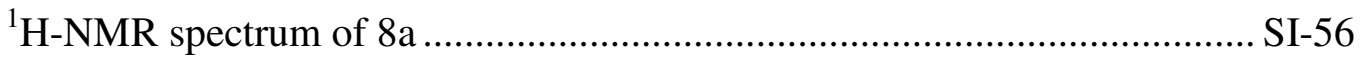

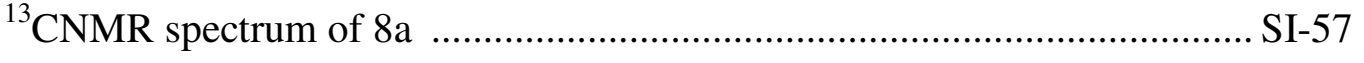

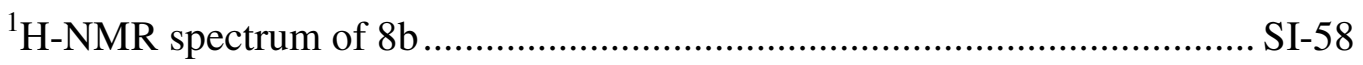

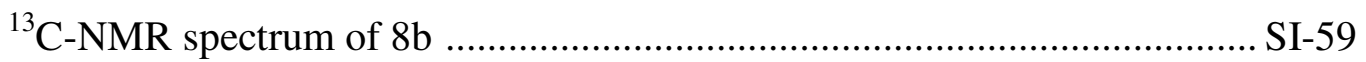

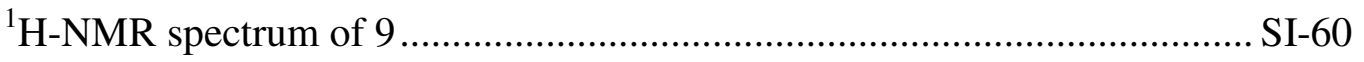

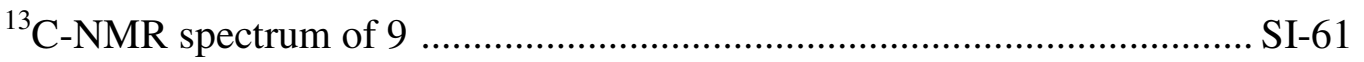

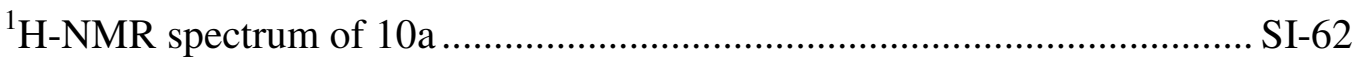

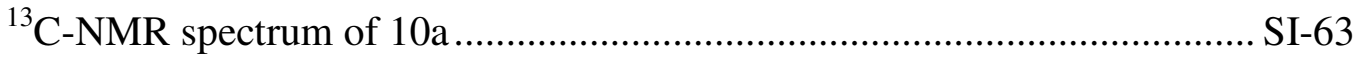

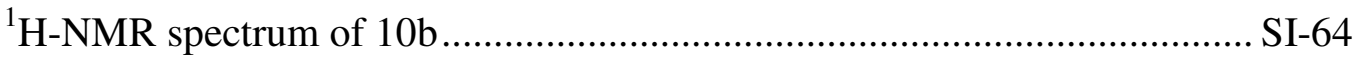

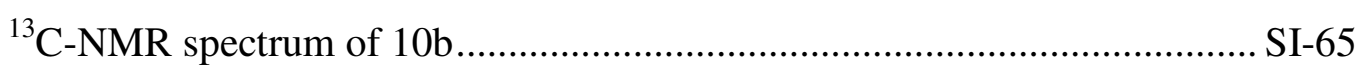

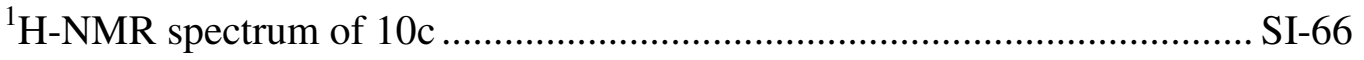

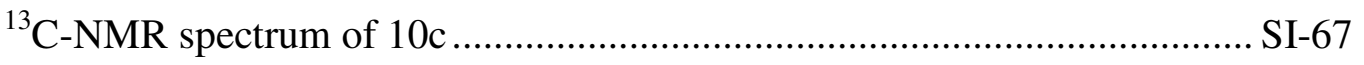

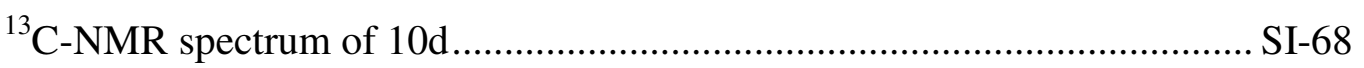

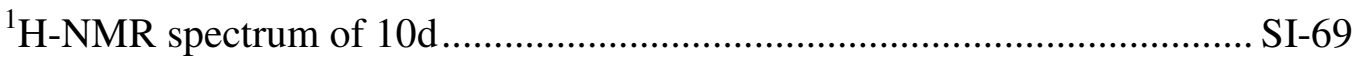

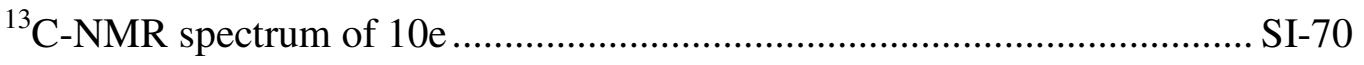

${ }^{1} \mathrm{H}-\mathrm{NMR}$ spectrum of $10 \mathrm{e}$...................................................................... SI-71

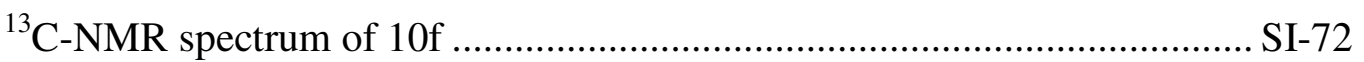

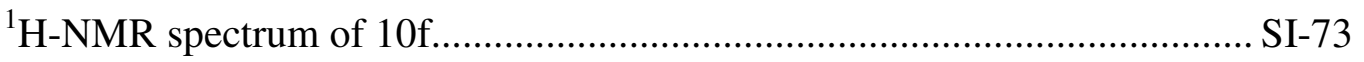

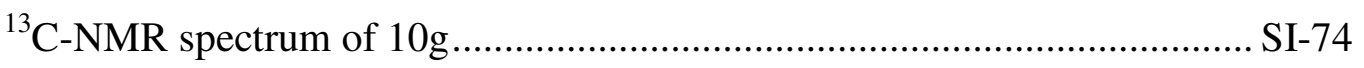

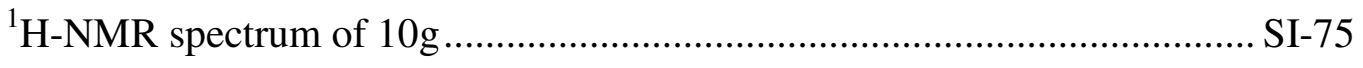




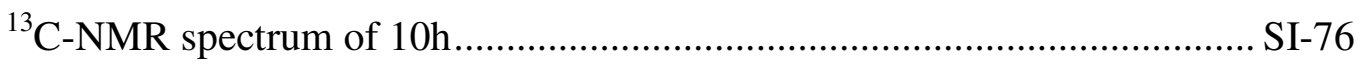

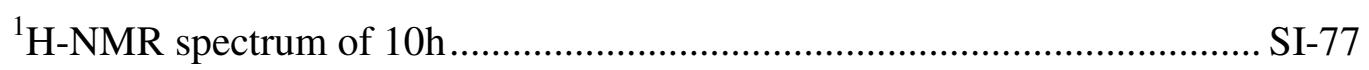

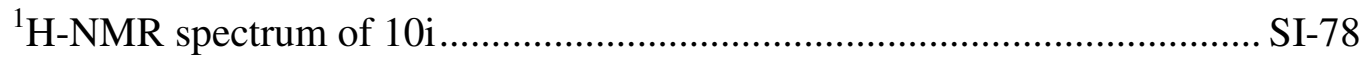

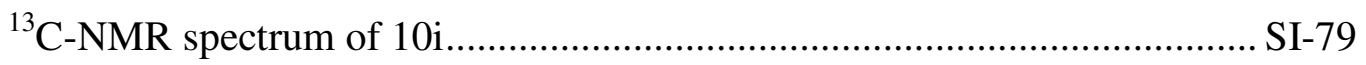

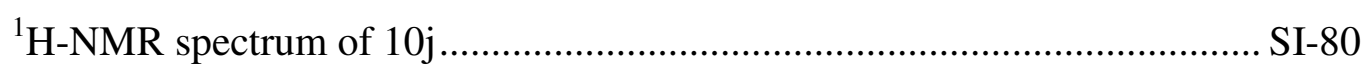

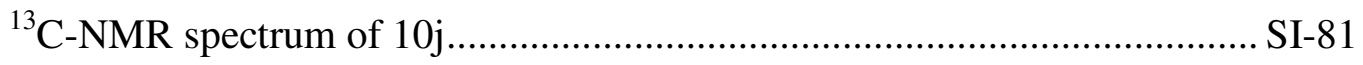

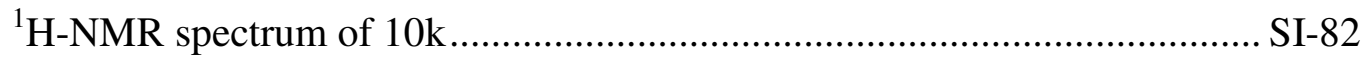

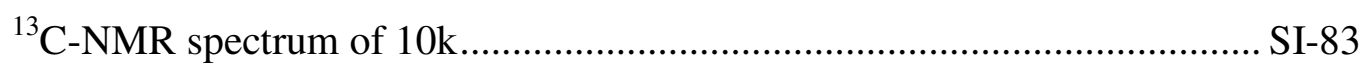

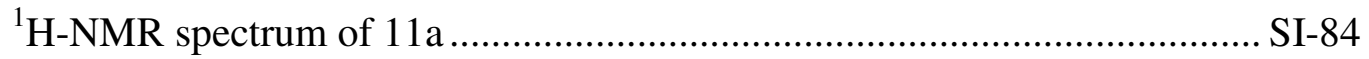

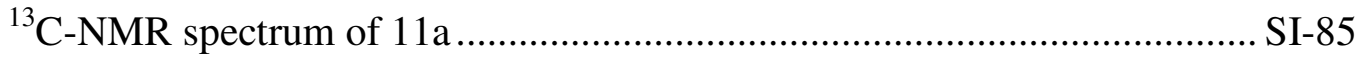

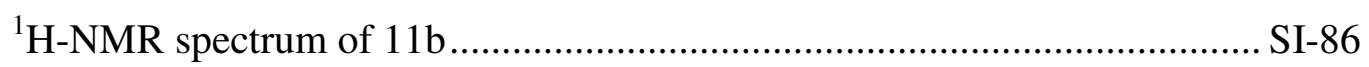

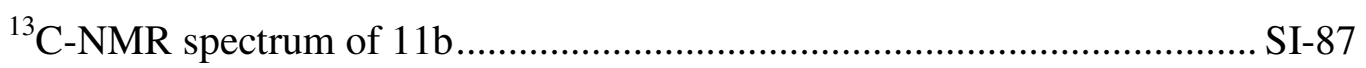

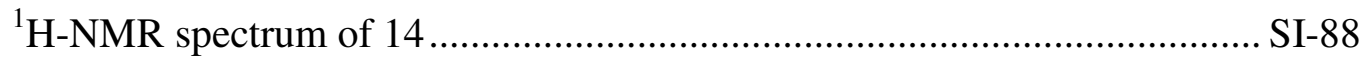

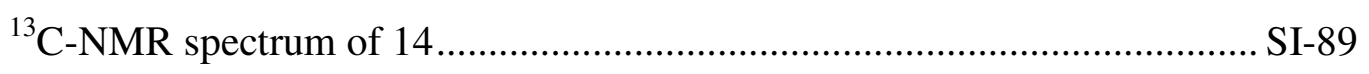


I. General Considerations: All glassware was dried in an oven at $120{ }^{\circ} \mathrm{C}$ and flushed with dry argon. All reactions were carried out under argon atmosphere. $\mathrm{CH}_{2} \mathrm{Cl}_{2}$ was distilled over $\mathrm{CaH}_{2}$. All aldehydes and alkynes were purchased from commercial sources and used as received. Boron trichloride (1M hexane solution) diluted to $1 \mathrm{M}^{\mathrm{CH}_{2} \mathrm{Cl}_{2}}$ solution. Products were purified by flash chromatography using silica gel (60 D, 230-400 mesh). ${ }^{1} \mathrm{H}$ NMR and ${ }^{13} \mathrm{C}$ NMR were recorded in $\mathrm{CDCl}_{3}$ at $250 \mathrm{MHz}$ or $300 \mathrm{MHz}$ with chemical shifts reported relative to TMS.

\section{Representative procedure for the synthesis of $(Z, E)-1,5$-dichloro-1,3,5-triaryl-1,4-}

pentadienes (1a-1j). Benzaldehyde $(0.42 \mathrm{~g}, 4.0 \mathrm{mmol})$ and phenylacetylene $(0.82 \mathrm{~g}, 8.0$ mmol) were placed in a dry argon-flushed, $50 \mathrm{~mL}$ round-bottomed flask equipped with a stirring bar and dissolved in $20 \mathrm{~mL}$ dry $\mathrm{CH}_{2} \mathrm{Cl}_{2}$. The solution was cooled to $0{ }^{\circ} \mathrm{C}$ in an ice bath, and boron trichloride ( $4.4 \mathrm{mmol}, 4.4 \mathrm{~mL}$ of a $1.0 \mathrm{M} \mathrm{CH}_{2} \mathrm{Cl}_{2}$ solution) was added via a syringe. The solution was allowed to stir for $2 \mathrm{hrs}$ at $0{ }^{\circ} \mathrm{C}$ and then for $4 \mathrm{hrs}$ at room temperature. The reaction solution gradually turned dark purple. The resulting mixture was hydrolyzed with water $(20 \mathrm{~mL})$ and extracted with hexanes $(3 \times 30 \mathrm{~mL})$. The organic layer was separated and dried over anhydrous $\mathrm{MgSO}_{4}$. Product $1 \mathrm{a}(0.95 \mathrm{~g}, 65 \%$ yield $)$ was isolated by flash column chromatography.

(Z,E)- 1,5-dichloro-1,3,5-triphenyl-1,4-pentadiene (1a). ${ }^{1} \mathrm{H}$ NMR $(250 \mathrm{MHz}): \delta$ 7.57$7.21(\mathrm{~m}, 15 \mathrm{H}), 6.26(\mathrm{~d}, 1 \mathrm{H}, \mathrm{J}=9.2 \mathrm{~Hz}), 6.19(\mathrm{~d}, 1 \mathrm{H}, \mathrm{J}=10.6 \mathrm{~Hz}), 4.88(\mathrm{dd}, 1 \mathrm{H}, \mathrm{J}=10.6$ and $9.2 \mathrm{~Hz}) .{ }^{13} \mathrm{C}$ NMR: $\delta 141.5,137.6,136.7,133.7,129.0,128.9,128.8,128.3,127.6$, 127.2, 126.9, 126.5, 45.6. Anal. Calcd for $\mathrm{C}_{23} \mathrm{H}_{18} \mathrm{Cl}_{2}$ : C, 75.62; H, 4.97. Found: C, 75.48; $\mathrm{H}, 4.88$. 
(Z,E)-1,5-dichloro-3-(4-fluorophenyl)-1,5-diphenyl-1,4-pentadiene (1b). ${ }^{1} \mathrm{H} \quad \mathrm{NMR}$ (250 MHz): $\delta 7.58-6.99(\mathrm{~m}, 14 \mathrm{H}), 6.22(\mathrm{~d}, 1 \mathrm{H}, \mathrm{J}=9.1 \mathrm{~Hz}), 6.15(\mathrm{~d}, 1 \mathrm{H}, \mathrm{J}=10.4 \mathrm{~Hz})$, $4.85(\mathrm{dd}, 1 \mathrm{H}, \mathrm{J}=10.4$ and $9.1 \mathrm{~Hz}) .{ }^{13} \mathrm{C}$ NMR: $\delta$ 163.7, 159.8, 137.4, 137.1, 136.6, 133.9, 133.3, 129.0, 128.9, 128.8, 128.7, 128.4, 127.3, 126.6, 115.8, 115.5, 44.9. Anal. Calcd for $\mathrm{C}_{23} \mathrm{H}_{17} \mathrm{Cl}_{2} \mathrm{~F}: \mathrm{C}, 72.07 ; \mathrm{H}, 4.47$. Found: $\mathrm{C}, 72.32 ; \mathrm{H}, 4.52$.

(Z,E)-1,5-dichloro-3-(4-bromophenyl)-1,5-diphenyl-1,4-pentadiene (1c). ${ }^{1} \mathrm{H}$ NMR (250 MHz): $\delta$ 7.56- $7.09(\mathrm{~m}, 14 \mathrm{H}), 6.21(\mathrm{~d}, 1 \mathrm{H}, \mathrm{J}=9.1 \mathrm{~Hz}), 6.13(\mathrm{~d}, 1 \mathrm{H}, \mathrm{J}=10.5 \mathrm{~Hz})$, $4.81(\mathrm{dd}, 1 \mathrm{H}, \mathrm{J}=10.5$ and $9.1 \mathrm{~Hz}) .{ }^{13} \mathrm{C}$ NMR: $\delta$ 140.4, 137.3, 136.5, 134.1, 133.5, 131.9, 129.1, 129.0, 128.7, 128.4, 126.9, 126.5, 120.8, 45.0. Anal. Calcd for $\mathrm{C}_{23} \mathrm{H}_{17} \mathrm{BrCl}_{2}$ : C, 62.19; H, 3.86. Found: C, 62.35; H, 3.84.

(Z,E)-1,5-dichloro-1,5-diphenyl-3-(4-methylphenyl)-1,4-pentadiene (1d). ${ }^{1} \mathrm{H} \quad \mathrm{NMR}$ (250 MHz): $\delta 7.58-7.15(\mathrm{~m}, 14 \mathrm{H}), 6.26(\mathrm{~d}, 1 \mathrm{H}, \mathrm{J}=9.2 \mathrm{~Hz}), 6.18(\mathrm{~d}, 1 \mathrm{H}, \mathrm{J}=10.6 \mathrm{~Hz})$, $4.84(\mathrm{dd}, 1 \mathrm{H}, \mathrm{J}=10.6$ and $9.2 \mathrm{~Hz}), 2.33(\mathrm{~s}, 3 \mathrm{H}) .{ }^{13} \mathrm{C}$ NMR: $\delta 138.5,137.6,136.7,136.6$, $133.5,132.8,129.5,129.2,128.9,128.8,128.3,127.8,127.1,126.5,45.2,21.0$. Anal. Calcd for $\mathrm{C}_{24} \mathrm{H}_{20} \mathrm{Cl}_{2}$ : C, 75.99; H, 5.31. Found: C, 76.10; H, 5.40.

(Z,E)-1,5-dichloro-3-(4-cyanophenyl)-1,5-diphenyl-1,4-pentadiene (1e): ${ }^{1} \mathrm{H}$ NMR (250 MHz): $\delta 7.62-7.23(\mathrm{~m}, 14 \mathrm{H}), 6.23(\mathrm{~d}, 1 \mathrm{H}, \mathrm{J}=9.0 \mathrm{~Hz}), 6.15(\mathrm{~d}, 1 \mathrm{H}, \mathrm{J}=10.4 \mathrm{~Hz}), 4.91(\mathrm{dd}$, $1 \mathrm{H}, \mathrm{J}=10.4$ and $9.0 \mathrm{~Hz}) .{ }^{13} \mathrm{C}$ NMR: $\delta 146.8,137.0,136.3,134.9,134.3,132.6,129.2$, 128.5, 128.4, 128.0, 127.5, 126.5, 125.9, 118.6, 110.8, 45.6. Anal. Calcd for $\mathrm{C}_{24} \mathrm{H}_{17} \mathrm{Cl}_{2} \mathrm{~N}$ : C, 73.85; H, 4.39; N, 3.59. Found: C, 73.63; H, 4.42; N, 3.72.

(Z,E)-1,5-dichloro-3-phenyl-1,5-di(4-methylphenyl)-1,4-pentadiene $\quad(1 \mathrm{f}) .{ }^{1} \mathrm{H} \quad \mathrm{NMR}$ (250 MHz): $\delta 7.48-7.12(\mathrm{~m}, 13 \mathrm{H}), 6.22(\mathrm{~d}, 1 \mathrm{H}, \mathrm{J}=9.2 \mathrm{~Hz}), 6.14(\mathrm{~d}, 1 \mathrm{H}, \mathrm{J}=10.6 \mathrm{~Hz})$, $4.87(\mathrm{dd}, 1 \mathrm{H}, \mathrm{J}=10.6$ and $9.2 \mathrm{~Hz}), 2.36(\mathrm{~s}, 3 \mathrm{H}), 2.34(\mathrm{~s}, 3 \mathrm{H}) .{ }^{13} \mathrm{C}$ NMR: $\delta 141.7,138.8$, 
134.9, 133.7, 133.1, 129.0, 128.8, 128.7, 127.3, 126.8, 126.5, 45.6, 21.3, 21.1. Anal. Calcd for $\mathrm{C}_{25} \mathrm{H}_{22} \mathrm{Cl}_{2}$ : C, 76.34; H, 5.64. Found: C, 76.16; H, 5.71.

(Z,E)-1,5-dichloro-3-(4-fluorophenyl)-1,5-di(4-methylphenyl)-1,4-pentadiene (1g). ${ }^{1} \mathrm{H}$ NMR (250 MHz): $\delta 7.46(\mathrm{~d}, 2 \mathrm{H}, \mathrm{J}=8.1 \mathrm{~Hz}), 7.31(\mathrm{~d}, 2 \mathrm{H}, \mathrm{J}=8.1 \mathrm{~Hz}), 7.22-6.95(\mathrm{~m}, 8 \mathrm{H})$, $6.19(\mathrm{~d}, 1 \mathrm{H}, \mathrm{J}=9.0 \mathrm{~Hz}), 6.10(\mathrm{~d}, 1 \mathrm{H}, \mathrm{J}=10.5 \mathrm{~Hz}), 4.84(\mathrm{dd}, 1 \mathrm{H}, \mathrm{J}=10.5$ and $9.0 \mathrm{~Hz})$, 2.36 (s, 3H), 2.35 (s, 3H). ${ }^{13} \mathrm{C}$ NMR: $\delta 163.7,159.8,139.0,137.4,134.7,133.9,133.7$, 133.3, 129.0, 128.8, 128.6, 126.5, 126.4, 115.7, 44.9, 21.3, 21.1. Anal. Calcd for $\mathrm{C}_{25} \mathrm{H}_{21} \mathrm{Cl}_{2} \mathrm{~F}: \mathrm{C}, 73.00 ; \mathrm{H}$, 5.15. Found: C, 72.83; H, 5.29.

(Z,E)-1,5-dichloro-1,3,5-tri(4-methylphenyl)-1,4-pentadiene $\quad(\mathbf{1 h}) . \quad{ }^{1} \mathrm{H} \quad \mathrm{NMR} \quad(250$ MHz): $\delta 7.45(\mathrm{~d}, 2 \mathrm{H}, \mathrm{J}=8.2 \mathrm{~Hz}), 7.33(\mathrm{~d}, 2 \mathrm{H}, \mathrm{J}=8.2 \mathrm{~Hz}), 7.18-7.11(\mathrm{~m}, 8 \mathrm{H}), 6.21(\mathrm{~d}, 1 \mathrm{H}$, $\mathrm{J}=9.1 \mathrm{~Hz}), 6.13(\mathrm{~d}, 1 \mathrm{H}, \mathrm{J}=10.5 \mathrm{~Hz}), 4.84(\mathrm{dd}, 1 \mathrm{H}, \mathrm{J}=10.5$ and $9.1 \mathrm{~Hz}), 2.35-$ 2.31(overlap, 9H). ${ }^{13} \mathrm{C}$ NMR: $\delta 138.8,138.7,136.4,134.9,133.9,133.5,132.8,129.5$, 128.9, 128.7, 127.1, 127.0, 126.4, 45.2, 21.3, 21.0. Anal. Calcd for $\mathrm{C}_{26} \mathrm{H}_{24} \mathrm{Cl}_{2}$ : C, 76.66; H, 5.94. Found: C, 76.62; H, 6.08 .

(Z, E)-1,5-dichloro-1,3,5-tri(4-fluorophenyl)-1,4-pentadiene (1i). ${ }^{1} \mathrm{H}$ NMR (250 MHz): $\delta$ 7.55-7.00 (m, 12H), $6.15(\mathrm{~m}, 2 \mathrm{H}), 4.78(\mathrm{~m}, 1 \mathrm{H}) .{ }^{13} \mathrm{C}$ NMR: $\delta$ 165.0, 164.9, 163.8, $161.1,160.9,159.9,138.9,133.5,132.9,132.7,132.3,130.7,130.6,128.9,128.7,128.5$, 128.3, 127.2, 115.9, 115.6, 115.5, 115.3, 115.2, 44.9. Anal. Calcd for $\mathrm{C}_{23} \mathrm{H}_{15} \mathrm{Cl}_{2} \mathrm{~F}_{3}$ : C, 65.89; H, 3.61. Found: C, 66.13; H, 3.74.

(Z,E)-1,5-dichloro-1,5-di(4-fluorophenyl)-3-(4-methylphenyl)-1,4-pentadiene (1j). ${ }^{1} \mathrm{H}$ NMR (250 MHz): $\delta$ 7.55- 6.99 (m, 12H), $6.17(\mathrm{~m}, 2 \mathrm{H}), 4.76(\mathrm{~m}, 1 \mathrm{H}), 2.33(\mathrm{~s}, 3 \mathrm{H}) .{ }^{13} \mathrm{C}$ NMR: $\delta 165.0,164.8,161.0,160.8,138.2,136.8,133.8,132.8,132.4,131.8,130.8$, 
130.7, 129.6, 129.3, 128.5, 128.3, 127.7, 127.0, 115.5, 115.4, 115.2, 115.1, 45.3, 21.0.

Anal. Calcd for $\mathrm{C}_{24} \mathrm{H}_{18} \mathrm{Cl}_{2} \mathrm{~F}_{2}$ : C, 69.41; H, 4.37. Found: C, 69.64; H, 4.49.

III. Representative procedure for the syntheses of $(Z, Z)-1,5$-dibromo-1,3,5-triaryl1,4-pentadienes (2a-2j). 4-Fluorobenzaldehyde ( $0.52 \mathrm{~g}, 4.2 \mathrm{mmol})$ and phenylacetylene $(0.86 \mathrm{~g}, 8.4 \mathrm{mmol})$ were placed in a dry argon-flushed, $50 \mathrm{~mL}$ round-bottomed flask equipped with a stirring bar and dissolved in $20 \mathrm{~mL}$ dry $\mathrm{CH}_{2} \mathrm{Cl}_{2}$. The solution was cooled to $-40{ }^{\circ} \mathrm{C}$, and boron tribromide $\left(4.5 \mathrm{mmol}, 4.5 \mathrm{~mL}\right.$ of a $1.0 \mathrm{M} \mathrm{CH}_{2} \mathrm{Cl}_{2}$ solution) was added via a syringe. The reaction solution turned purple immediately. The solution was allowed to stir at $-40{ }^{\circ} \mathrm{C}$ for $4 \mathrm{hrs}$. The resulting mixture was hydrolyzed with water (20 $\mathrm{mL})$ and extracted with hexanes $(3 \times 25 \mathrm{~mL})$. The organic layer was separated and dried over anhydrous $\mathrm{MgSO}_{4}$. Product $\mathbf{2 a}(1.80 \mathrm{~g}, 91 \%$ yield) was isolated by flash column chromatography.

(Z,Z)-1,5-Dibromo-1,5-diphenyl-3-(4-fluorophenyl)-1,4-pentadiene (2a). ${ }^{1} \mathrm{H}$ NMR $(250 \mathrm{MHz}): \delta 7.58-7.00(\mathrm{~m}, 14 \mathrm{H}), 6.40(\mathrm{~d}, 2 \mathrm{H}, \mathrm{J}=8.9 \mathrm{~Hz}), 5.31(\mathrm{t}, 1 \mathrm{H}, \mathrm{J}=8.9 \mathrm{~Hz}) .{ }^{13} \mathrm{C}$ NMR: $\delta 163.8,159.8,139.4,136.5,130.7,129.0,128.9,128.3,127.7,127.3,115.8$, 115.5, 50.7. Anal. Calcd for $\mathrm{C}_{23} \mathrm{H}_{17} \mathrm{Br}_{2} \mathrm{~F}: \mathrm{C}, 58.50 ; \mathrm{H}, 3.63$. Found: $\mathrm{C}, 58.41 ; \mathrm{H}, 3.73$.

(Z,Z)-1,5-Dibromo-1,5-diphenyl-3-(4-chlorophenyl)-1,4-pentadiene (2b). ${ }^{1} \mathrm{H}$ NMR (250 MHz): $\delta$ 7.57-7.23 (m, 14H), $6.38(\mathrm{~d}, 2 \mathrm{H}, \mathrm{J}=8.8 \mathrm{~Hz}), 5.30(\mathrm{t}, 1 \mathrm{H}, \mathrm{J}=8.8 \mathrm{~Hz}) .{ }^{13} \mathrm{C}$ NMR: $\delta$ 139.3, 132.7, 130.4, 128.9, 128.8, 128.3, 127.8, 127.7, 127.6, 50.8. Anal. Calcd for $\mathrm{C}_{23} \mathrm{H}_{17} \mathrm{Br}_{2} \mathrm{Cl}$ : C, 56.53; H, 3.51. Found: C, 56.18; H, 3.50.

(Z,Z)-1,5-Dibromo-1,5-diphenyl-3-(4-methylphenyl)-1,4-pentadiene (2c). ${ }^{1} \mathrm{H}$ NMR (250 MHz): $\delta$ 7.58-7.14 (m, 14H), $6.43(\mathrm{~d}, 2 \mathrm{H}, \mathrm{J}=8.9 \mathrm{~Hz}), 5.30(\mathrm{t}, 1 \mathrm{H}, \mathrm{J}=8.9 \mathrm{~Hz}), 2.33$ 
(s, 3H). ${ }^{13} \mathrm{C}$ NMR: $\delta$ 139.6, 137.8, 136.5, 131.2, 129.5, 128.7, 128.2, 127.7, 127.3, 126.8, 51.0, 21.0. Anal. Calcd for $\mathrm{C}_{24} \mathrm{H}_{20} \mathrm{Br}_{2}$ : C, 61.56; H, 4.31. Found: C, 61.83; H, 4.35.

(Z,Z)-1,5-Dibromo-1,5-diphenyl-3-(4-methylphenyl)-1,4-pentadiene (2d). ${ }^{1} \mathrm{H}$ NMR (250 MHz): $\delta 7.55-7.14(\mathrm{~m}, 14 \mathrm{H}), 6.44(\mathrm{~d}, 2 \mathrm{H}, \mathrm{J}=8.7 \mathrm{~Hz}), 5.34(\mathrm{t}, 1 \mathrm{H}, \mathrm{J}=8.7 \mathrm{~Hz}), 2.52$ (s, 3H). ${ }^{13} \mathrm{C}$ NMR: $\delta 140.2,139.4,136.5,131.5,130.8,128.7,128.2,127.7,127.0,126.8$, 126.5, 126.4, 48.8, 20.2. Anal. Calcd for $\mathrm{C}_{24} \mathrm{H}_{20} \mathrm{Br}_{2}$ : C, 61.56; H, 4.31. Found: $\mathrm{C}, 61.27$; H, 4.42 .

(Z,Z)-1,5-Dibromo-1,5-diphenyl-3-(4-nitrophenyl)-1,4-pentadiene (2e). ${ }^{1} \mathrm{H}$ NMR (250 MHz): $\delta$ 8.18- $7.31(\mathrm{~m}, 14 \mathrm{H}), 6.44(\mathrm{~d}, 2 \mathrm{H}, \mathrm{J}=8.8 \mathrm{~Hz}), 5.42(\mathrm{t}, 1 \mathrm{H}, \mathrm{J}=8.8 \mathrm{~Hz}) .{ }^{13} \mathrm{C}$ NMR: $\delta$ 148.2, 146.8, 138.9, 129.3, 129.1, 128.6, 128.4, 127.7, 124.0, 51.3. Anal. Calcd for $\mathrm{C}_{23} \mathrm{H}_{17} \mathrm{Br}_{2} \mathrm{NO}_{2}$ : C, 55.34; $\mathrm{H}, 3.43 ; \mathrm{N}, 2.81$. Found: $\mathrm{C}, 55.60 ; \mathrm{H}, 3.53 ; \mathrm{N}, 2.87$.

(Z,Z)-1,5-Dibromo-1,5-di(4-methylphenyl)-3-(4-fluorophenyl)-1,4-pentadiene (2f). ${ }^{1} \mathrm{H}$ NMR (250 MHz): $\delta$ 7.46- $6.96(\mathrm{~m}, 12 \mathrm{H}), 6.35(\mathrm{~d}, 2 \mathrm{H}, \mathrm{J}=8.9 \mathrm{~Hz}), 5.30(\mathrm{t}, 1 \mathrm{H}, \mathrm{J}=8.9$ $\mathrm{Hz}), 2.31$ (s, 6H). ${ }^{13} \mathrm{C}$ NMR: $\delta 163.6,159.7,138.8,136.5,129.8,129.5,129.3,128.9$, 128.8, 128.2, 127.5, 127.3, 127.1, 115.7, 115.3, 50.6, 21.1. Anal. Calcd for $\mathrm{C}_{25} \mathrm{H}_{21} \mathrm{Br}_{2} \mathrm{~F}$ : C, 60.02; H, 4.23. Found: C, 60.33; H, 4.52.

(Z,Z)-1,5-Dibromo-1,5-di(4-methylphenyl)-3-(4-bromophenyl)-1,4-pentadiene (2g). ${ }^{1} \mathrm{H}$ NMR (250 MHz): $\delta$ 7.45-7.06 (m, 12H), $6.33(\mathrm{~d}, 2 \mathrm{H}, \mathrm{J}=8.9 \mathrm{~Hz}), 5.26(\mathrm{t}, 1 \mathrm{H}, \mathrm{J}=8.9$ Hz), 2.32 (s, 6H). ${ }^{13} \mathrm{C}$ NMR: $\delta 139.9,138.9,136.5,131.8,129.5,128.9,127.5,120.7$, 50.8, 21.1. Anal. Calcd for $\mathrm{C}_{25} \mathrm{H}_{21} \mathrm{Br}_{3}$ : C, 53.51; H, 3.77. Found: C, 53.21; H, 3.99.

(Z,Z)-1,5-Dibromo-1,5-di(4-chlorophenyl)-3-(4-fluorophenyl)-1,4-pentadiene (2h). ${ }^{1} \mathrm{H}$ NMR (250 MHz): $\delta$ 7.51-7.00 (m, 12H), $6.39(\mathrm{~d}, 2 \mathrm{H}, \mathrm{J}=8.9 \mathrm{~Hz}), 5.26(\mathrm{t}, 1 \mathrm{H}, \mathrm{J}=8.9$ Hz). ${ }^{13}$ C NMR: $\delta 163.8,159.9,137.7,136.1,134.8,131.0,128.9,128.8,128.4,126.1$, 
115.9, 115.6, 50.7. Anal. Calcd for $\mathrm{C}_{23} \mathrm{H}_{15} \mathrm{Br}_{2} \mathrm{Cl}_{2} \mathrm{~F}$ : C, 51.05; H, 2.79. Found: C, 51.37; $\mathrm{H}, 2.65$.

(Z,Z)-1,5-Dibromo-1,5-di(4-chlorophenyl)-3-(4-bromophenyl)-1,4-pentadiene (2i). ${ }^{1} \mathrm{H}$ NMR (250 MHz): $\delta$ 7.49-7.20 (m, 12H), $6.37(\mathrm{~d}, 2 \mathrm{H}, \mathrm{J}=8.9 \mathrm{~Hz}), 5.23(\mathrm{t}, 1 \mathrm{H}, \mathrm{J}=8.9$ Hz). ${ }^{13}$ C NMR: $\delta 139.4,137.6,134.8,131.9,130.5,129.1,128.9,128.4,126.4,121.0$, 50.9. Anal. Calcd for $\mathrm{C}_{23} \mathrm{H}_{15} \mathrm{Br}_{3} \mathrm{Cl}_{2}$ : C, 45.89; H, 2.51. Found: C, 46.11; H, 2.66.

(Z,Z)-1,5-Dibromo-1,5-di(4-chlorophenyl)-3-(4-methylphenyl)-1,4-pentadiene $(2 \mathrm{j})$. ${ }^{1} \mathrm{H}$ NMR (250 MHz): $\delta$ 7.46-7.16 (m, 12H), $6.43(\mathrm{~d}, 2 \mathrm{H}, \mathrm{J}=8.7 \mathrm{~Hz}), 5.31(\mathrm{t}, 1 \mathrm{H}, \mathrm{J}=8.7$ $\mathrm{Hz}), 2.51$ (s, 3H). ${ }^{13} \mathrm{C}$ NMR: $\delta 139.7,137.8,136.4,134.6,131.8,130.9,128.9,128.3$, 126.9, 126.5, 125.3, 48.8, 20.2. Anal. Calcd for $\mathrm{C}_{24} \mathrm{H}_{18} \mathrm{Br}_{2} \mathrm{Cl}_{2}: \mathrm{C}, 53.67 ; \mathrm{H}, 3.38$. Found: C, $53.92 ; \mathrm{H}, 3.45$.

\section{The coupling of alkoxides with vinylboron dihalides to afford dienes 8 (Evidence} supporting the migration of halovinyl group from boron to carbon center). Boron trihalide (1.5 mmol, $1.5 \mathrm{~mL}$ of a $1.0 \mathrm{M} \mathrm{CH}_{2} \mathrm{Cl}_{2}$ solution), phenylacetylene $(0.15 \mathrm{~g}, 1.5$ $\mathrm{mmol})$ and dry dichloromethane $(8 \mathrm{~mL})$ were combined in a $50 \mathrm{~mL}$ flask and stirred for 1 hour under a nitrogen atmosphere. Alkoxide 5 (generated in situ by treating $315 \mathrm{mg}, 1.5$ mmol of (Z)-1,3-diphenylprop-2-en-1-ol with $1 \mathrm{~mL}$ of $1.6 \mathrm{M} \mathrm{n}$-BuLi) was added at $0{ }^{\circ} \mathrm{C}$ and allowed to stir at room temperature overnight. Water $(20 \mathrm{~mL})$ was added and the reaction mixture was extracted with ethyl acetate $(3 \times 20 \mathrm{~mL})$ and dried over anhydrous $\mathrm{MgSO}_{4}$. The product was isolated by flash column chromatography using hexane as an eluent.

Spectra for diene 8a (yield: $83 \%) .{ }^{1} \mathrm{H}$ NMR (250 MHz): $\delta$ 7.60-7.57 (m, 2H), 7.37-7.15 (m, 13H), 6.58-6.34 (m, 3H), 4.94 (dd, 1H, J = 9.0 and 8.9 Hz). ${ }^{13} \mathrm{C}$ NMR: $\delta$ 142.1, 137.9, 
137.1, 133.4, 130.9, 130.3, 128.7, 128.5, 128.3, 127.8, 127.4, 126.9, 126.6, 126.3, 48.5.

Anal. Calcd for $\mathrm{C}_{23} \mathrm{H}_{19} \mathrm{Cl}$ : C, 83.50; H, 5.79. Found: C, 83.57; H, 5.65.

Spectra for diene $\mathbf{8 b}$ (yield: $71 \%) .{ }^{1} \mathrm{H}$ NMR $(250 \mathrm{MHz}): \delta 7.59-7.54(\mathrm{~m}, 2 \mathrm{H}), 7.41-7.21$

(m, 13H), 6.55-6.37 (m, 3H), $4.90(\mathrm{dd}, 1 \mathrm{H}, \mathrm{J}=8.94$ and $8.88 \mathrm{~Hz}) .{ }^{13} \mathrm{C}$ NMR: $\delta 141.9$, 139.7, 137.1, 132.1, 131.0, 130.0,128.7, 128.5, 128.3, 127.8, 127.4, 126.8, 126.3, 126.1, 51.3. Anal. Calcd for $\mathrm{C}_{23} \mathrm{H}_{19} \mathrm{Br}$ : C, 73.61; H, 5.10. Found: C, 73.57; H, 5.22.

\section{The competition reaction between vinylboron dihalides and allyltrimethylsilane} (Evidence supporting cationic mechanism). Boron trihalide (1.5 mmol, $1.5 \mathrm{~mL}$ of a 1.0 $M \mathrm{CH}_{2} \mathrm{Cl}_{2}$ solution), phenylacetylene $(153 \mathrm{mg}, 1.5 \mathrm{mmol})$ and dry dichloromethane (8 $\mathrm{mL}$ ) were combined in a $50 \mathrm{~mL}$ flask and stirred for 1 hour under a nitrogen atmosphere. The reaction mixture was cooled to $0{ }^{\circ} \mathrm{C}$, allyltrimethylsilane $(171 \mathrm{mg}, 1.5 \mathrm{mmol})$ and alkoxide 5 (generated in situ by treating $315 \mathrm{mg}, 1.5 \mathrm{mmol}$ of (Z)-1,3-diphenylprop-2-en1-ol with $1 \mathrm{~mL}$ of $1.6 \mathrm{M} n$-BuLi) were added. The resulted reaction mixture was stirred at room temperature overnight. Water $(20 \mathrm{~mL})$ was added and the reaction mixture was extracted with ethyl acetate $(3 \times 20 \mathrm{~mL})$ and dried over anhydrous $\mathrm{MgSO}_{4}$. The product 8a (125 mg, 25\% yield) and 9 (218 mg, 62\% yield) were isolated by flash column chromatography using hexane as an eluent. Compound $\mathbf{9}$ is a known compound. ${ }^{1}$

\section{Representative procedure for the synthesis of (Z,Z)-1,5-dichloro-1,4-pentadienes} (10a-k and 11a, b). Phenylacetylene (306 mg, $3.0 \mathrm{mmol})$ and boron trichloride (1.5 mmol, $1.5 \mathrm{~mL}$ of a $1.0 \mathrm{M} \mathrm{CH}_{2} \mathrm{Cl}_{2}$ solution) were placed in a dry argon-flushed, $50 \mathrm{~mL}$ round-bottomed flask equipped with a stirring bar and dissolved in $20 \mathrm{~mL}$ dry $\mathrm{CH}_{2} \mathrm{Cl}_{2}$. 
The solution was refluxed for $3 \mathrm{hrs}$ and then cooled down to $0{ }^{\circ} \mathrm{C}$ using an ice-bath. To this solution, 4-chlorobenzaldehyde (210 $\mathrm{mg}, 1.5 \mathrm{mmol})$ was added. The solution was allowed to stir for $2 \mathrm{hrs}$ at $0{ }^{\circ} \mathrm{C}$ and then $6 \mathrm{hrs}$ at room temperature. The resulting mixture was hydrolyzed with water $(20 \mathrm{~mL})$ and extracted with hexanes $(3 \times 20 \mathrm{~mL})$. The organic layer was separated and dried over anhydrous $\mathrm{MgSO}_{4}$. Product 10a (471 mg, 78\% yield) was isolated by flash column chromatography.

(Z,Z)-1,5-Dichloro-1,5-diphenyl-3-(4-chlorophenyl)-1,4-pentadiene (10a) ${ }^{1} \mathrm{H} N M R$ (300 MHz): $\delta 7.62-7.29(\mathrm{~m}, 14 \mathrm{H}), 6.29(\mathrm{dd}, 2 \mathrm{H}, \mathrm{J}=9.00$ and $0.9 \mathrm{~Hz}), 5.42(\mathrm{t}, 1 \mathrm{H}, \mathrm{J}=$ $9.00 \mathrm{~Hz}) .{ }^{13} \mathrm{C}$ NMR: $\delta 139.8,137.5,134.8,132.6,128.9,128.8,128.4,126.8,126.6$, 45.2. IR (Neat, $v_{\max } / \mathrm{cm}^{-1}$ ): 3032, 1596, 1491, 1445, 1093, 827, 759, 693. Anal. Calcd for $\mathrm{C}_{23} \mathrm{H}_{17} \mathrm{Cl}_{3}: \mathrm{C}, 69.11 ; \mathrm{H}, 4.29$. Found: C, 69.27; H, 4.31.

(Z,Z)-1,5-Dichloro-1,5-diphenyl-3-(4-bromophenyl)-1,4-pentadiene (10b) ${ }^{1} \mathrm{H}$ NMR $(250 \mathrm{MHz}): \delta 7.78-7.23(\mathrm{~m}, 14 \mathrm{H}), 6.30(\mathrm{~d}, 2 \mathrm{H}, \mathrm{J}=8.97 \mathrm{~Hz}), 5.39(\mathrm{t}, 1 \mathrm{H}, \mathrm{J}=8.97 \mathrm{~Hz})$. ${ }^{13}$ C NMR: $\delta 140.3,137.6,134.8,131.8,129.1,126.7,126.3,126.7,126.6,120.7,45.3$. Anal.Calcd for $\mathrm{C}_{23} \mathrm{H}_{17} \mathrm{BrCl}_{2}$ : C: 62.19; $\mathrm{H}: 3.86$. Found: C: 62.16; H: 3.99 .

(Z,Z)-1,5-Dichloro-1,5-diphenyl-3-(4-cyanophenyl)-1,4-pentadiene (10c). ${ }^{1} \mathrm{H}$ NMR (300 MHz): $\delta 7.65-7.25(\mathrm{~m}, 14 \mathrm{H}), 6.31(\mathrm{dd}, 2 \mathrm{H}, \mathrm{J}=9.0$ and $1.5 \mathrm{~Hz}), 5.49(\mathrm{t}, 1 \mathrm{H}, \mathrm{J}=9.0$ Hz). ${ }^{13}$ C NMR: $\delta 146.8,137.8,137.6,137.3,135.7,132.6,129.1,128.4,128.3,126.6$, 125.8, 118.7, 110.8, 45.8. Anal. Calcd for $\mathrm{C}_{24} \mathrm{H}_{17} \mathrm{Cl}_{2} \mathrm{~N}$ : C, 73.85; H, 4.39; N, 3.59. Found: C, 73.55; H, 4.52; N, 3.56.

(Z,Z)-1,5-Dichloro-1,5-diphenyl-3-(2-methylphenyl)-1,4-pentadiene (10d) ${ }^{1} \mathrm{H}$ NMR (300 MHz): $\delta$ 7.59-7.18 (m, 14H), $6.35(\mathrm{~m}, 2 \mathrm{H}), 5.51(\mathrm{~m}, 1 \mathrm{H}), 2.52(\mathrm{~s}, 3 \mathrm{H}) .{ }^{13} \mathrm{C}$ NMR: $\delta$ 
$140.5,137.7,136.4,133.7,130.8,128.7,128.3,128.0,127.8,126.9,126.8,126.5,126.4$, 43.1, 19.9. HR-MS Calcd for $\mathrm{C}_{24} \mathrm{H}_{20} \mathrm{Cl}_{2}$ : 378.0942. Found: 378.0944 .

(Z,Z)-1,5-Dichloro-1,3,5-triphenyl-1,4-pentadiene (10e) ${ }^{1} \mathrm{H}$ NMR (300 MHz): $\delta$ 7.63-

$7.24(\mathrm{~m}, 15 \mathrm{H}), 6.34(\mathrm{~d}, 2 \mathrm{H}, \mathrm{J}=9.0 \mathrm{~Hz}), 5.47(\mathrm{t}, 1 \mathrm{H}, \mathrm{J}=9.0 \mathrm{~Hz}) .{ }^{13} \mathrm{C}$ NMR: $\delta 141.3$, 137.7, 134.3, 128.8, 128.7, 128.3, 127.4, 127.3, 126.9, 126.6, 45.7. Anal. Calcd for $\mathrm{C}_{23} \mathrm{H}_{18} \mathrm{Cl}_{2}: \mathrm{C}: 75.62 ; \mathrm{H}:$ 4.97. Found: C: 75.33; H: 4.80 .

(Z,Z)-1,5-Dichloro-1,5-di(4-methylphenyl)-3-(4-chlorophenyl)-1,4-pentadiene (10f)

${ }^{1} \mathrm{H}$ NMR (300 MHz): $\delta$ 7.50-7.12 (m, 12H), $6.24(\mathrm{~d}, 2 \mathrm{H}, \mathrm{J}=9.0 \mathrm{~Hz}), 5.39(\mathrm{t}, 1 \mathrm{H}, \mathrm{J}=9.0$

Hz), 2.33 (s, $6 \mathrm{H}) .{ }^{13} \mathrm{C}$ NMR: $\delta$ 140.0, 138.9, 134.7, 132.5, 129.0, 128.8, 128.7, 128.6, 126.5, 126.0, 45.1, 21.1. HR-MS Calcd for $\mathrm{C}_{25} \mathrm{H}_{21} \mathrm{Cl}_{3}$ : 426.0709. Found: 426.0703 .

(Z,Z)-1,5-Dichloro-1,5-di(4-methylphenyl)-3-(4-nitrophenyl)-1,4-pentadiene (10g) ${ }^{1} \mathrm{H}$ NMR (300 MHz): $\delta$ 8.10-7.06 (m, 12H), $6.19(\mathrm{~d}, 2 \mathrm{H}, \mathrm{J}=9.0 \mathrm{~Hz}), 5.42(\mathrm{t}, 1 \mathrm{H}, \mathrm{J}=9.0$ Hz), 2.26 (s, 6H). ${ }^{13} \mathrm{C}$ NMR: $\delta$ 149.1, 146.7, 139.2, 135.7, 134.4, 129.1, 128.3, 126.5, 124.9, 124.0, 53.4, 45.6, 21.1. HR-MS Calcd for $\mathrm{C}_{25} \mathrm{H}_{21} \mathrm{Cl}_{2} \mathrm{NO}_{2}$ : 437.0949. Found: 437.0957.

(Z,Z)-1,5-Dichloro-1,5-di(4-methylphenyl)-3-(3,4-dimethoxyphenyl)-1,4-pentadiene

(10h) ${ }^{1} \mathrm{H}$ NMR (300 MHz): $\delta 7.55-6.82(\mathrm{~m}, 11 \mathrm{H}), 6.27(\mathrm{dd}, 2 \mathrm{H}, \mathrm{J}=9.0$ and $1.5 \mathrm{~Hz}), 5.37$ $(\mathrm{t}, 1 \mathrm{H}, \mathrm{J}=9.0 \mathrm{~Hz}), 3.87(\mathrm{~s}, 3 \mathrm{H}), 3.86(\mathrm{~s}, 3 \mathrm{H}), 2.35(\mathrm{~s}, 6 \mathrm{H}) .{ }^{13} \mathrm{C} \mathrm{NMR}: \delta$ 149.1, 147.9, $138.8,138.2,138.1,138.0,137.9,137.7,135.0,134.1,129.0,126.7,126.5,119.1,111.3$, 110.8, 55.9, 45.3, 21.1. Anal. Calcd for $\mathrm{C}_{27} \mathrm{H}_{26} \mathrm{Cl}_{2} \mathrm{O}_{2}$ : C: 71.52; H: 5.78. Found: C: 70.61; H: 5.80. HR-MS Calcd for $\mathrm{C}_{27} \mathrm{H}_{26} \mathrm{Cl}_{2} \mathrm{O}_{2}$ : 452.1310. Found: 452.1318 .

${ }^{1} \mathrm{H}$ NMR (300 MHz): $\delta$ 7.44-7.24 (m, 12H), $6.28(\mathrm{~d}, 2 \mathrm{H}, \mathrm{J}=9.0 \mathrm{~Hz}), 5.36(\mathrm{t}, 1 \mathrm{H}, \mathrm{J}=9.0$ 
Hz). ${ }^{13}$ C NMR: $\delta$ 139.2, 136.3, 133.8, 132.8, 131.4, 129.0, 128.7, 128.0, 127.0, 123.1, 45.2. IR (Neat, $\left.v_{\max } / \mathrm{cm}^{-1}\right)$ : 3029, 1586, 1486, 1395, 1093, 1073, 1010, 818. Anal.Calcd for $\mathrm{C}_{23} \mathrm{H}_{15} \mathrm{Cl}_{3} \mathrm{Br}_{2}: \mathrm{C}: 49.55 ; \mathrm{H}: 2.71$. Found: C: $49.23 ; \mathrm{H}: 2.67$.

(Z,Z)-1,5-Dichloro-1,5-di(3-methoxyphenyl)-3-(4-trifluoromethylphenyl)-1,4-pentadiene (10j) ${ }^{1} \mathrm{H}$ NMR $(300 \mathrm{MHz}): \delta$ 7.61-6.87 (m, 12H), $6.33(\mathrm{dd}, 2 \mathrm{H}, \mathrm{J}=9.0$ and 0.6 $\mathrm{Hz}), 5.50(\mathrm{t}, 1 \mathrm{H}, \mathrm{J}=9.0 \mathrm{~Hz}), 3.82(\mathrm{~s}, 6 \mathrm{H}) .{ }^{13} \mathrm{C}$ NMR: $\delta 159.5,145.3,138.9,135.0,129.4$, $127.8,126.6,125.8,125.7,119.0,114.5,112.5,55.3,45.6$. HR-MS Calcd for $\mathrm{C}_{26} \mathrm{H}_{21} \mathrm{Cl}_{2} \mathrm{~F}_{3} \mathrm{O}_{2}: 492.0871$. Found: 492.0879 .

(Z,Z)-1,5-Dichloro-1,5-di(3-fluorophenyl)-3-(4-chlorophenyl)-1,4-pentadiene (10k) ${ }^{1} \mathrm{H}$ NMR(300 MHz): $\delta$ 7.41-7.00 (m, 12H), $6.33(\mathrm{~d}, 2 \mathrm{H}, \mathrm{J}=9.0 \mathrm{~Hz}), 5.39(\mathrm{t}, 1 \mathrm{H}, \mathrm{J}=9.0$ Hz). ${ }^{13}$ C NMR: $\delta 164.2,161.0,139.6,139.2,138.2,138.0,137.9,133.6,133.5,132.9$, 129.9, 129.8, 129.0, 128.7, 127.6, 122.2, 122.1, 116.0, 115.7, 113.9, 113.6, 45.2. Anal. Calcd for $\mathrm{C}_{23} \mathrm{H}_{15} \mathrm{Cl}_{2} \mathrm{~F}_{2}$ : C, 63.40; H, 3.47. Found: C, 63.51; H, 3.29.

(Z,Z)-1,5-Dichloro-1,5-diphenyl-1,4-pentadiene (11a) ${ }^{1} \mathrm{H}$ NMR $(250 \mathrm{MHz}): \delta$ 7.61$7.25(\mathrm{~m}, 10 \mathrm{H}), 6.21(\mathrm{t}, 2 \mathrm{H}, \mathrm{J}=7.2 \mathrm{~Hz}), 3.50(\mathrm{t}, 2 \mathrm{H}, \mathrm{J}=7.2 \mathrm{~Hz}) .{ }^{13} \mathrm{C} \mathrm{NMR}: \delta 137.9$, 134.4, 128.6, 128.3, 126.5, 123.8, 30.2. HR-MS Calcd for $\mathrm{C}_{17} \mathrm{H}_{14} \mathrm{Cl}_{2}$ : 288.0473. Found: 288.0477 .

(Z,Z)-1,5-Dichloro-1,5-diphenyl-3-hexyl-1,4-pentadiene (11b) ${ }^{1} \mathrm{H}$ NMR (300 MHz): $\delta$ 7.60-7.29 (m, 10H), $6.01(\mathrm{dd}, 2 \mathrm{H}, \mathrm{J}=9.0$ and $1.2 \mathrm{~Hz}), 4.13(\mathrm{~m}, 1 \mathrm{H}), 1.63-1.58(\mathrm{~m}, 2 \mathrm{H})$, 1.34-1.26 (m, 8H), $0.88(\mathrm{t}, 3 \mathrm{H}, \mathrm{J}=6.2 \mathrm{~Hz}) .{ }^{13} \mathrm{C}$ NMR: $\delta$ 138.1, 133.3, 128.8, 128.5, 128.2, 126.5, 40.9, 34.9, 31.8, 29.3, 26.9, 22.6, 14.1. HR-MS Calcd for $\mathrm{C}_{23} \mathrm{H}_{26} \mathrm{Cl}_{2}$ : 372.1412. Found: 372.1420. 


\section{The $\mathrm{BCl}_{3}$ mediated coupling reaction of allyloxide 5 with phenylacetylene}

(Evidence supporting the $\mathrm{C}-\mathrm{O}$ bond cleavage in alkoxide). Alkoxide $\mathbf{5}$ (generated in situ by treating $315 \mathrm{mg}, 1.5 \mathrm{mmol}$ of (Z)-1,3-diphenylprop-2-en-1-ol with $1 \mathrm{~mL}$ of $1.6 \mathrm{M}$ $n$-BuLi), phenylacetylene (153 $\mathrm{mg}, 1.5 \mathrm{mmol})$ and dry dichloromethane $(8 \mathrm{~mL})$ were combined in a $50 \mathrm{~mL}$ flask under a nitrogen atmosphere at $0^{\circ} \mathrm{C}$. Boron trihalide $(1.5$ mmol, $1.5 \mathrm{~mL}$ of a $1.0 \mathrm{M} \mathrm{CH}_{2} \mathrm{Cl}_{2}$ solution) was added and stirred at room temperature overnight. Water $(20 \mathrm{~mL})$ was added and the reaction mixture was extracted with ethyl acetate $(3 \times 20 \mathrm{~mL})$ and dried over anhydrous $\mathrm{MgSO}_{4}$. Product 14 (275 mg, $78 \%$ yield) was isolated by flash column chromatography using hexane as an eluent. Spectra for

diene (E, E)-1-Chloro-1,3,5-triphenyl-1,4-pentadiene (14). ${ }^{1} \mathrm{H}$ NMR (250 $\left.\mathrm{MHz}, \mathrm{CDCl}_{3}\right)$ : $\delta$ 7.44-7.17 (m, 15H), 6.48-6.20 (m, 3H), $4.32(\mathrm{dd}, 1 \mathrm{H}, J=10.6$ and $10.5 \mathrm{~Hz}) .{ }^{13} \mathrm{C} \mathrm{NMR}$ $\left(\mathrm{CDCl}_{3}\right): \delta 142.3,136.9,131.6,131.2,130.8,130.4,128.9,128.8,128.6,128.4,127.6$, 127.5, 126.8, 126.3, 48.4. Anal. Calcd for $\mathrm{C}_{23} \mathrm{H}_{19} \mathrm{Cl}$ : C, 83.50; H, 5.79. Found: C, 83.44; H, 5.82 .

\section{Literature}

1. Kaur, G.; Kaushik, M.; Trehan, S. Tetrahedron Lett. 1997, 38. 2521. 


\section{${ }^{1} \mathrm{H}$ NMR Spectrum of 1a}
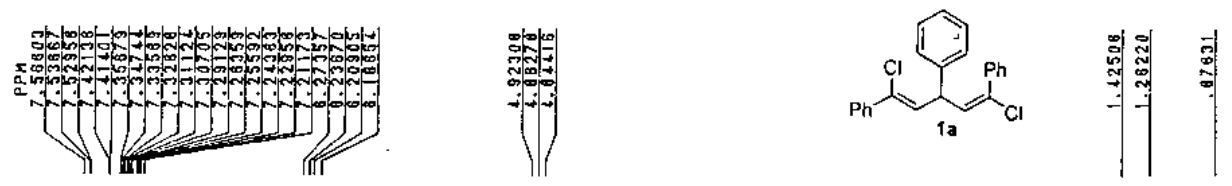

임

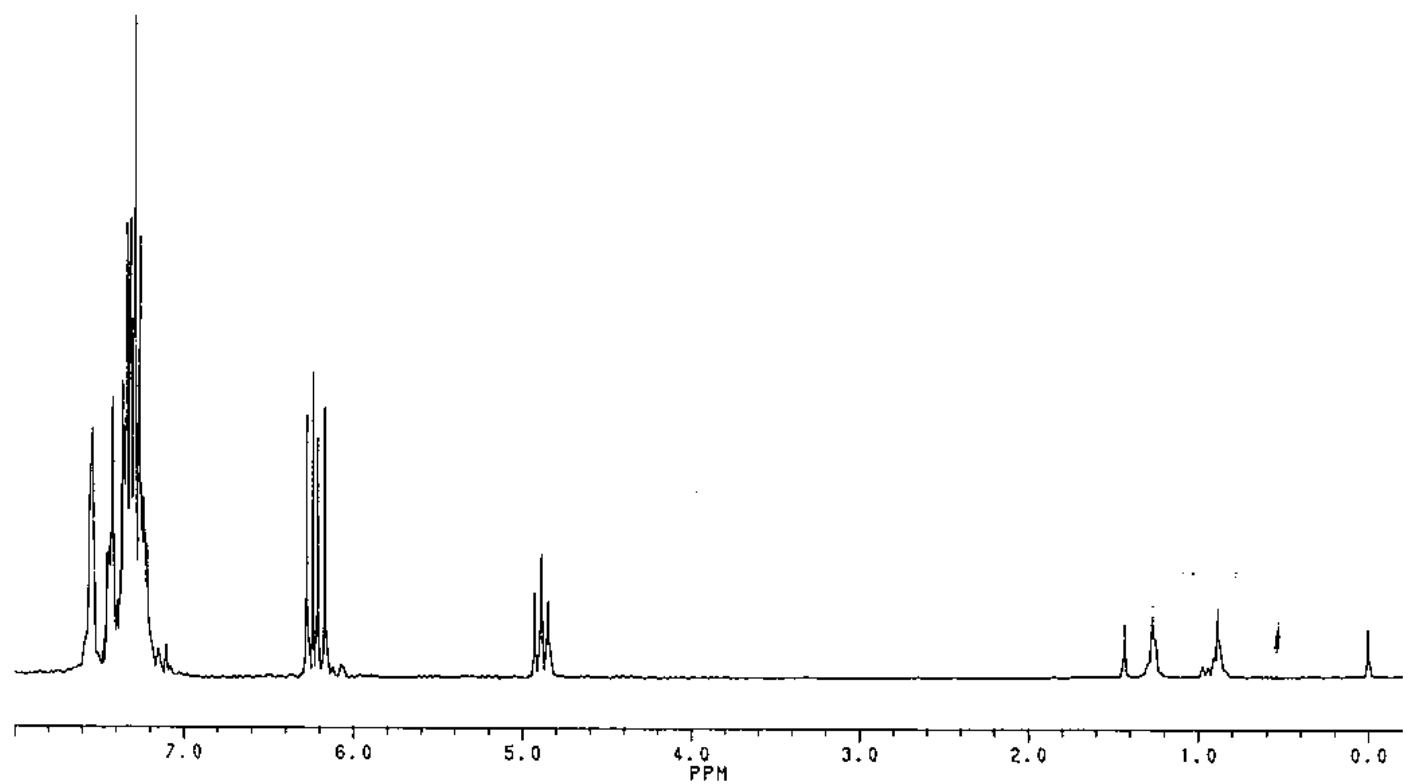




\section{${ }^{13} \mathrm{C}$ NMR Spectrum of $1 \mathrm{a}$}
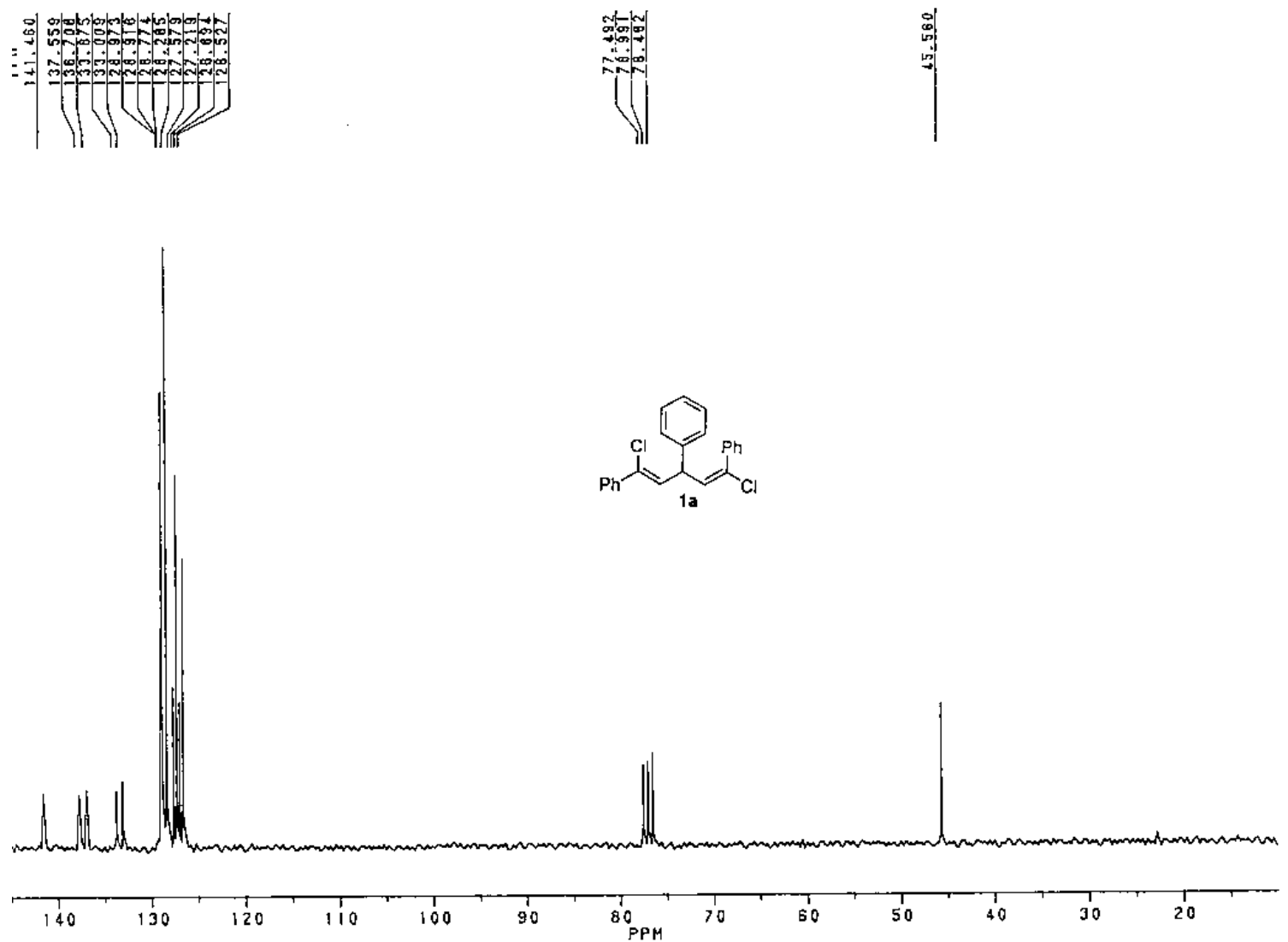


\section{${ }^{1} \mathrm{H}$ - NMR spectrum of $1 \mathrm{~b}$}
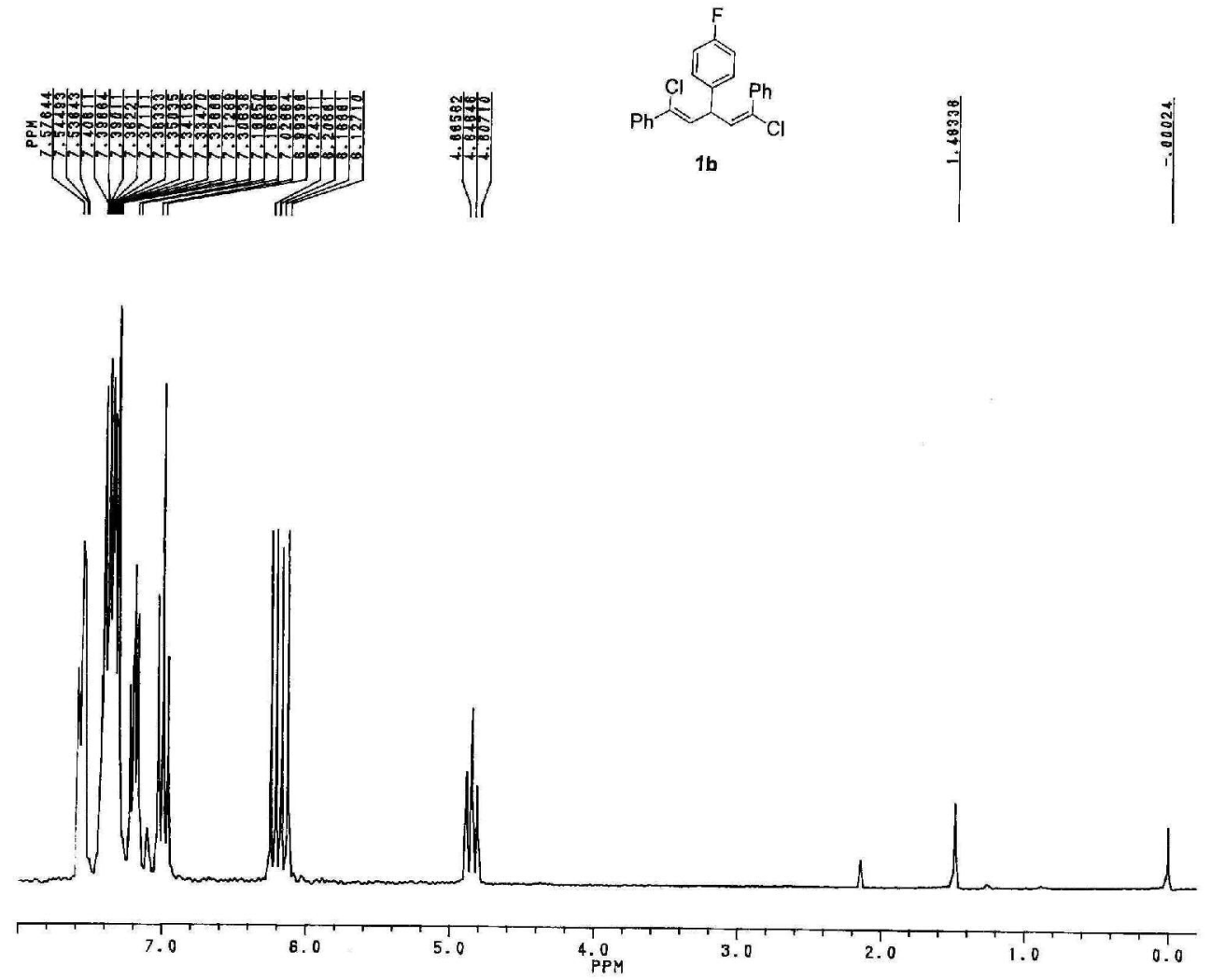

SI-18 


\section{${ }^{13} \mathrm{C}$-NMR spectrum of $1 \mathrm{~b}$}

출
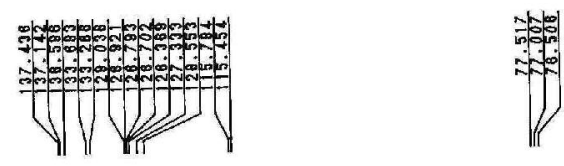

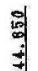

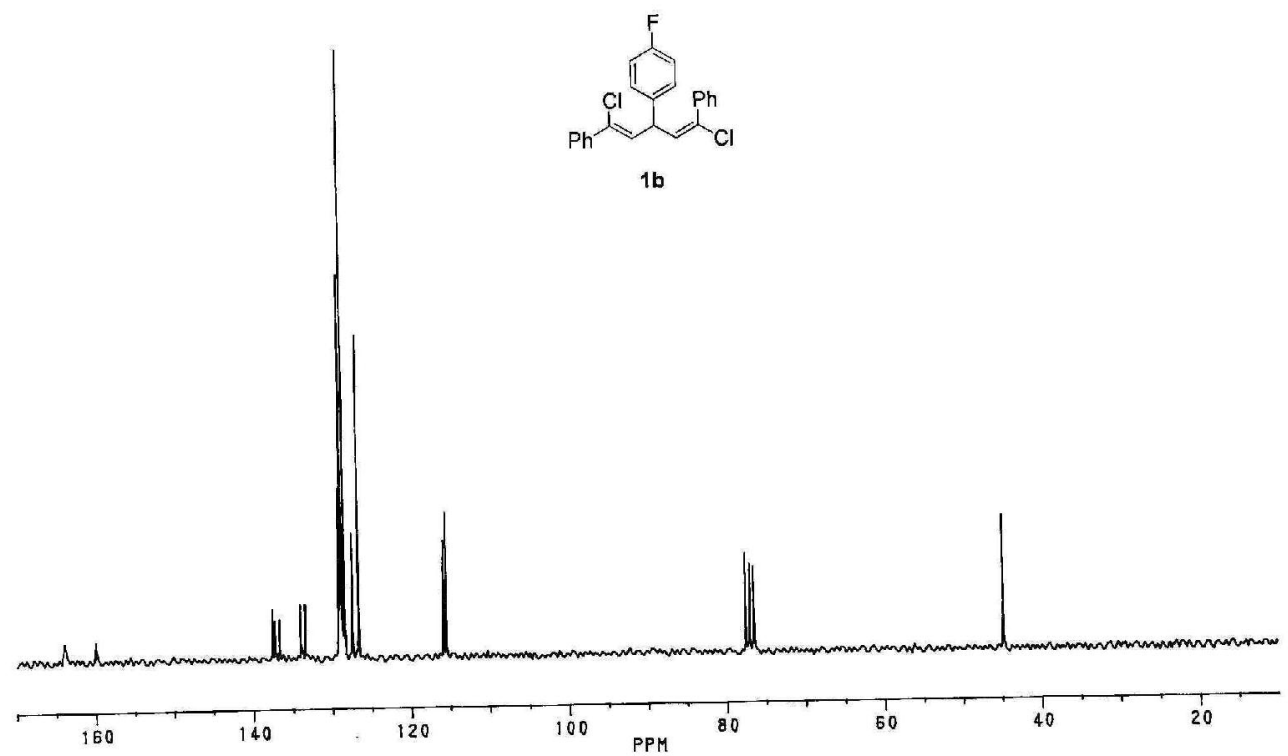




\section{${ }^{1} \mathrm{H}$ NMR spectrum of $1 \mathrm{c}$}
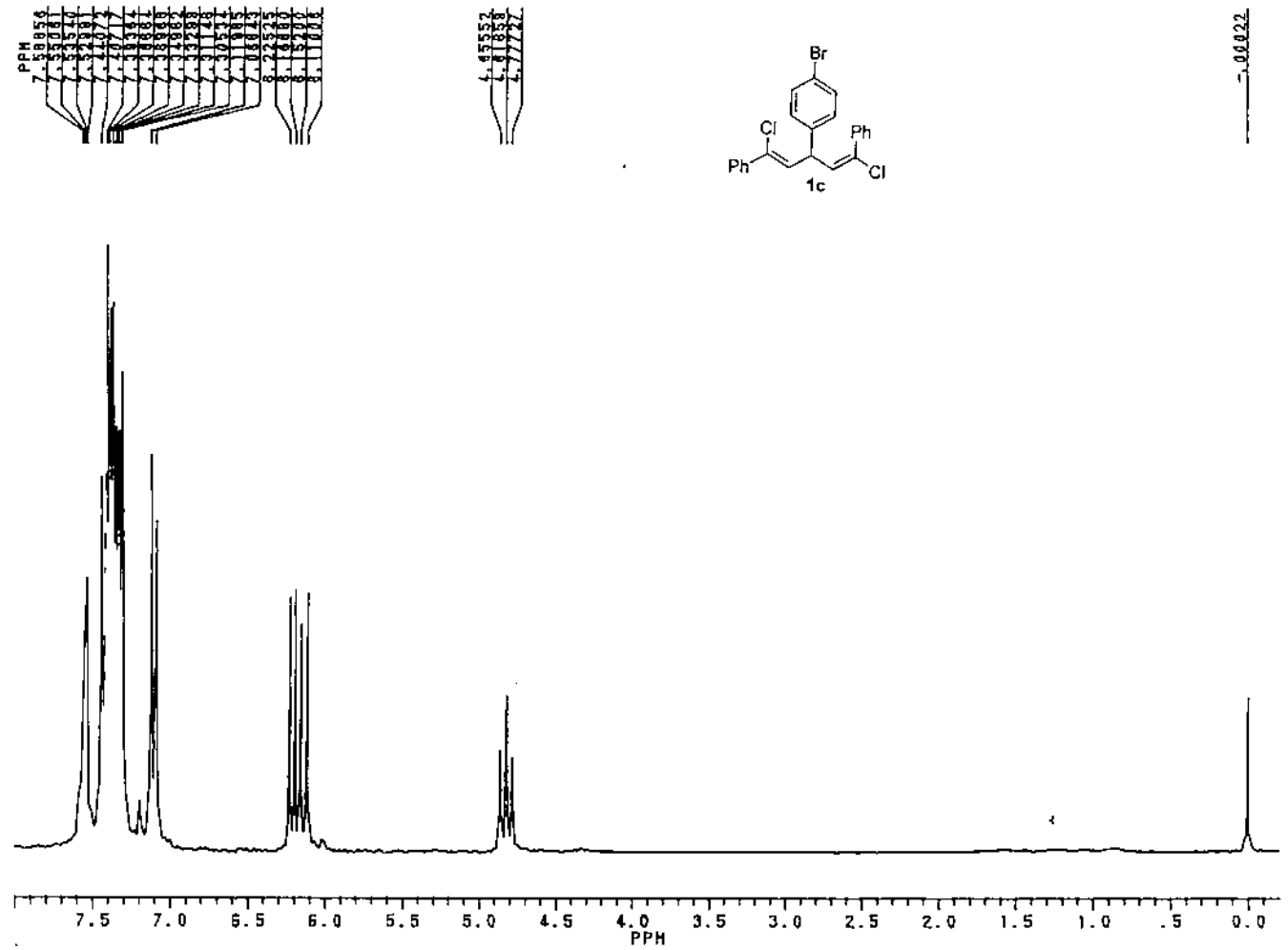


\section{${ }^{13} \mathrm{C}$ NMR spectrum of $1 \mathrm{c}$}

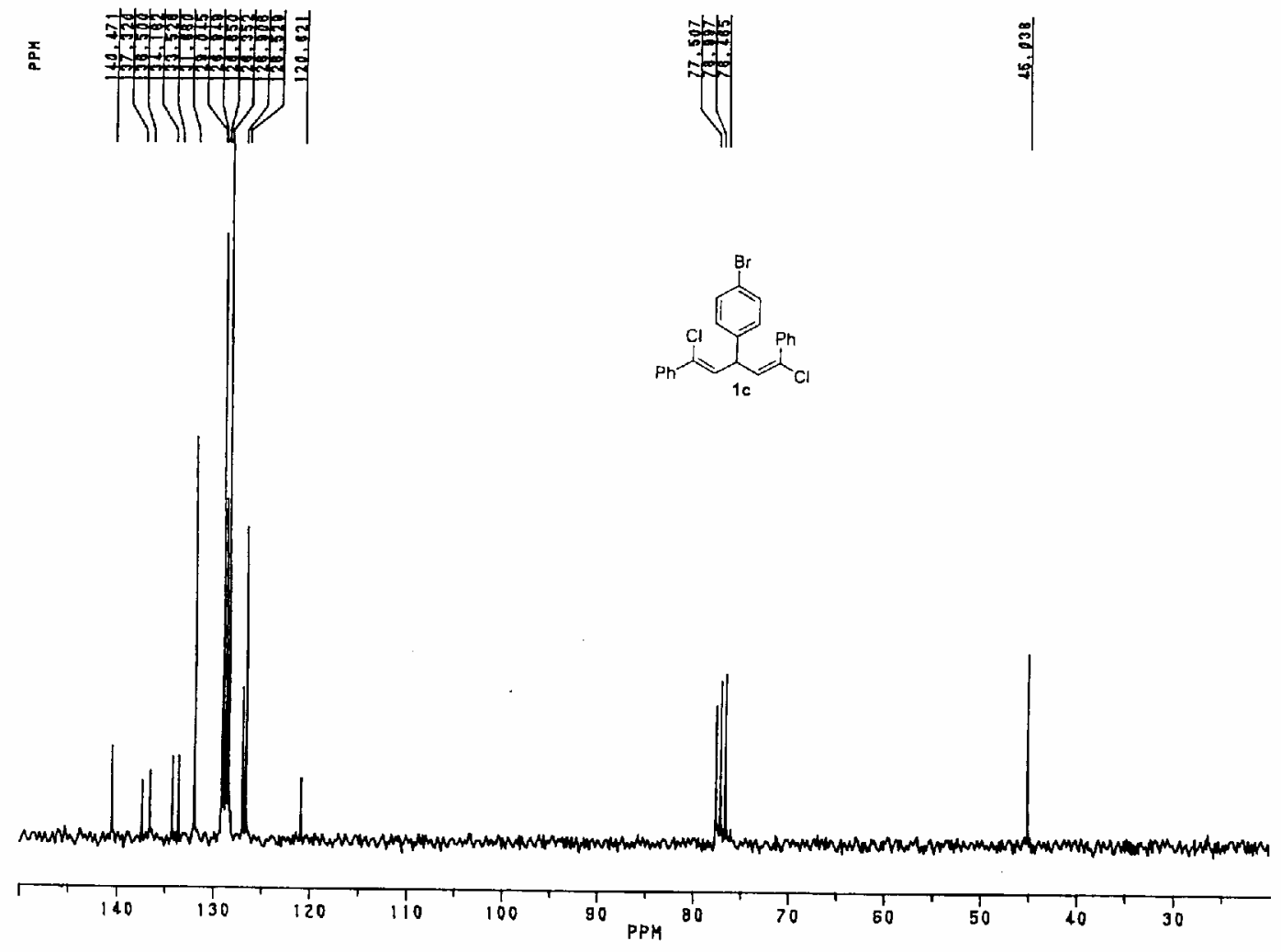




\section{${ }^{1} \mathrm{H}$ NMR spectrum for $1 \mathrm{~d}$}
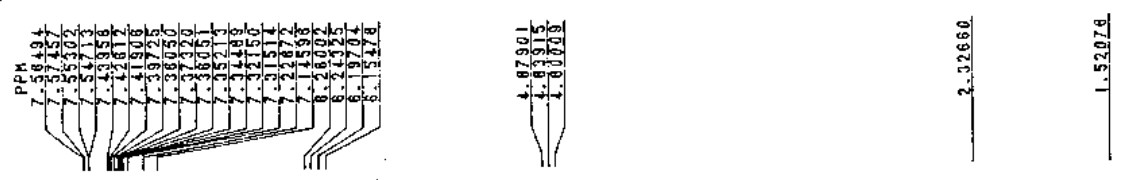

:

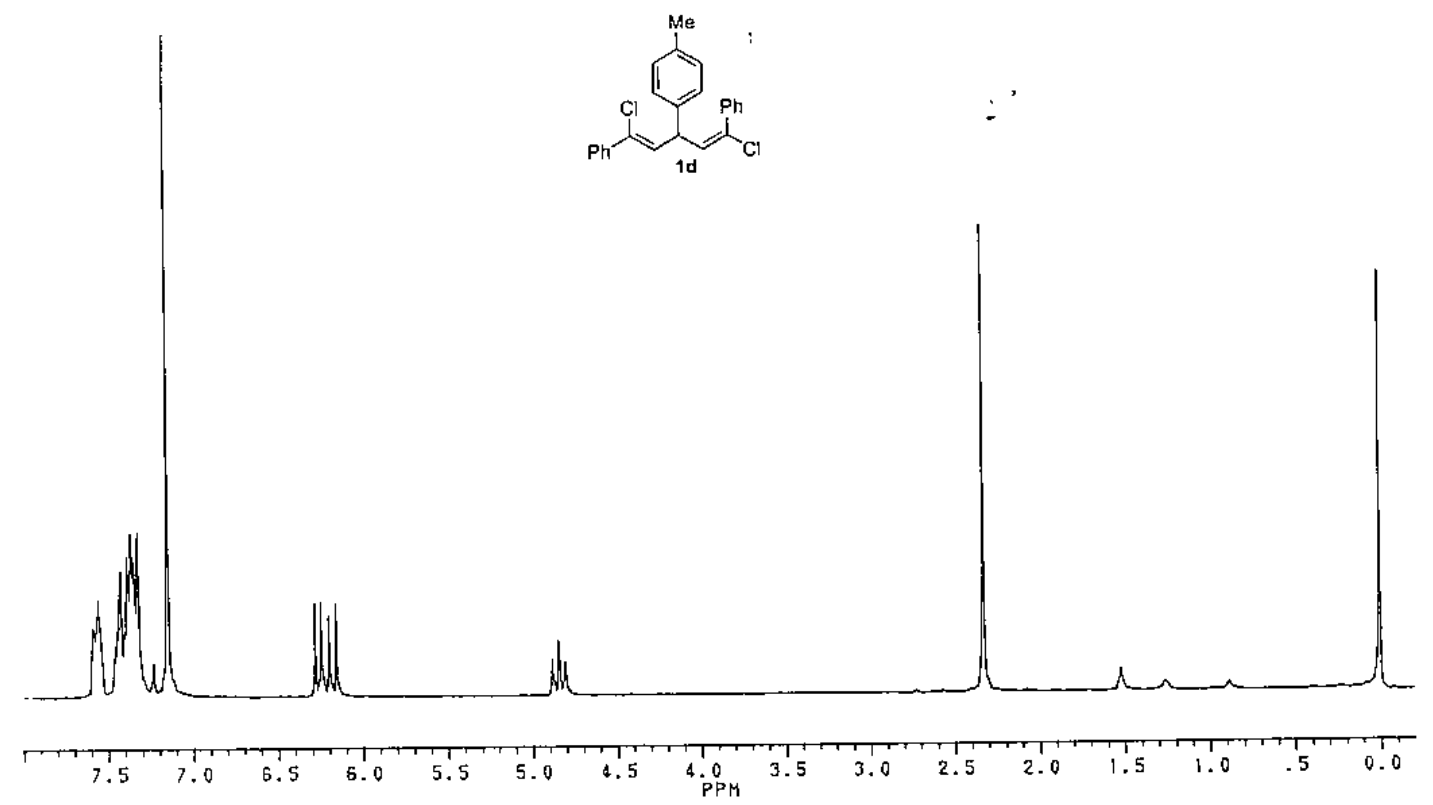




\section{${ }^{13} \mathrm{C}$ NMR spectrum of $1 \mathrm{~d}$}
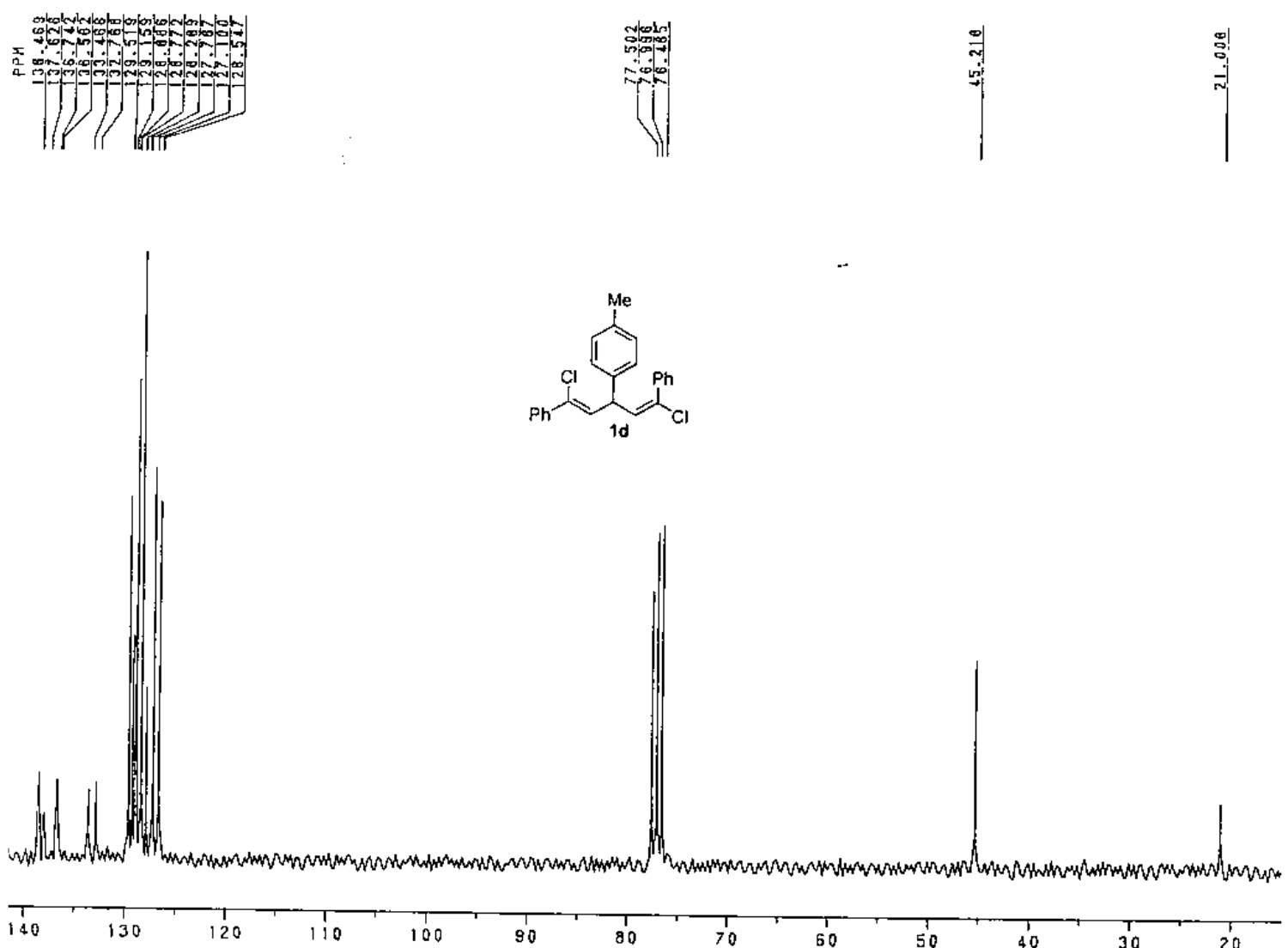

9

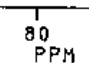




\section{${ }^{1} \mathrm{H}$ - NMR spectrum of $1 \mathrm{e}$}

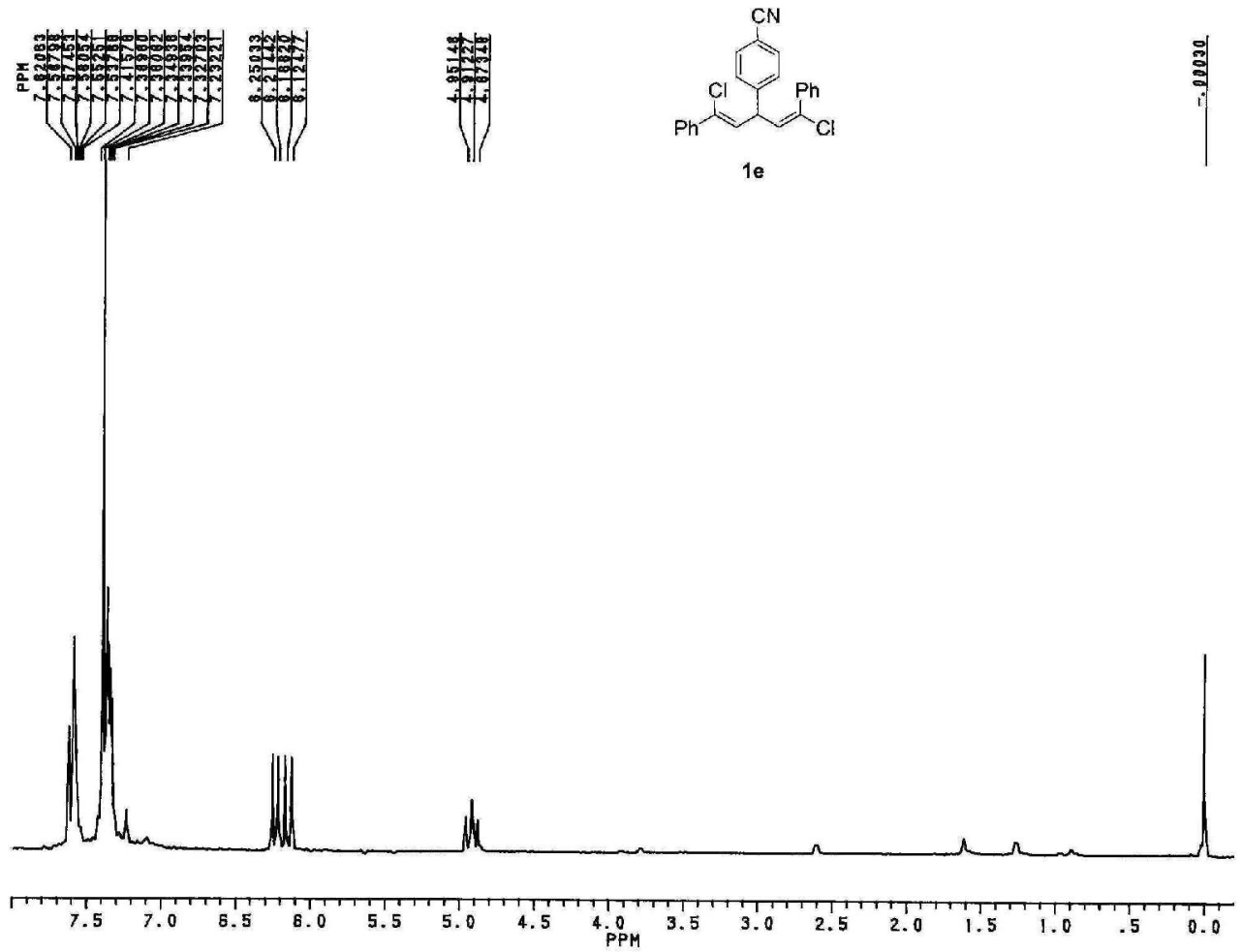




\section{${ }^{13} \mathrm{C}$-NMR spectrum of $1 \mathrm{e}$}

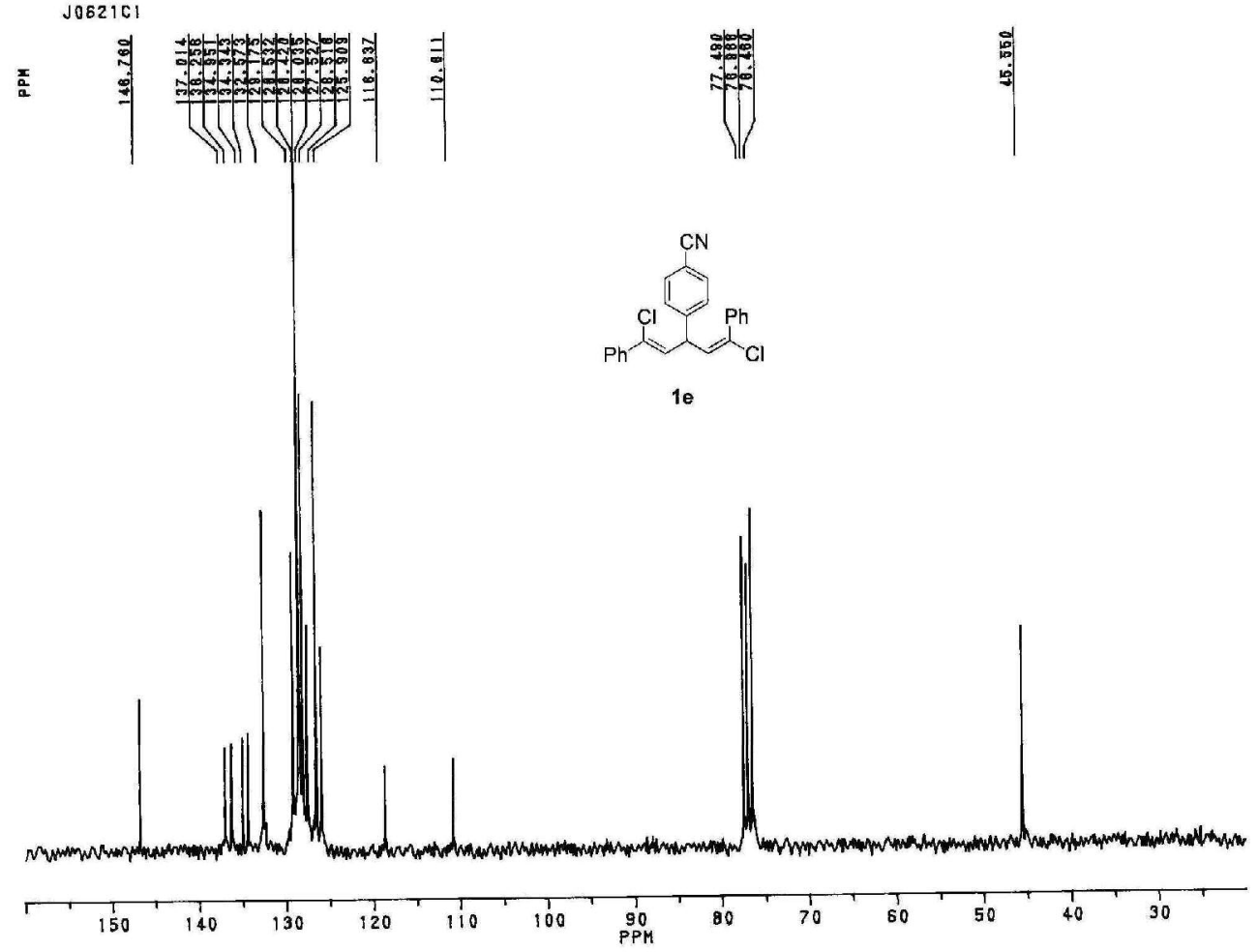




\section{${ }^{1}$ H- NMR spectrum of $1 f$}
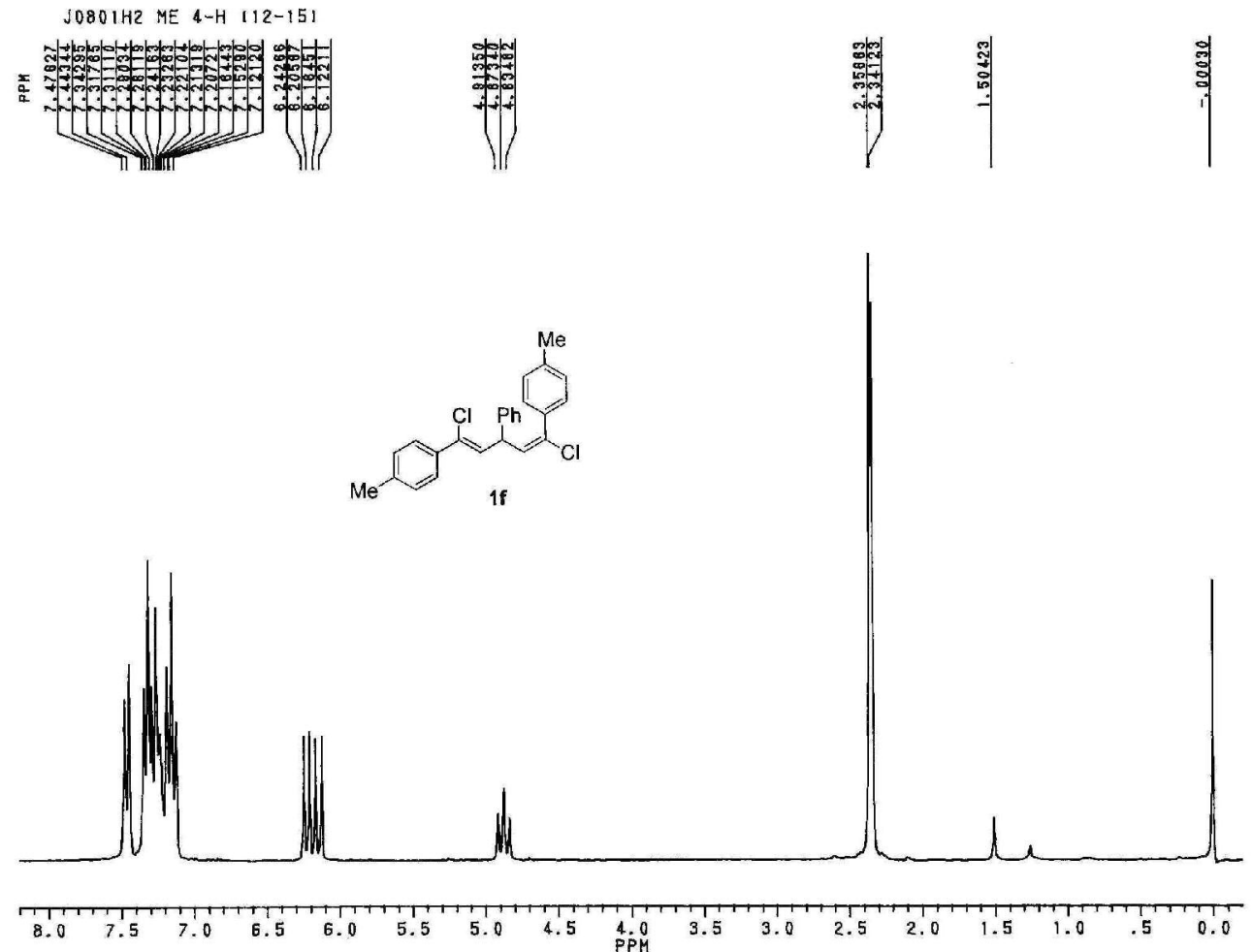


\section{${ }^{13} \mathrm{C}$-NMR spectrum of $1 \mathrm{f}$}

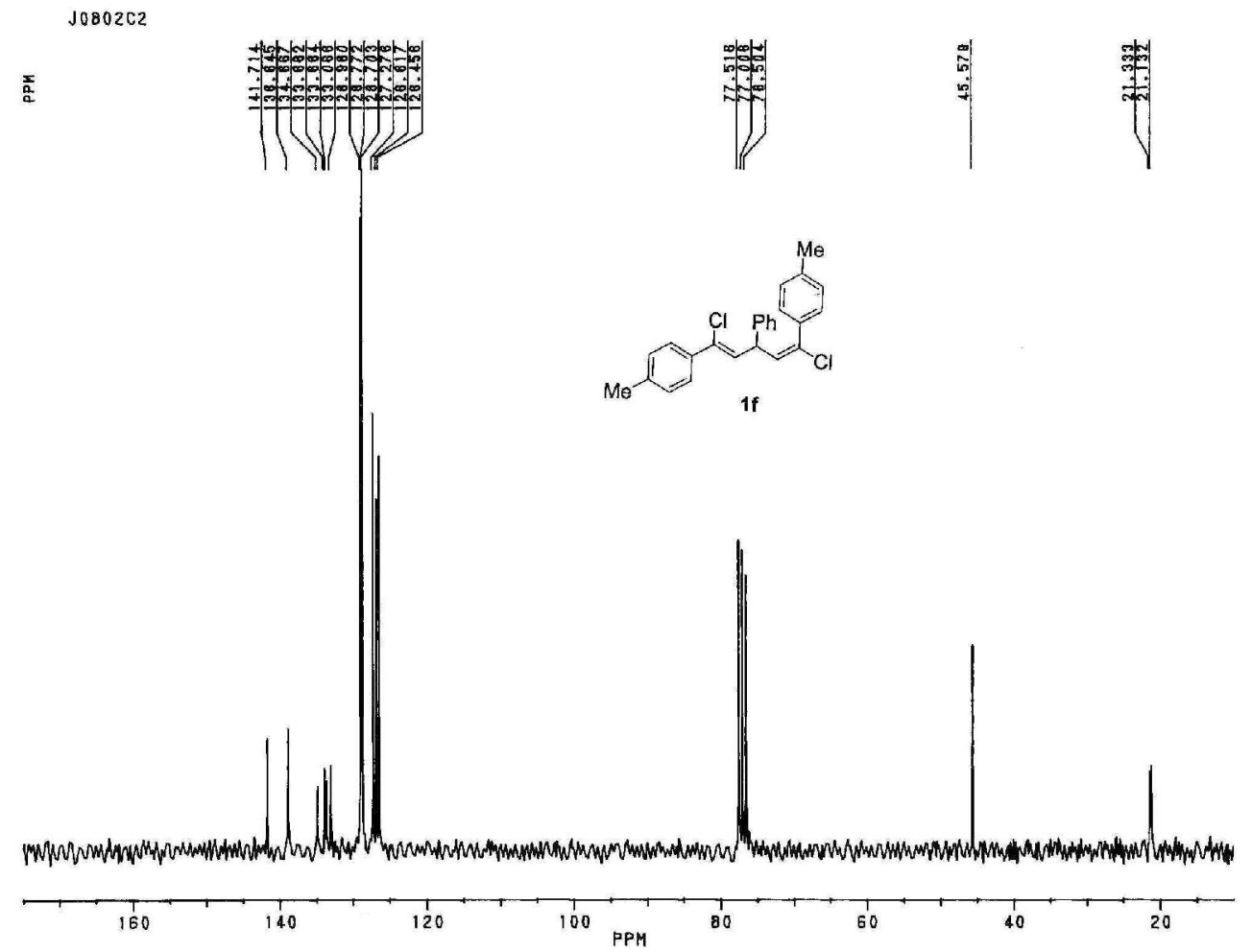




\section{${ }^{1} \mathrm{H}$ NMR spectrum of $1 \mathrm{~g}$}

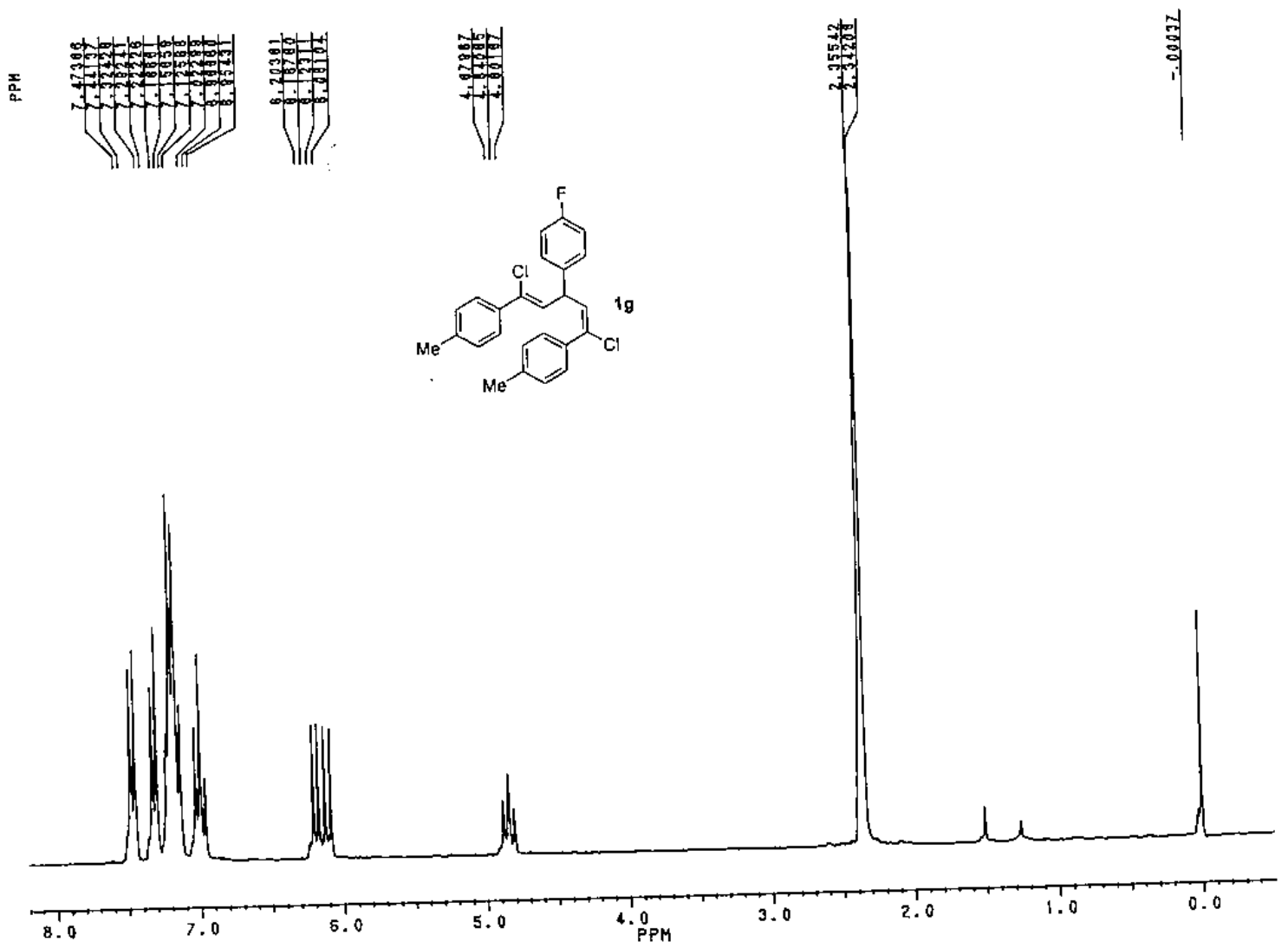




\section{${ }^{13} \mathrm{C}$ - NMR spectrum of $1 \mathrm{~g}$}
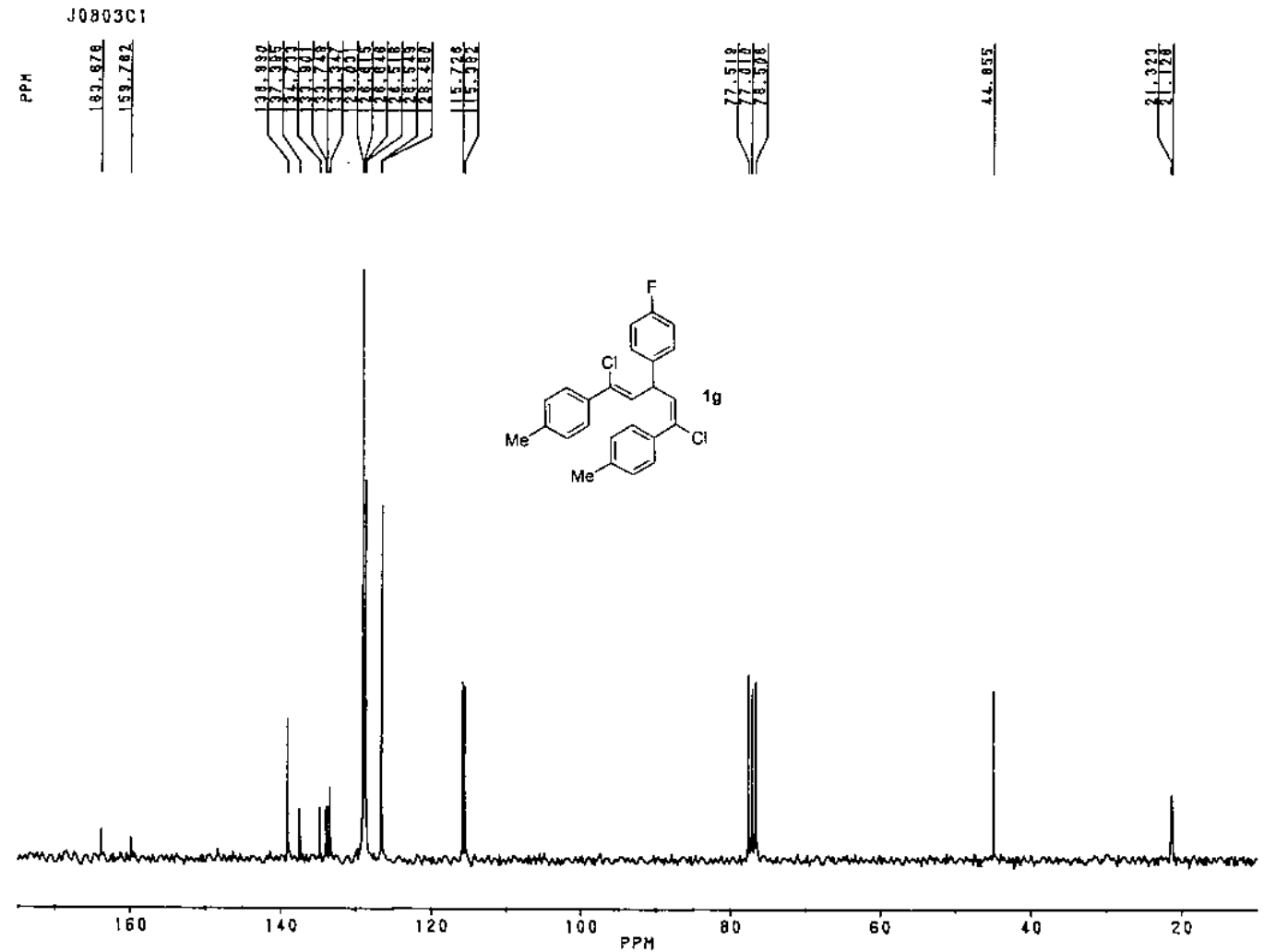


\section{${ }^{1} \mathrm{H}$ - NMR spectrum of $1 \mathrm{~h}$}

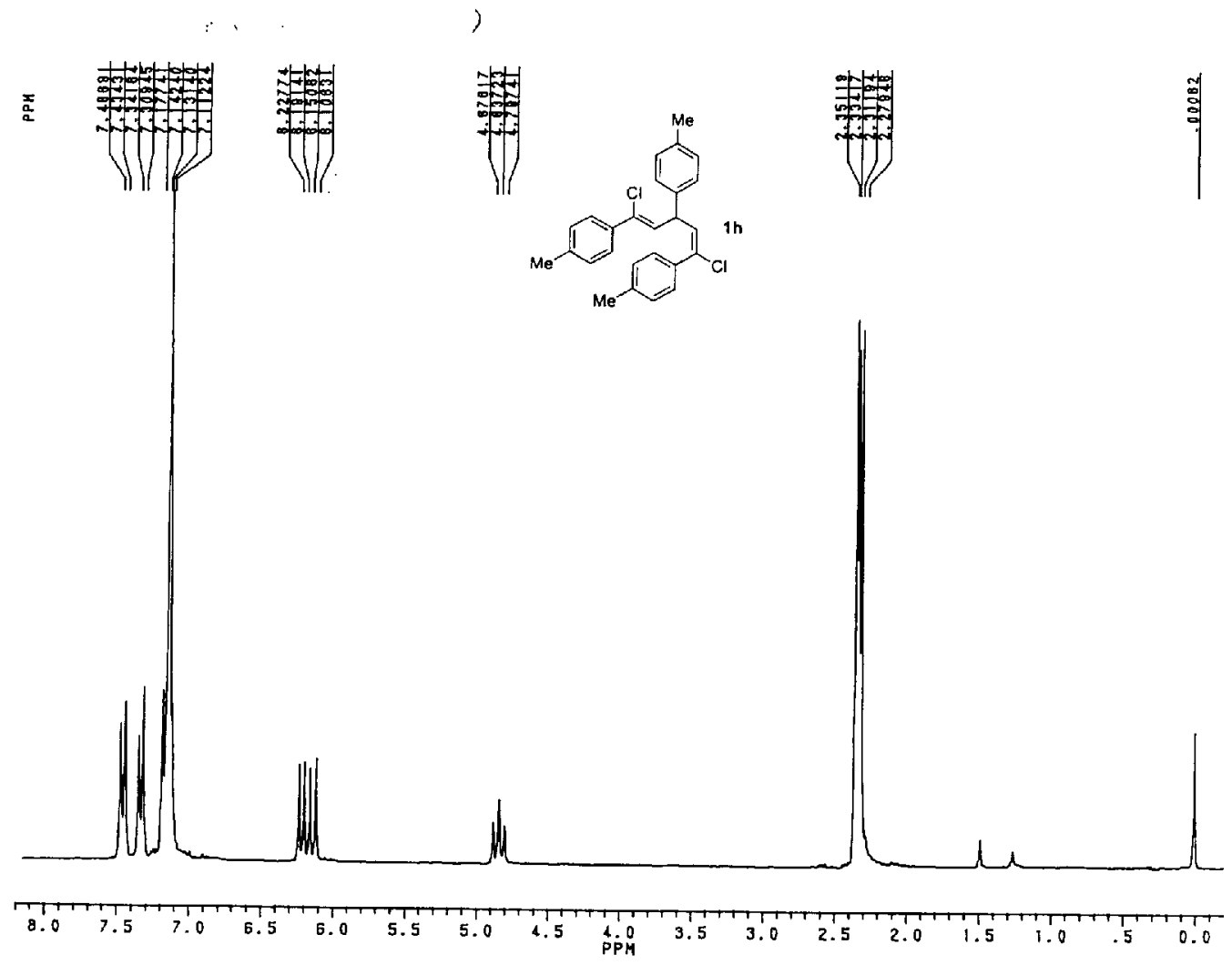




\section{${ }^{13} \mathrm{C}$ - NMR spectrum of $1 \mathrm{~h}$}

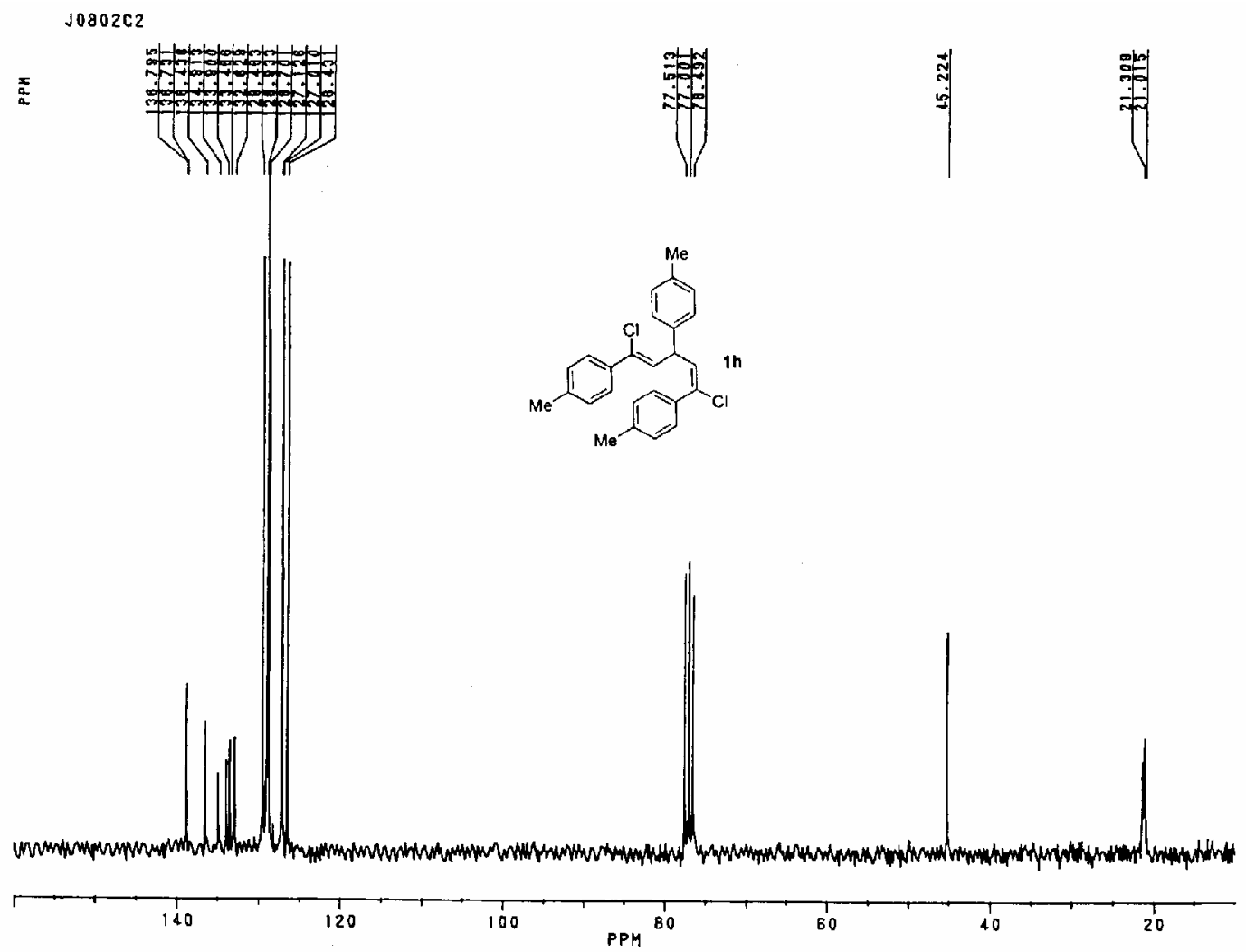




\section{${ }^{1} \mathrm{H}$ - NMR spectrum of $1 \mathrm{i}$}
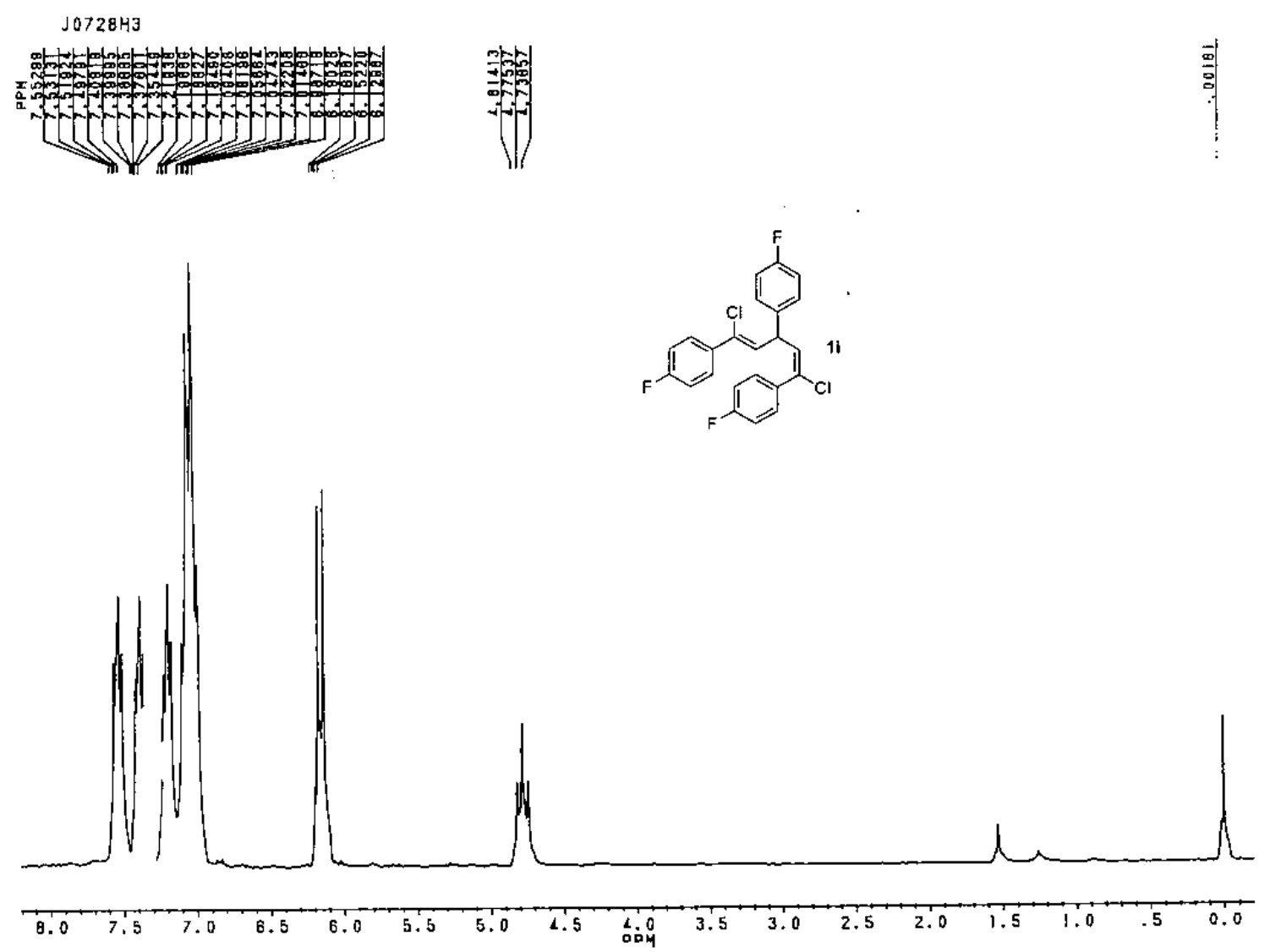


\section{${ }^{13} \mathrm{C}$ - NMR spectrum of $1 \mathrm{i}$}
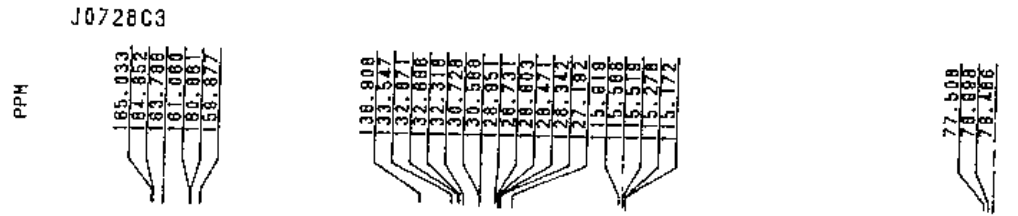

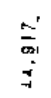

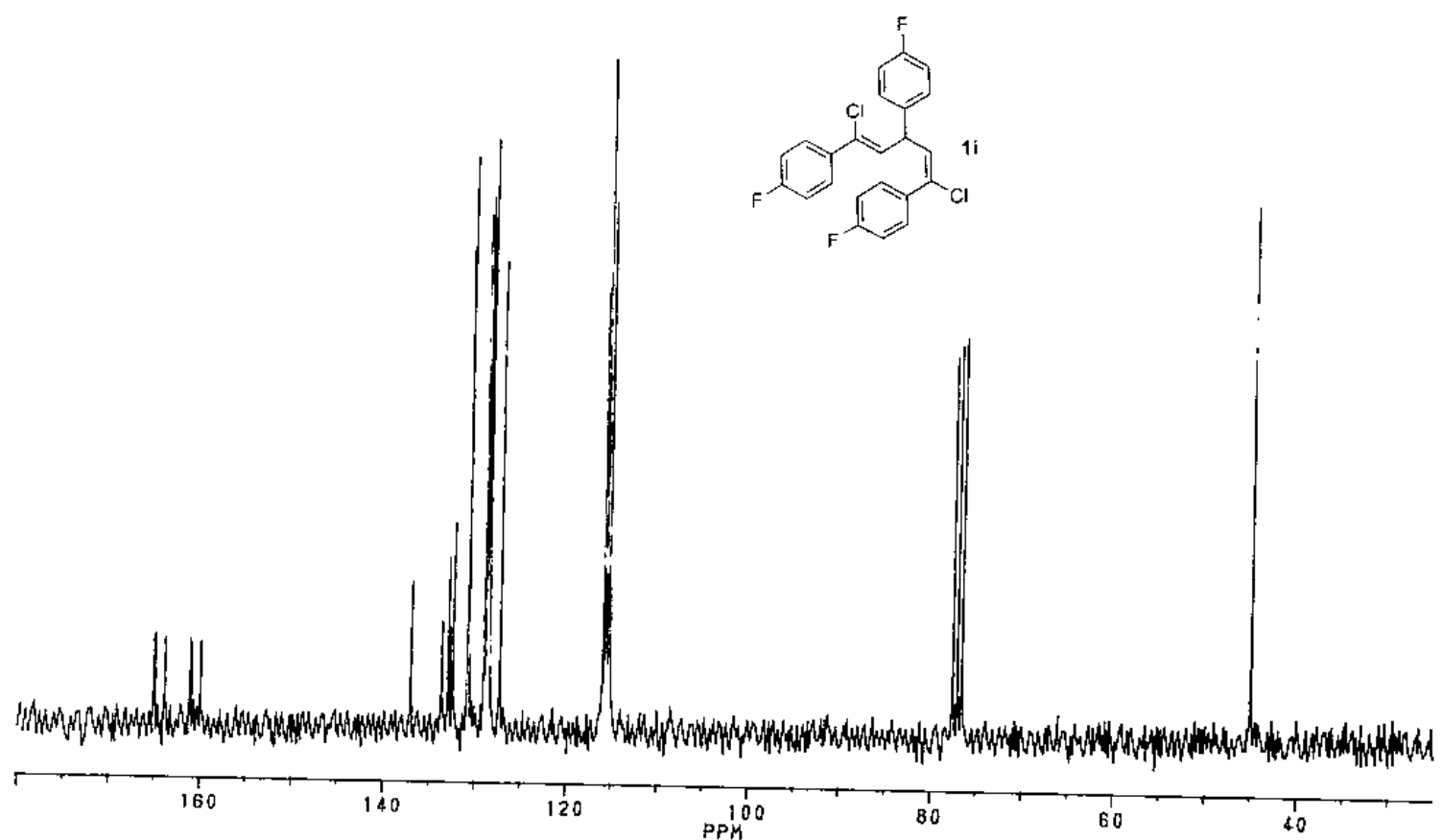




\section{${ }^{1} \mathrm{H}$ - NMR spectrum of $\mathbf{1} \mathbf{j}$}

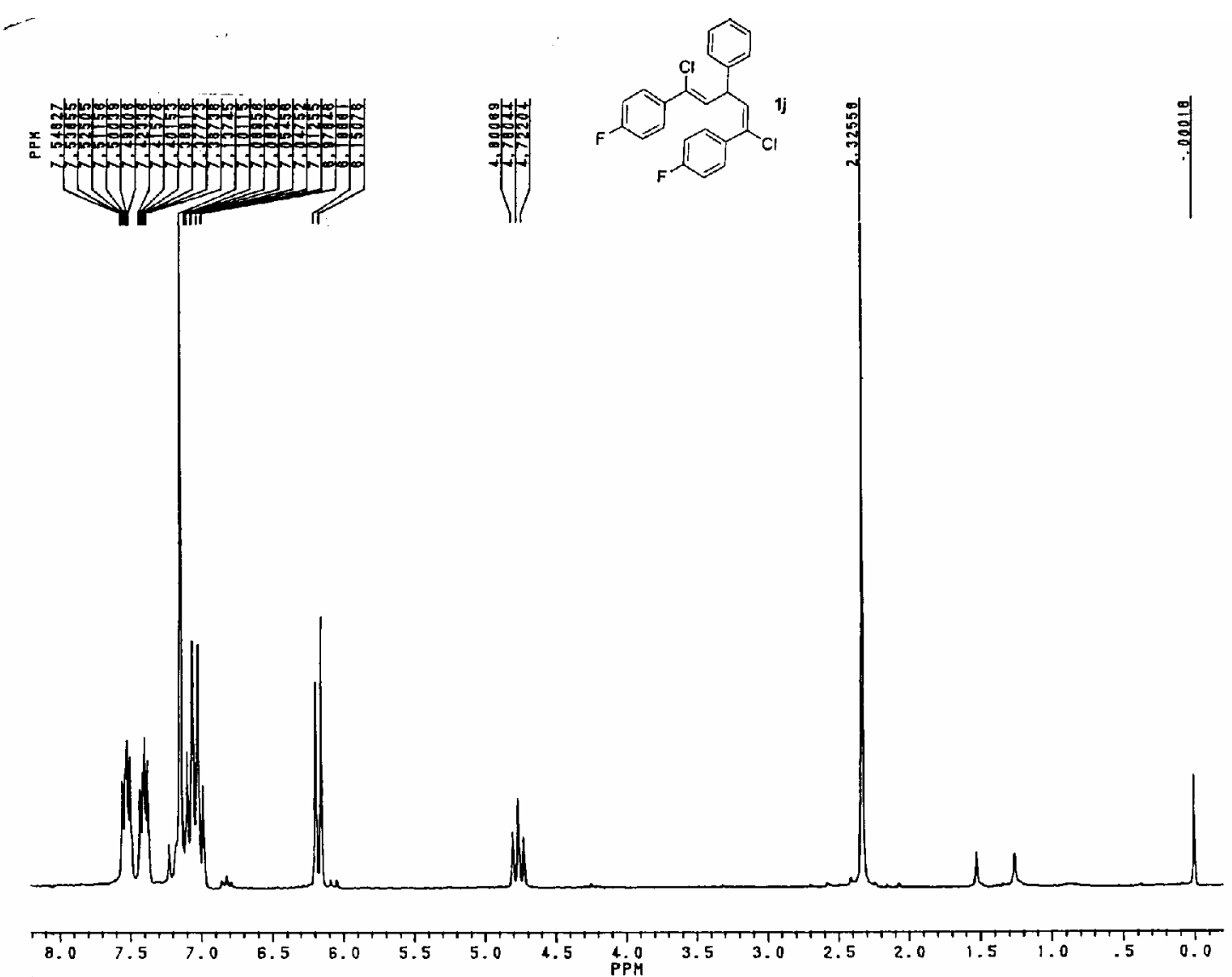




\section{${ }^{13} \mathrm{C}$ - NMR spectrum of $1 \mathbf{j}$}
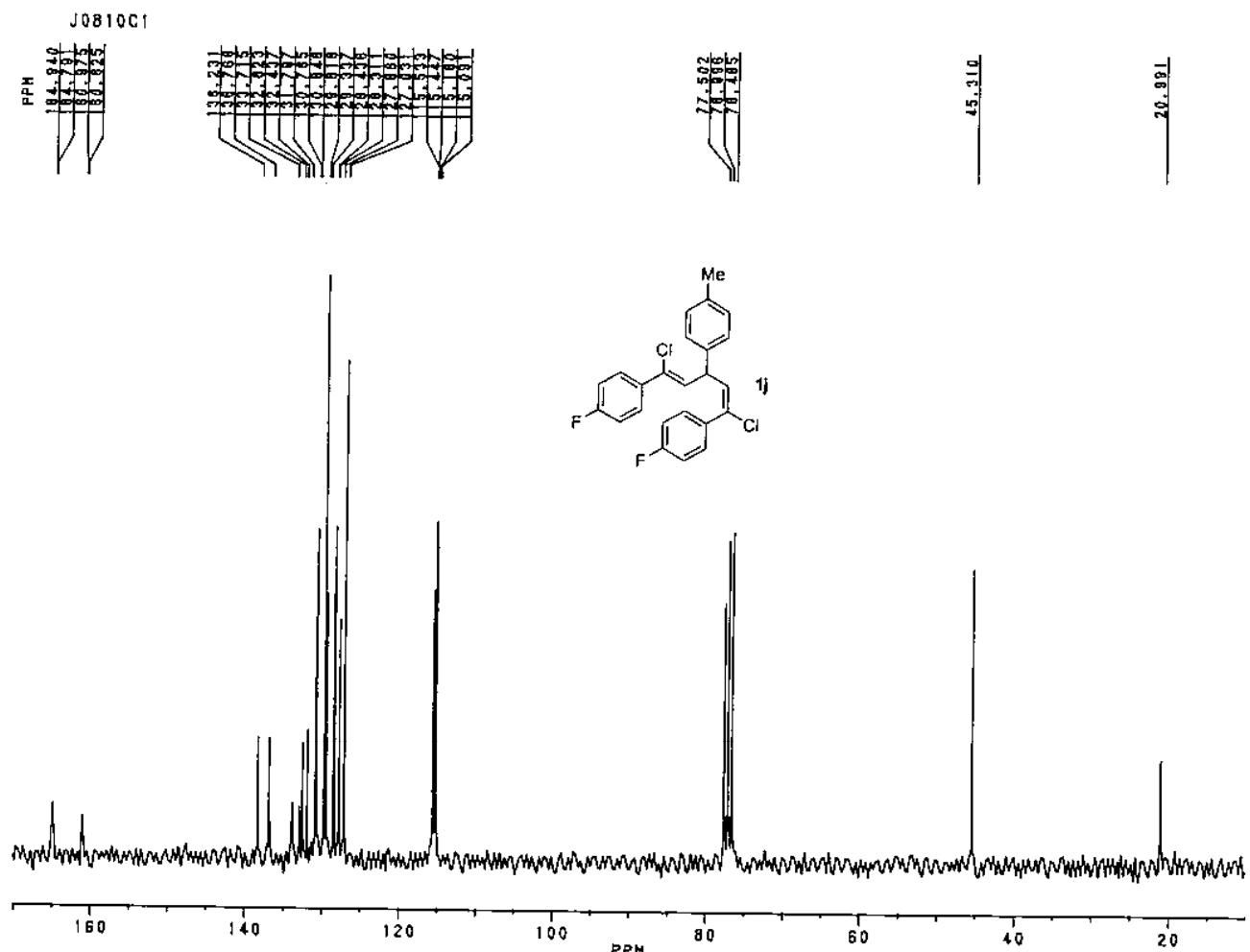


\section{${ }^{1} \mathrm{H}$ - NMR spectrum of $2 \mathrm{a}$}
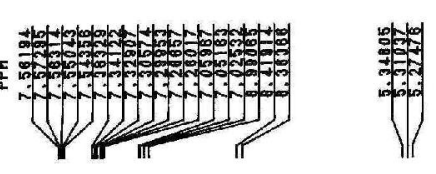

4-FC6H4CHO+2C6H5CCH (BBR3) (FEB 15)

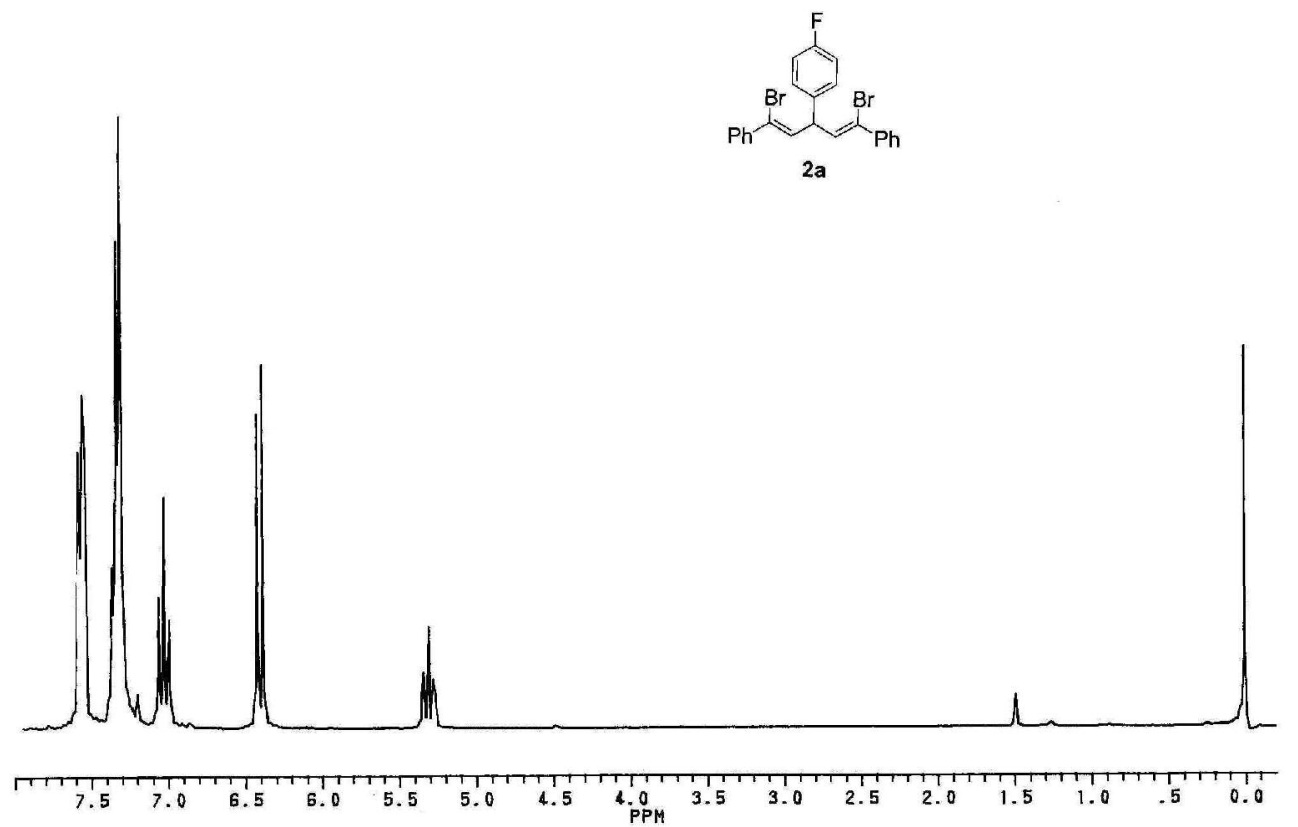




\section{${ }^{13} \mathrm{C}-\mathrm{NMR}$ spectrum of $2 \mathrm{a}$}
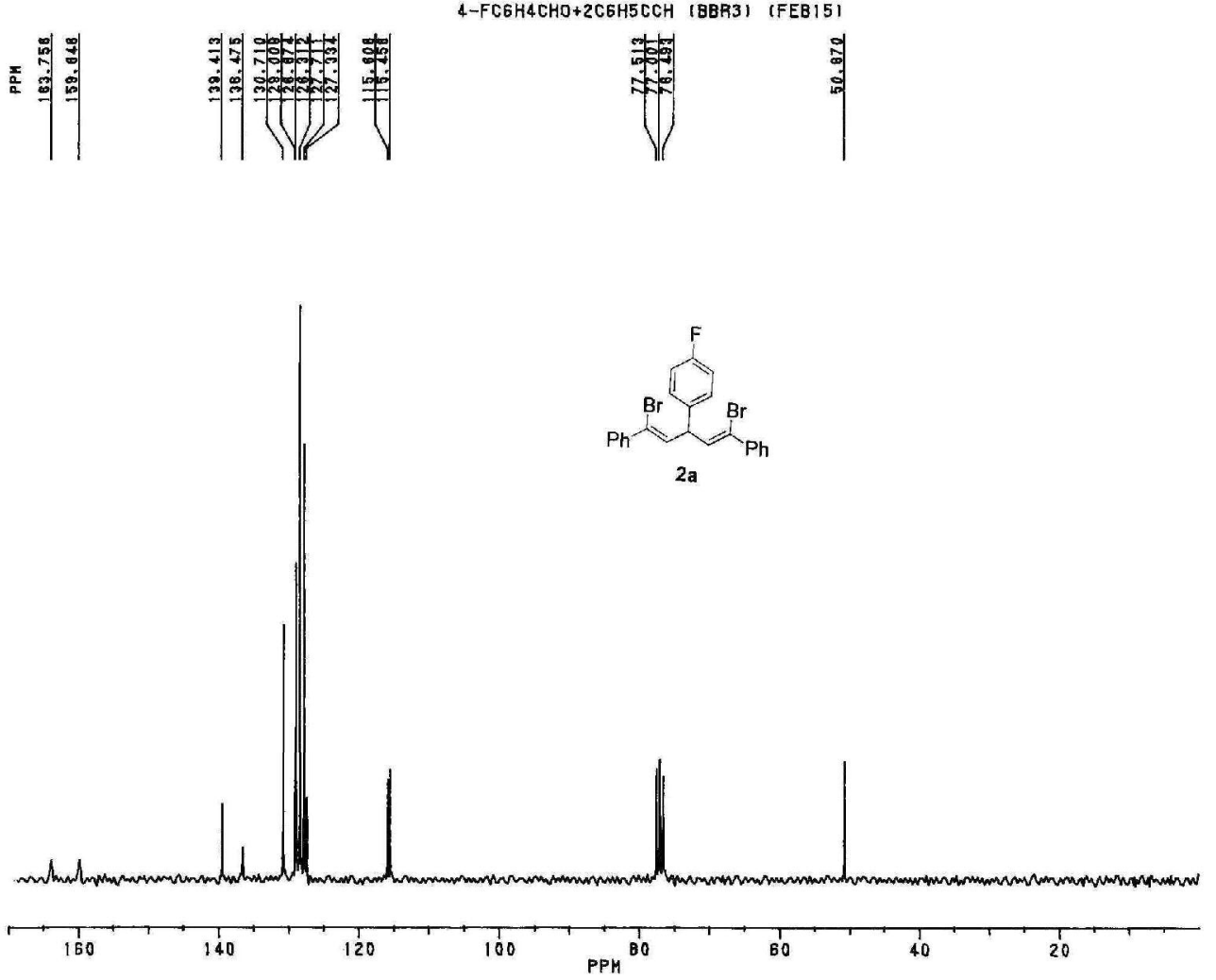


\section{${ }^{1} \mathrm{H}$ - NMR spectrum of $2 b$}

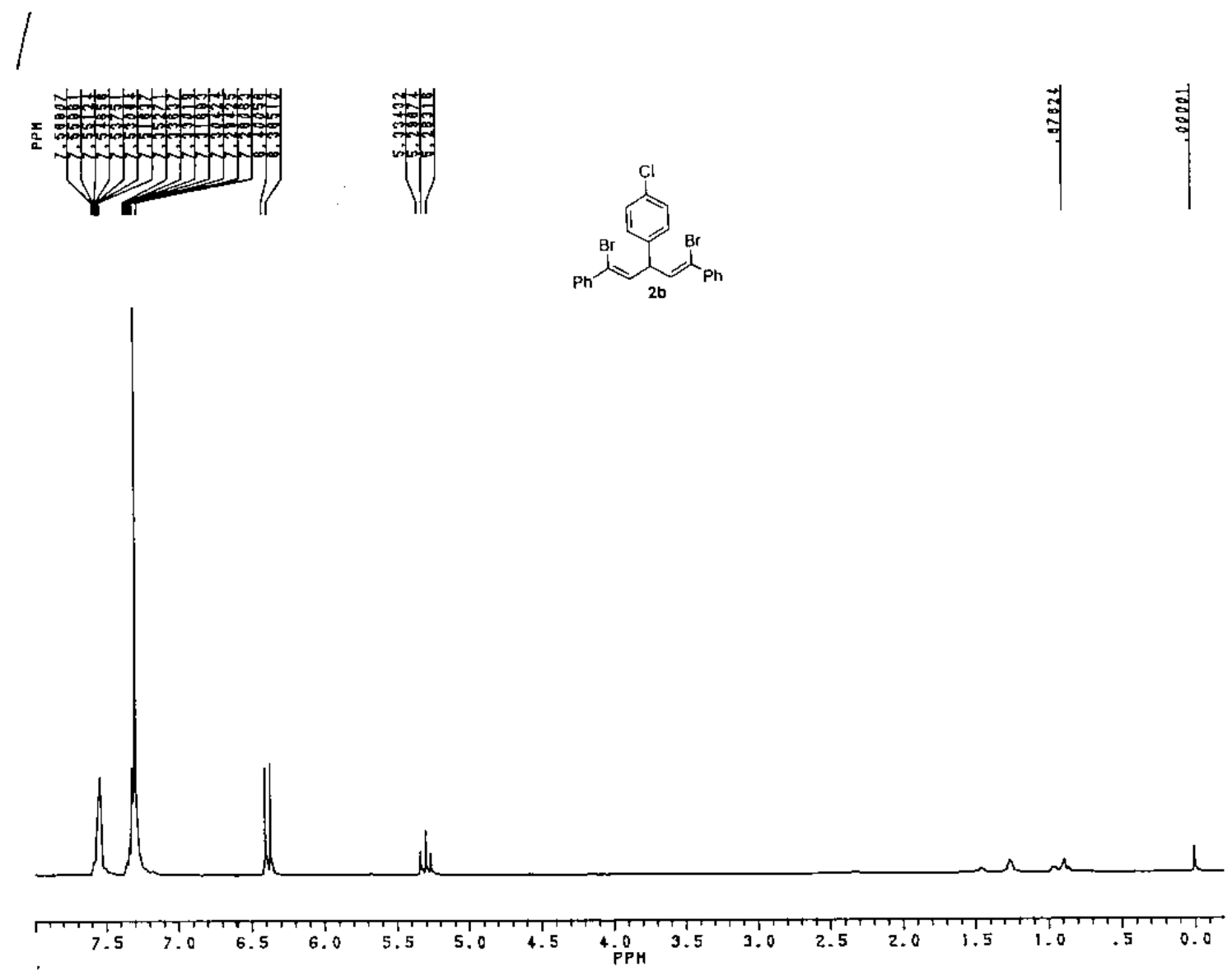




\section{${ }^{13} \mathrm{C}$ - NMR spectrum of $2 \mathrm{~b}$}
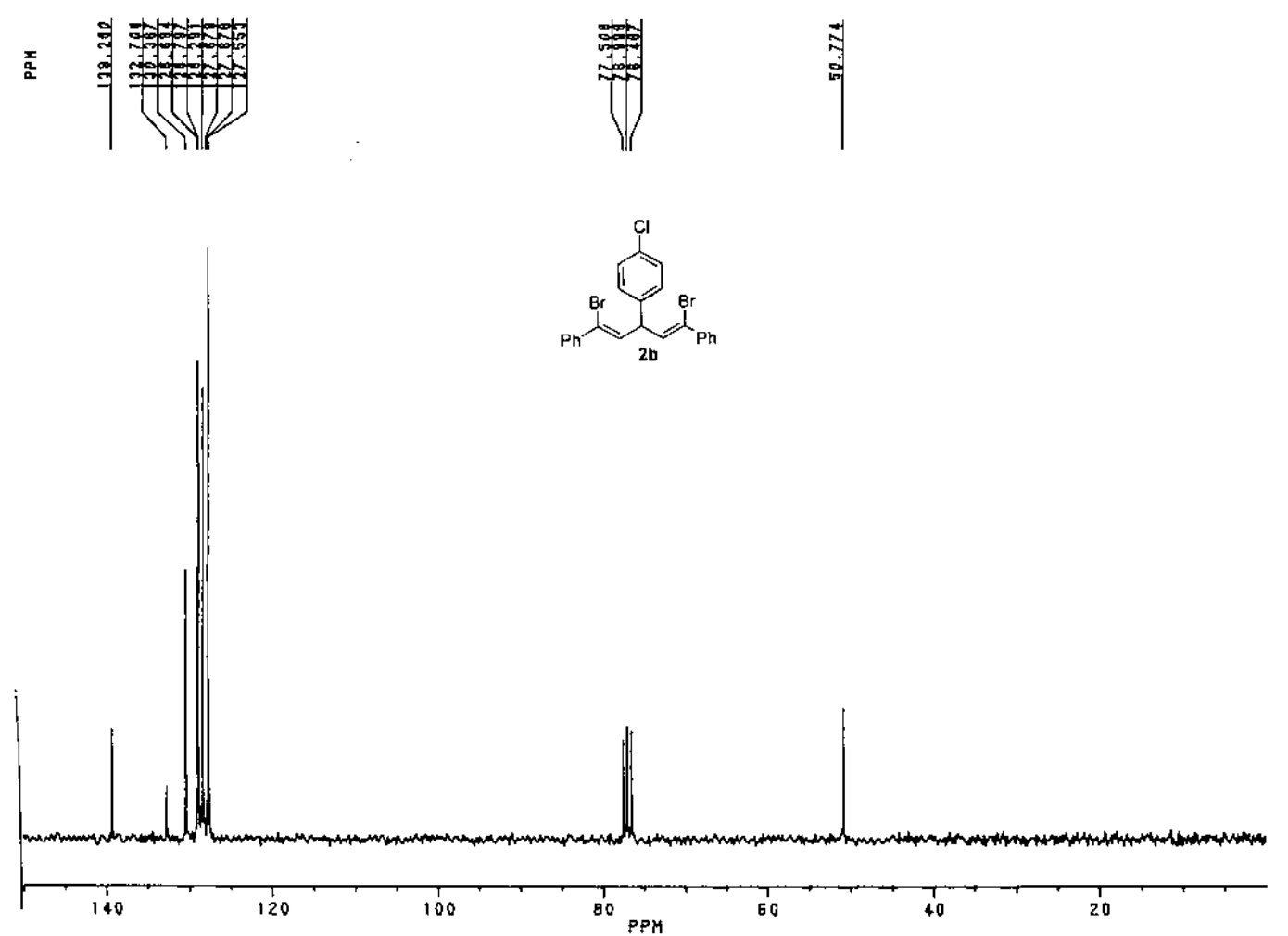


\section{${ }^{1} \mathrm{H}$ - NMR spectrum of $2 \mathrm{c}$}
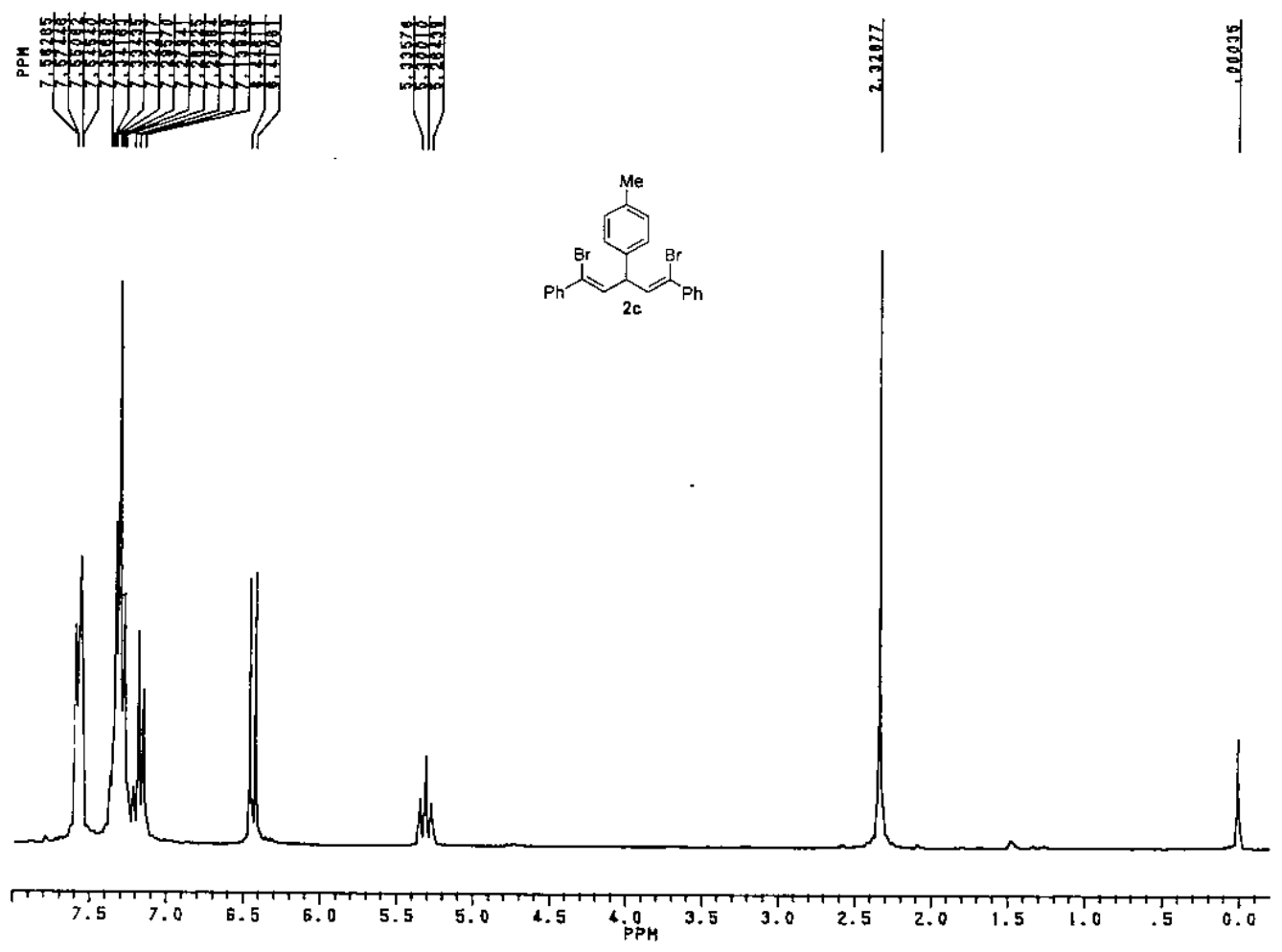


\section{${ }^{13} \mathrm{C}$ - NMR spectrum of $2 \mathrm{c}$}

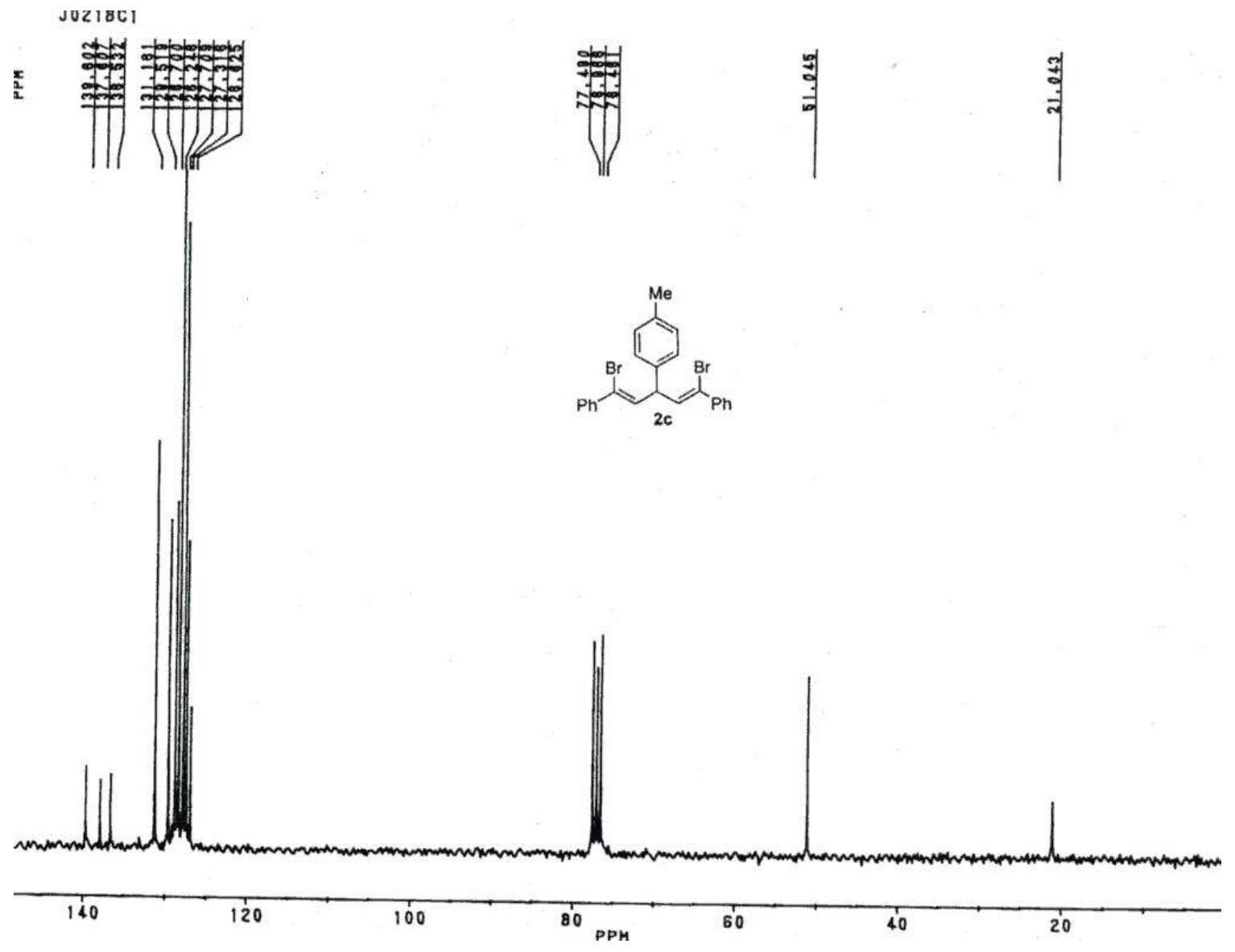




\section{${ }^{1} \mathrm{H}$ - NMR spectrum of $2 d$}
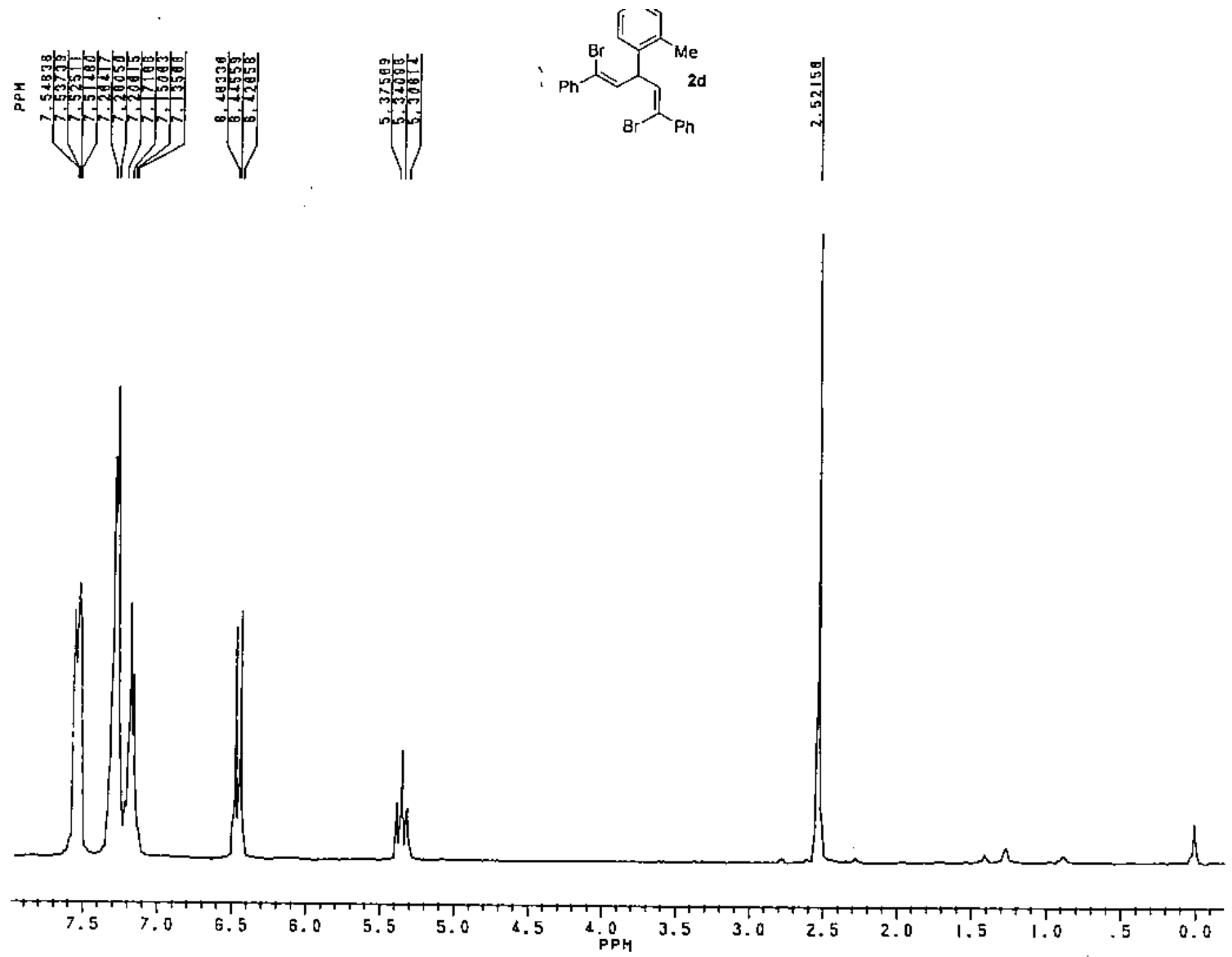


\section{${ }^{13} \mathrm{C}$ - NMR spectrum of $2 \mathrm{~d}$}

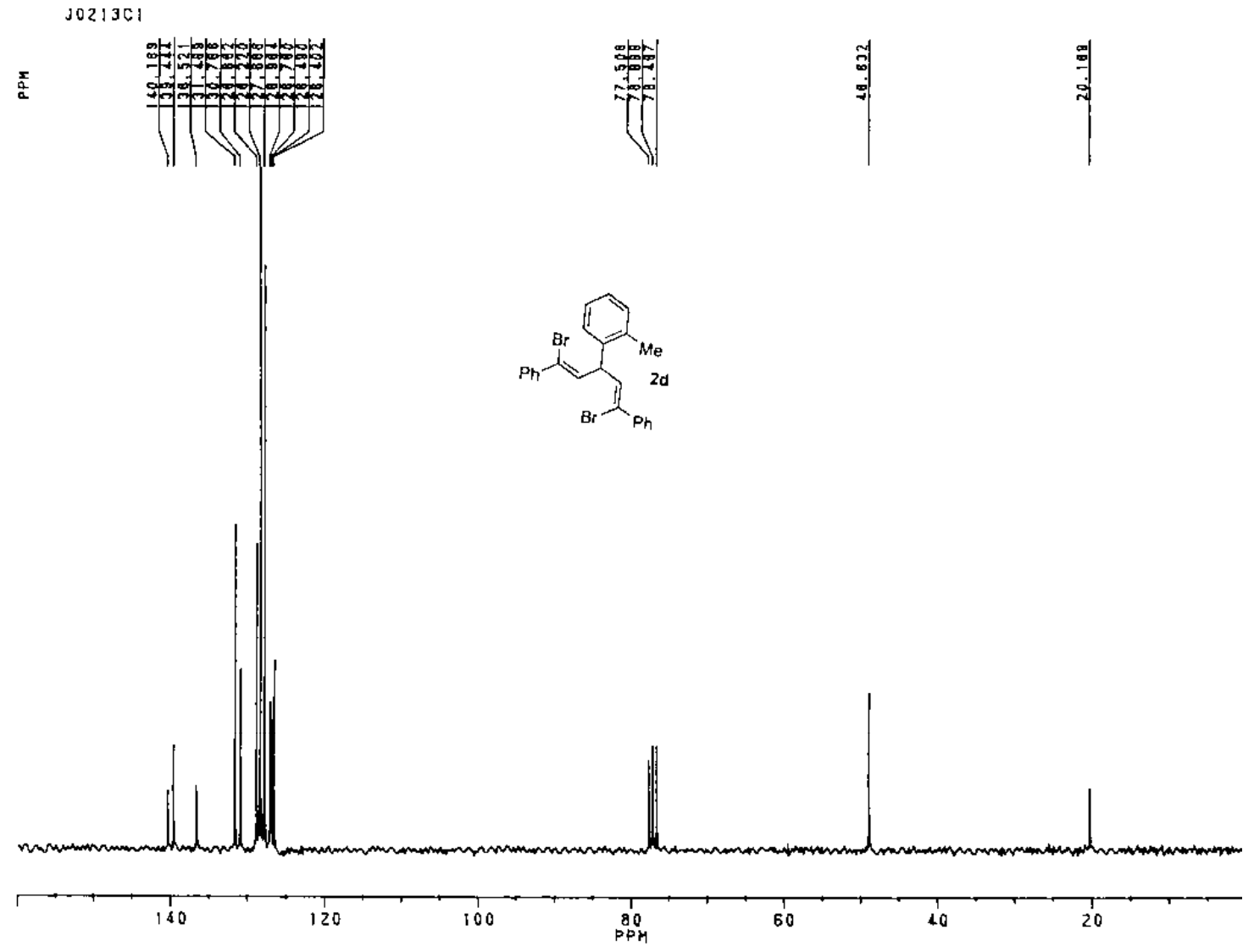




\section{${ }^{1} \mathrm{H}$ - NMR spectrum of $2 \mathrm{e}$}

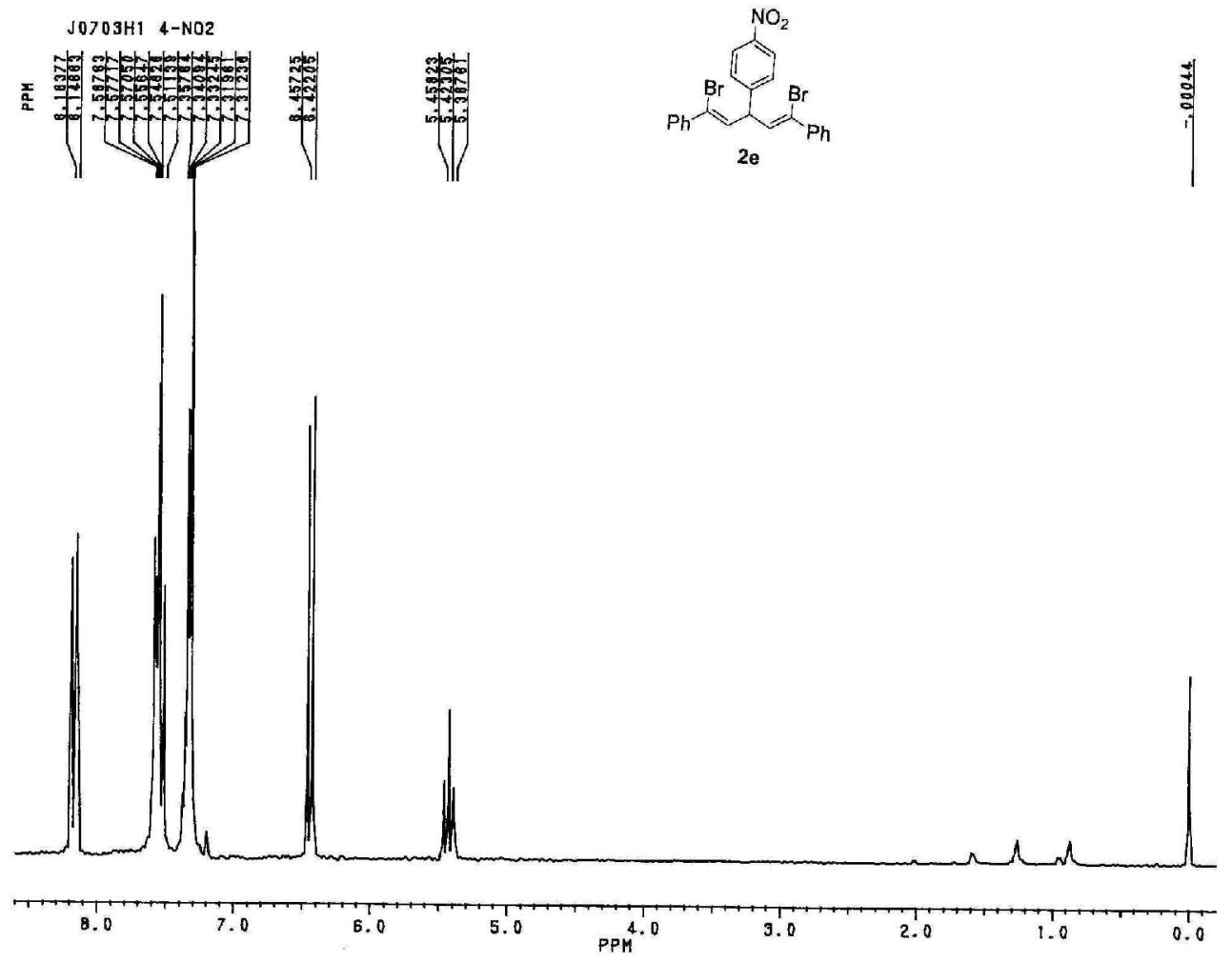




\section{${ }^{13} \mathrm{C}$-NMR spectrum of $2 \mathrm{e}$}

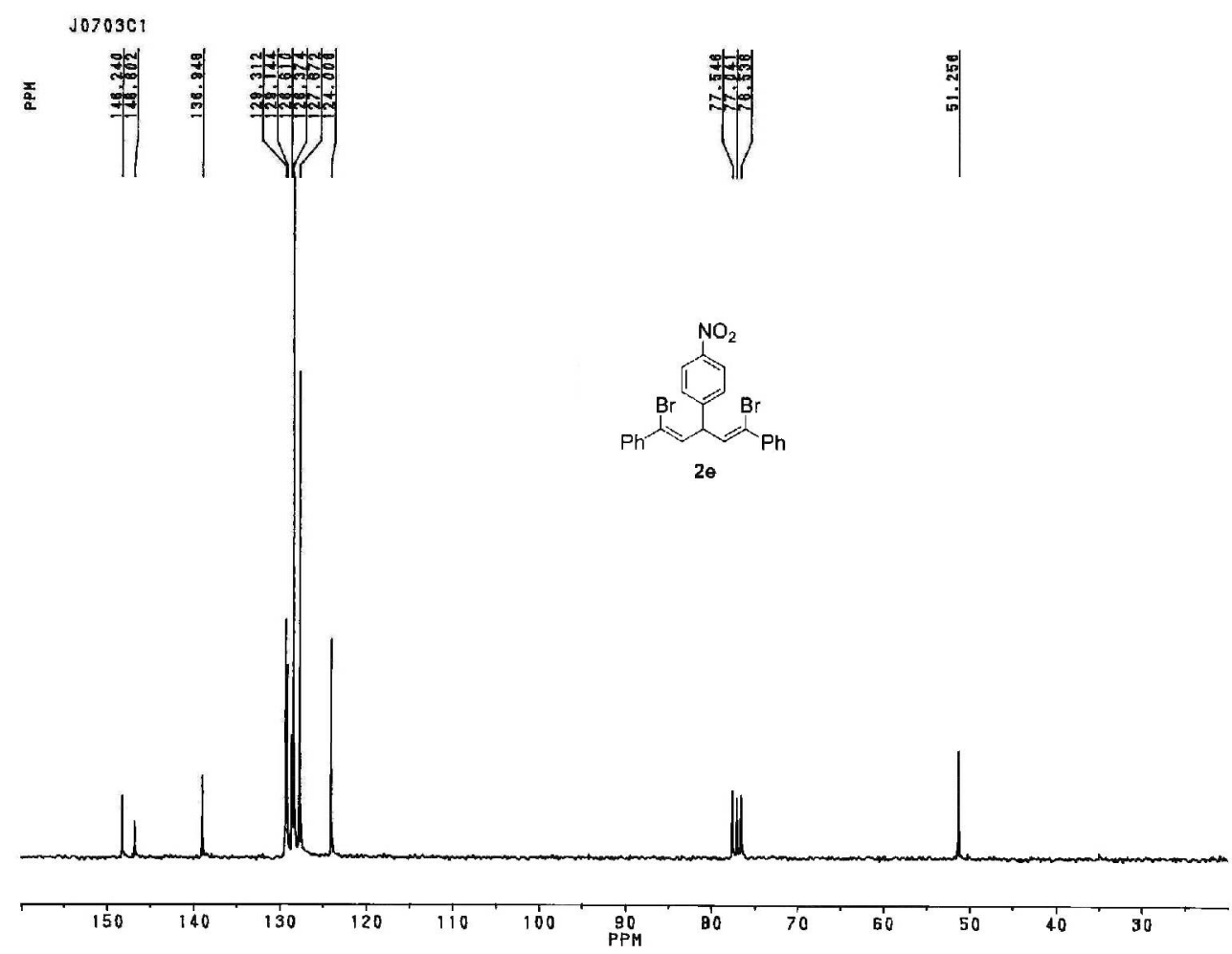




\section{${ }^{1} \mathrm{H}$ - NMR spectrum of $2 \mathrm{f}$}
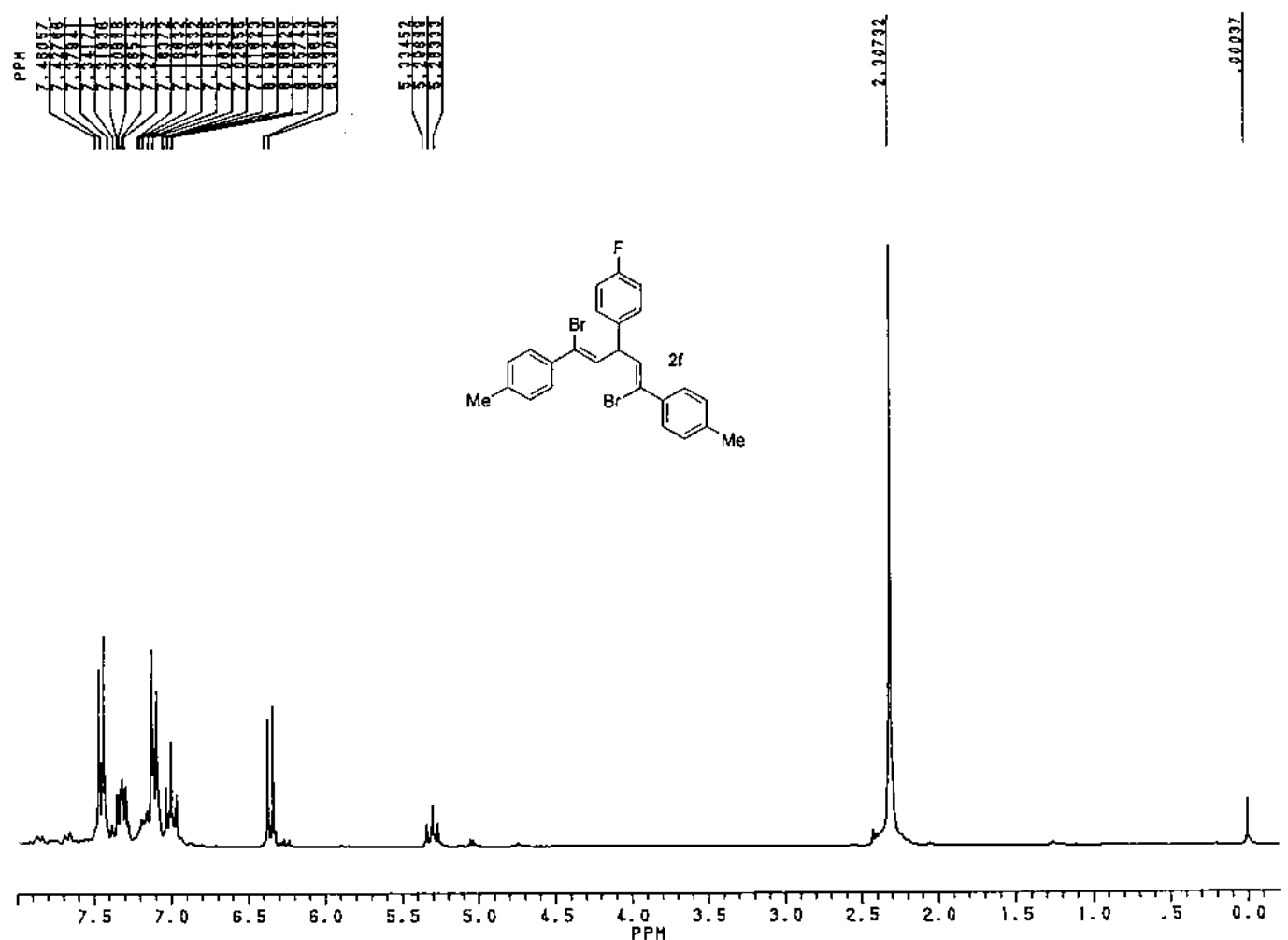
${ }^{13} \mathrm{C}$ - NMR spectrum of $2 \mathrm{f}$

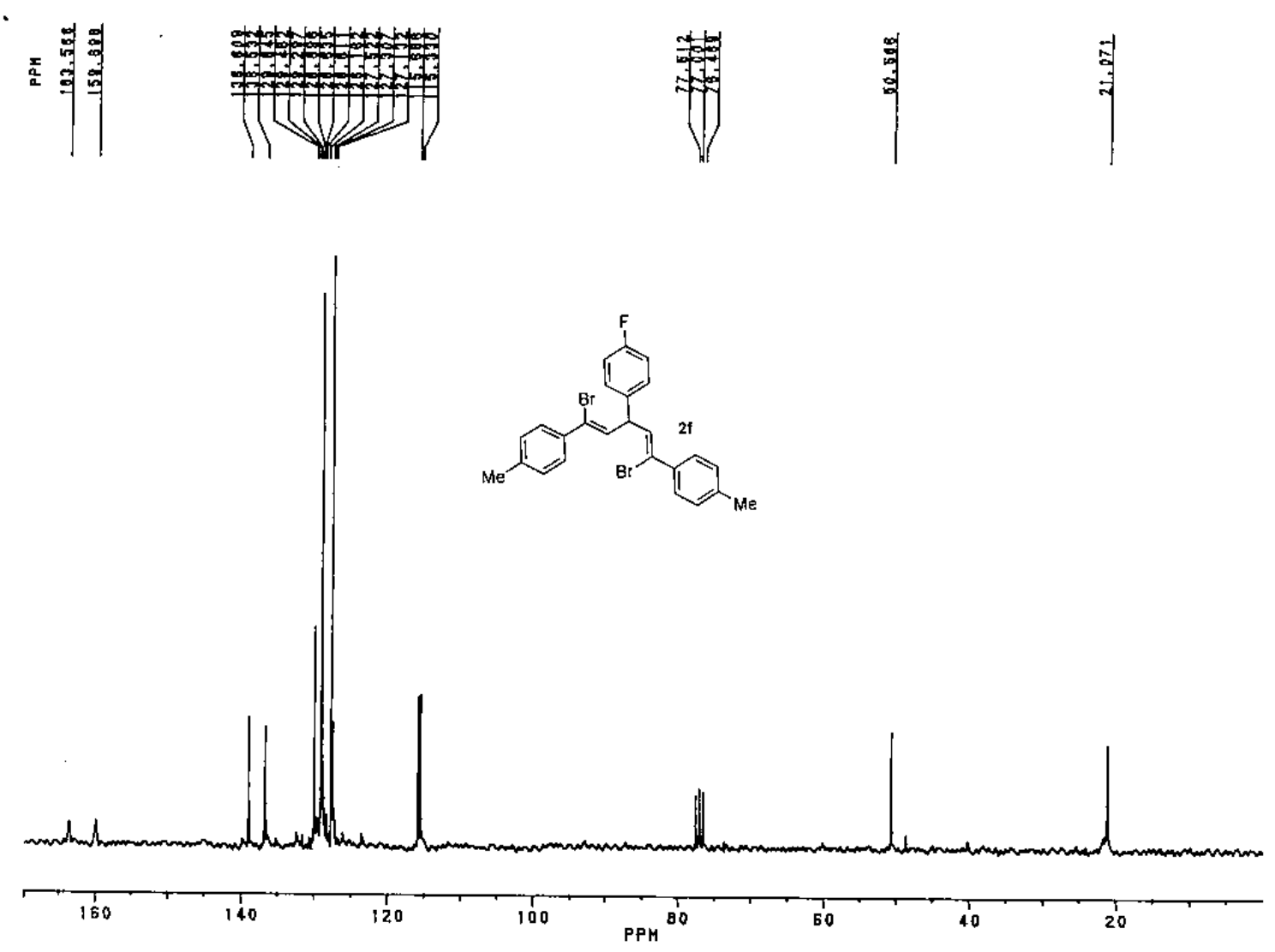




\section{${ }^{1} \mathrm{H}$ - NMR spectrum of $\mathbf{2 g}$}
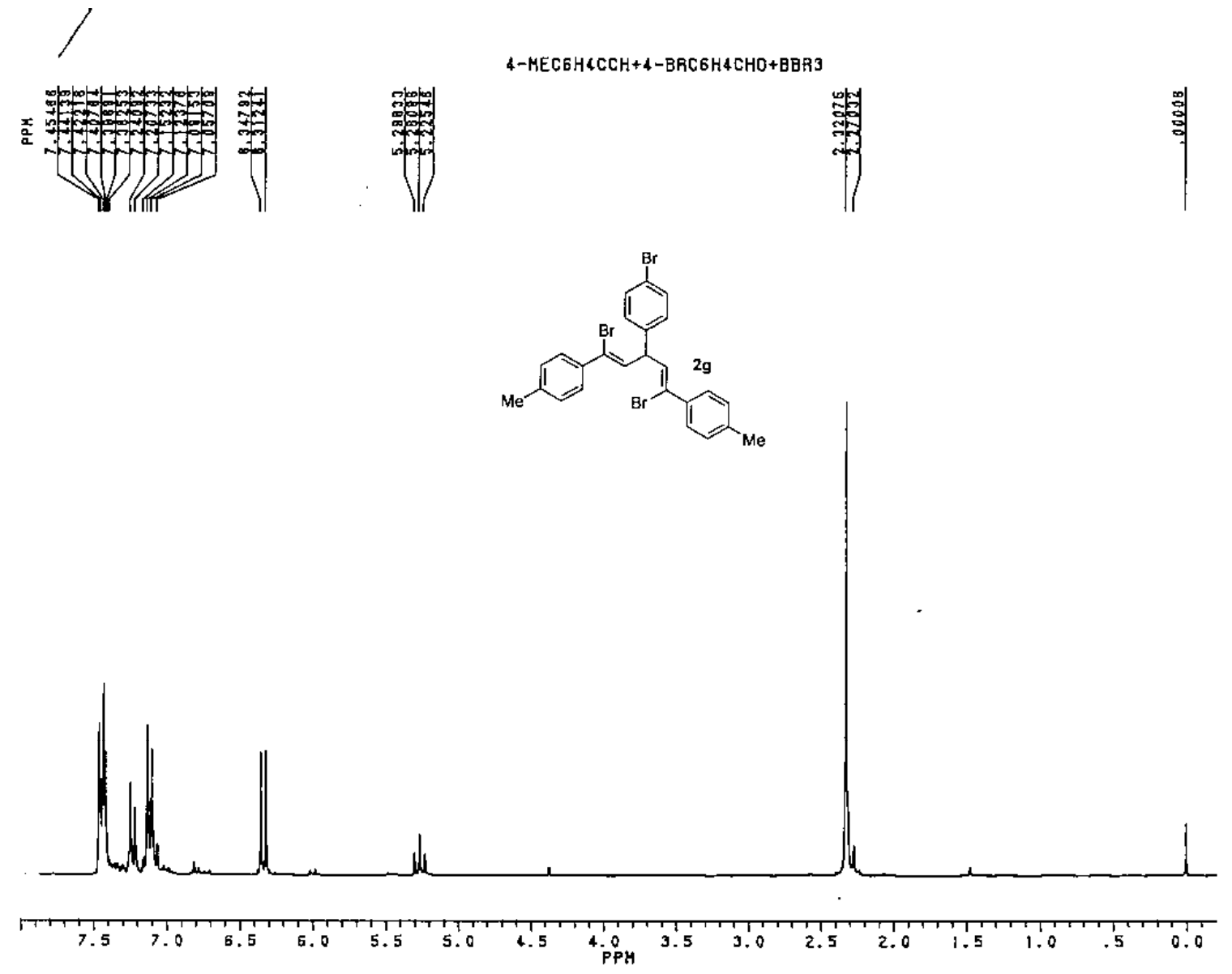


\section{${ }^{13} \mathrm{C}$ - NMR spectrum of $2 \mathrm{~g}$}
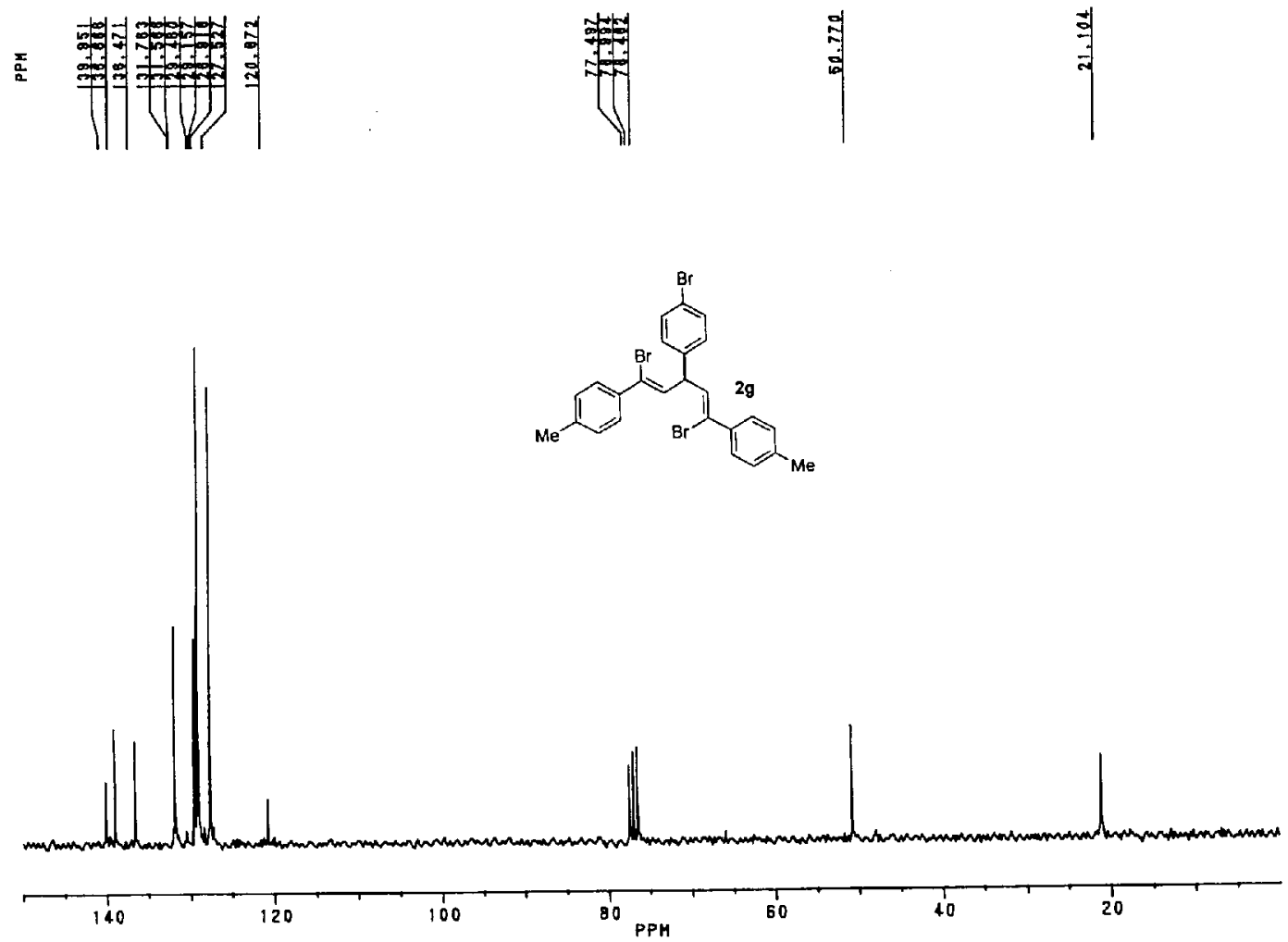


\section{${ }^{1} \mathrm{H}$ - NMR spectrum of $2 \mathrm{~h}$}
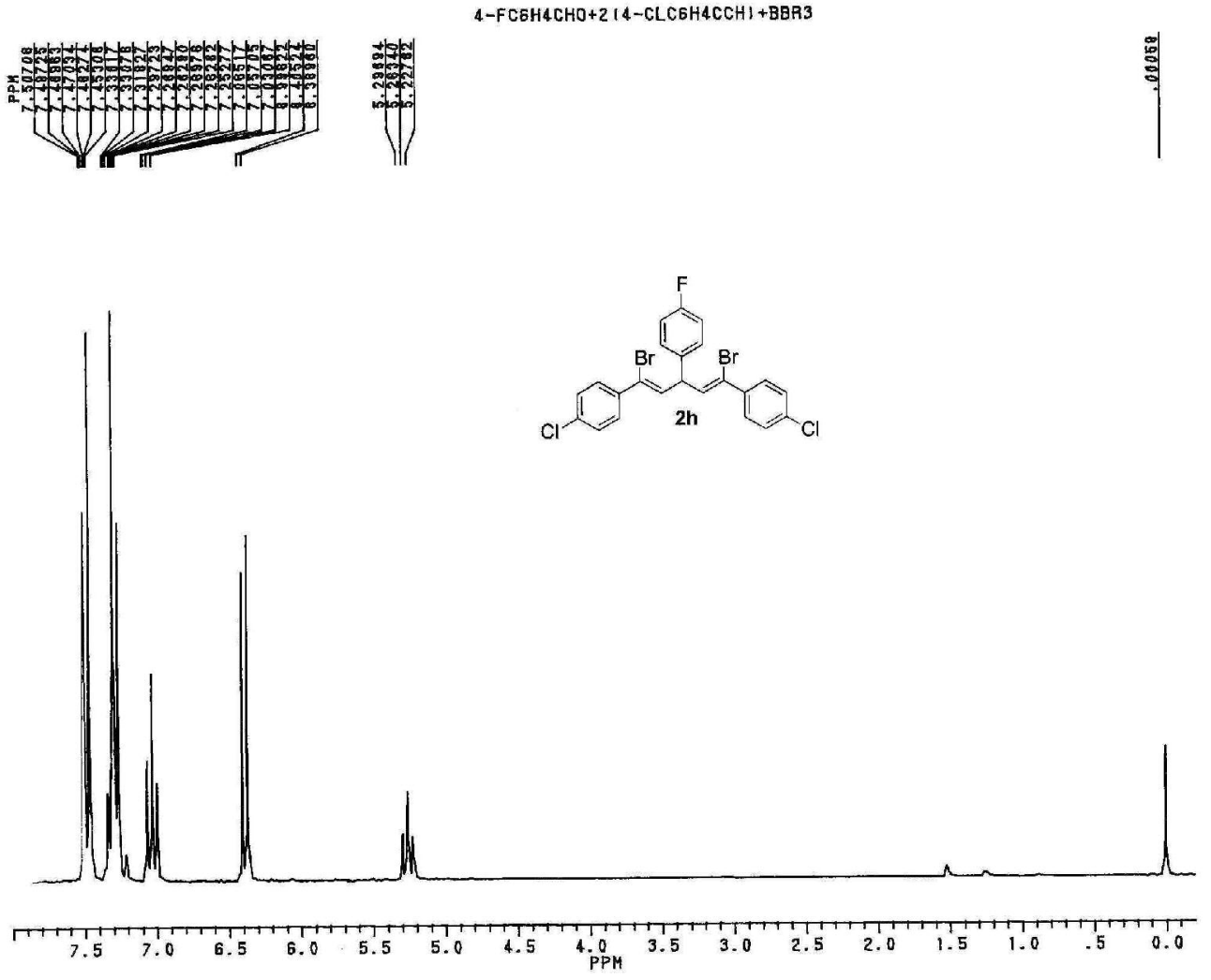


\section{${ }^{13} \mathrm{C}-\mathrm{NMR}$ spectrum of $2 \mathrm{~h}$}

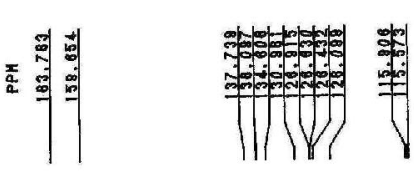

JW0614C
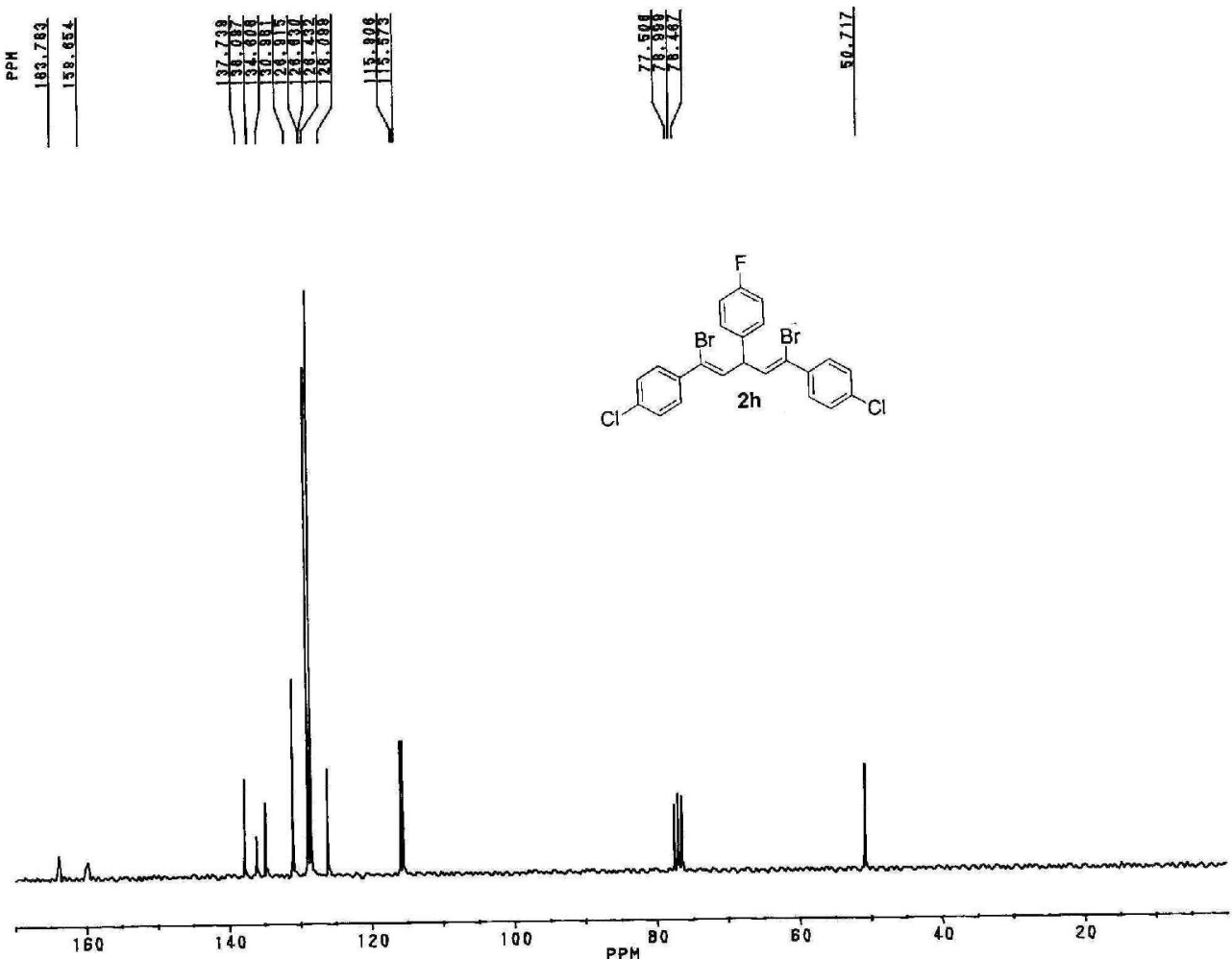


\section{${ }^{1} \mathrm{H}$ - NMR spectrum of $2 \mathrm{i}$}

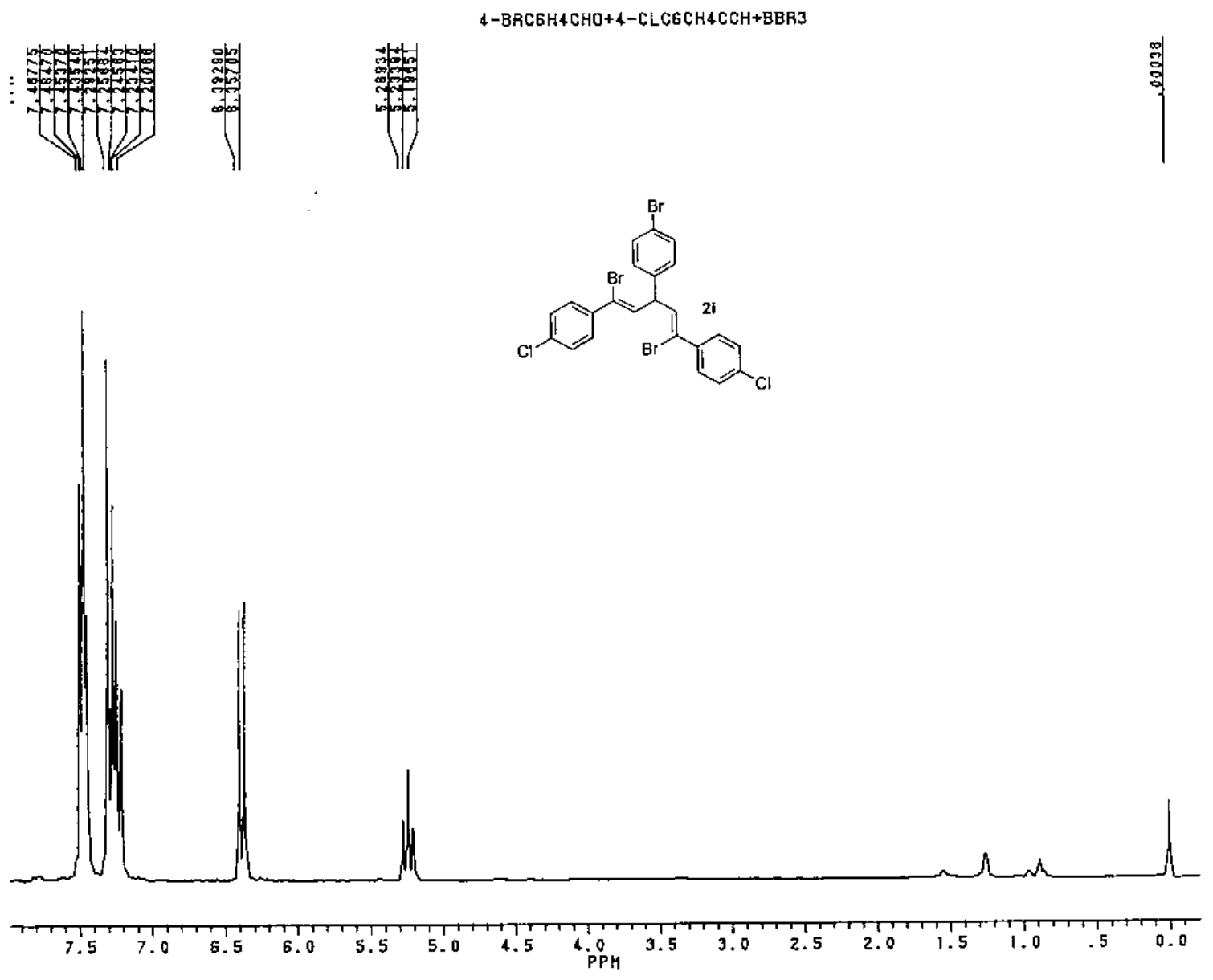




\section{${ }^{13} \mathrm{C}$ - NMR spectrum of $2 \mathrm{i}$}
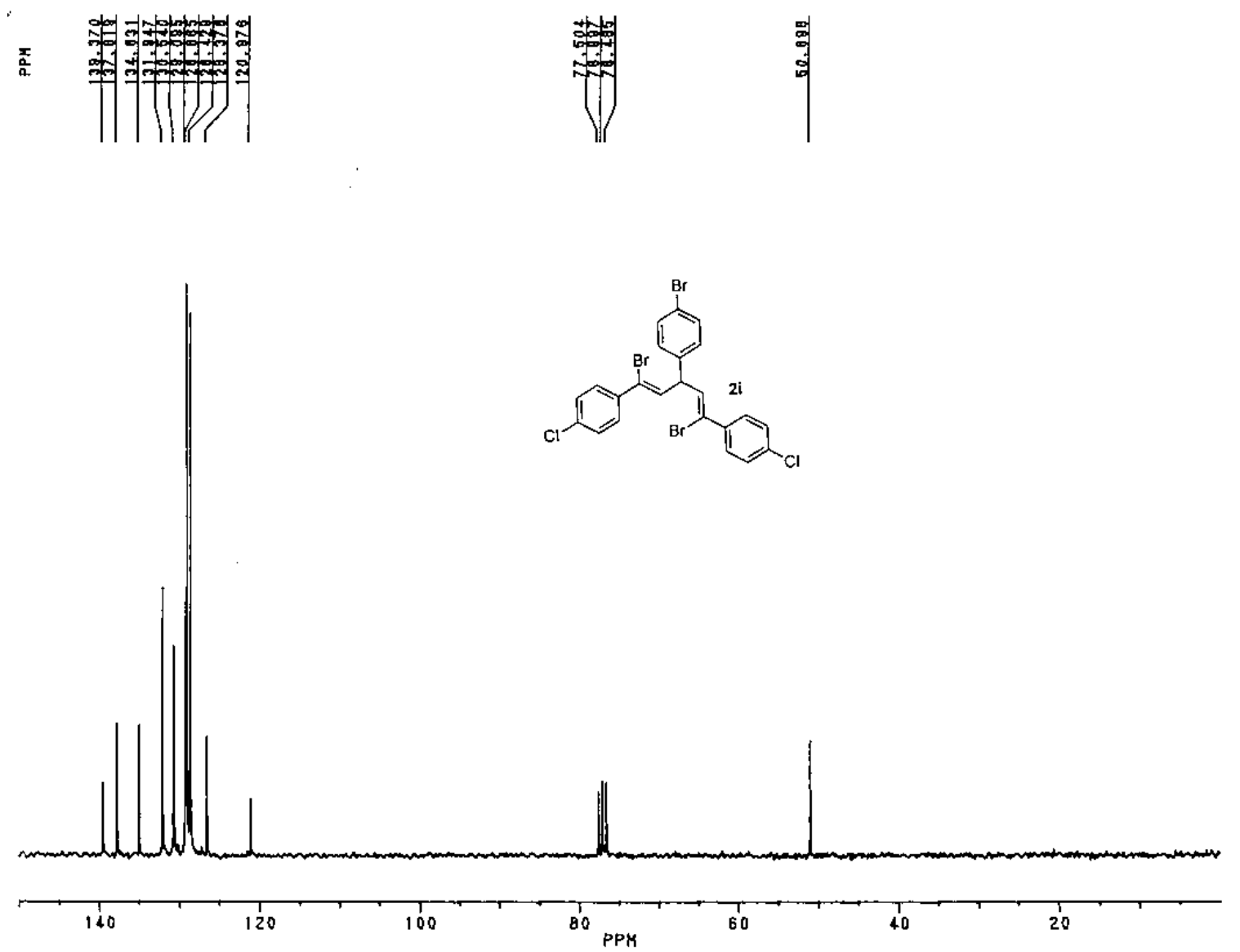


\section{${ }^{1} \mathrm{H}$ - NMR spectrum of $2 \mathrm{j}$}

/
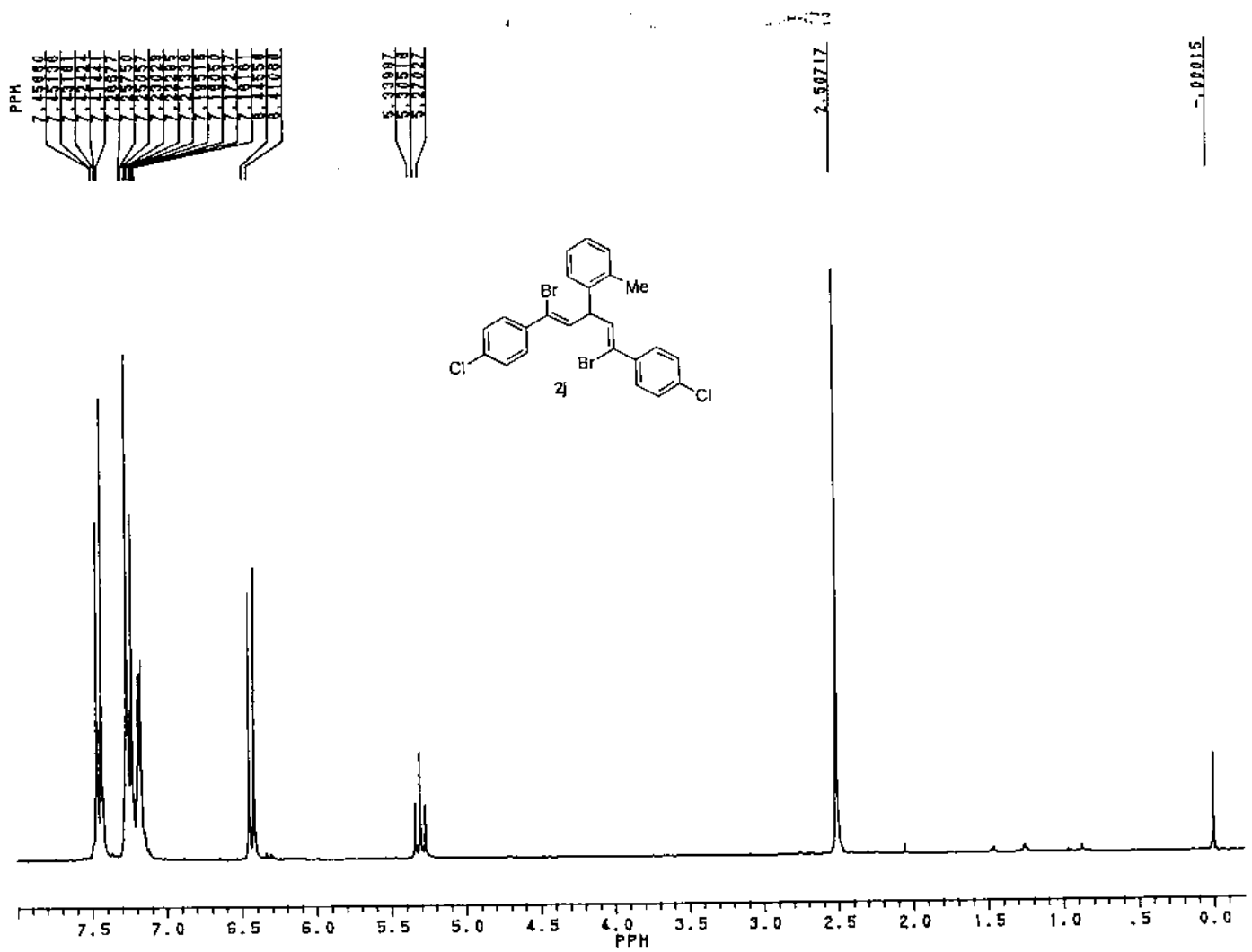


\section{${ }^{13} \mathrm{C}$ - NMR spectrum of $2 \mathrm{j}$}
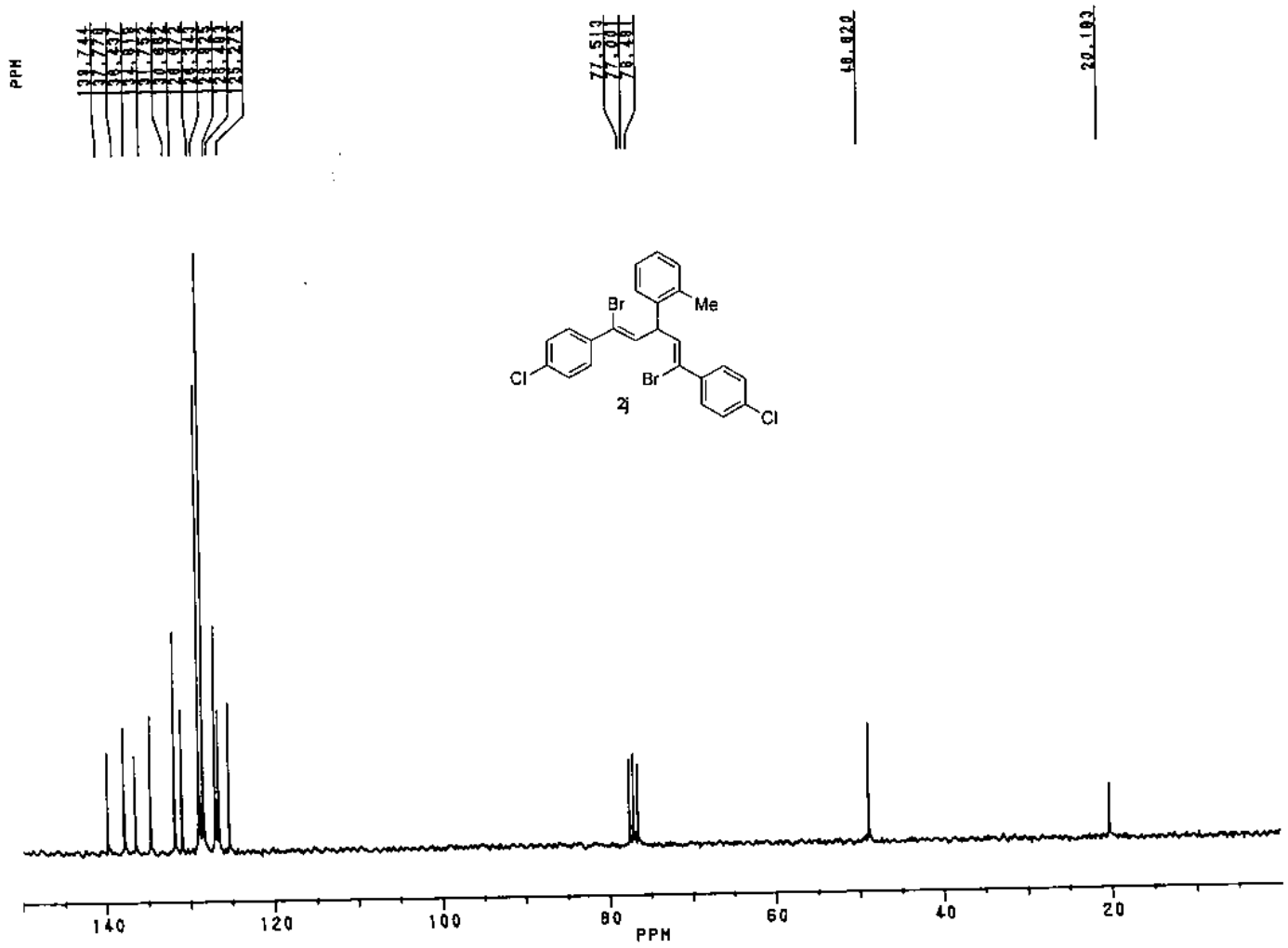


\section{${ }^{1}$ H- NMR spectrum of $8 a$}
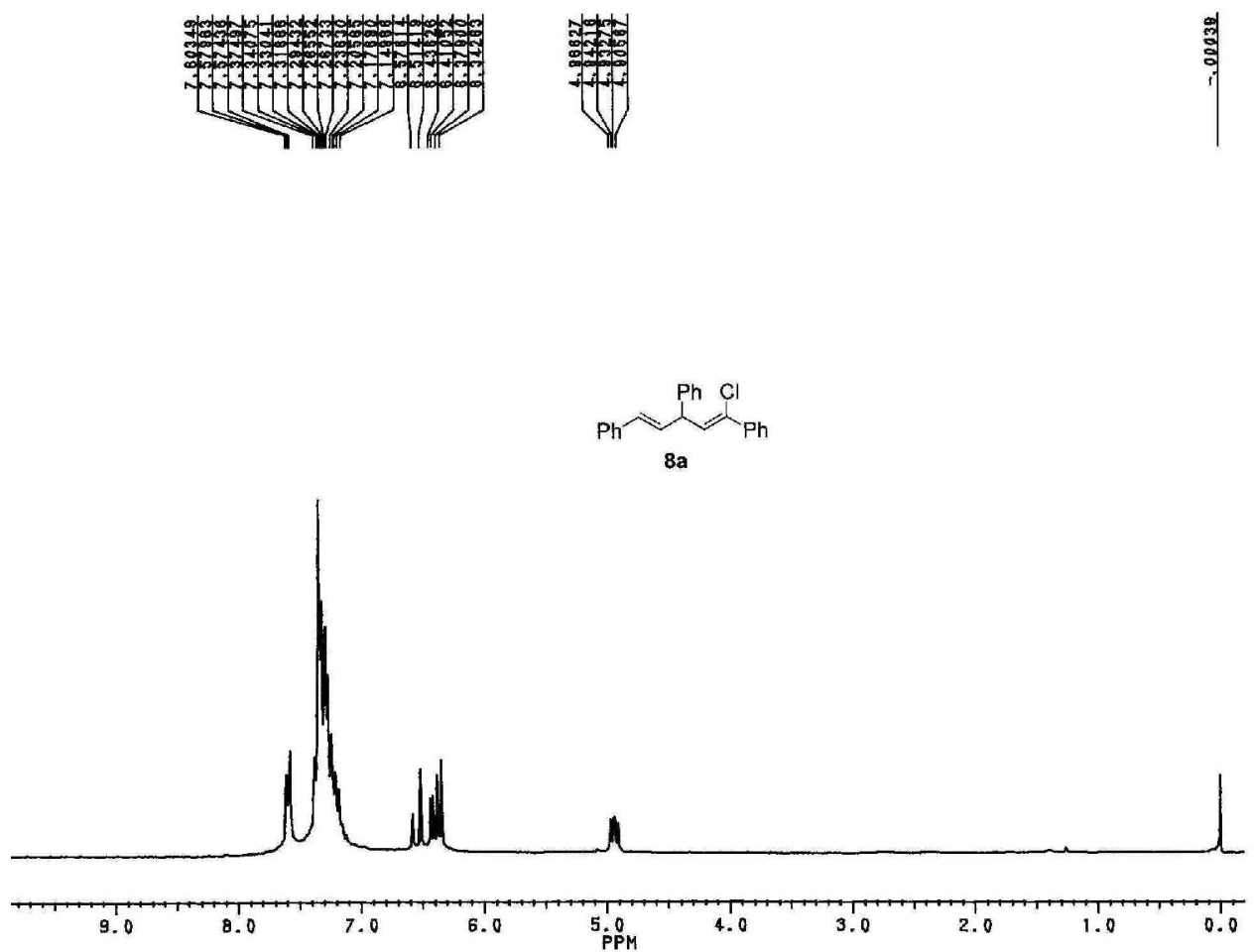
${ }^{13}$ C-NMR spectrum of $8 a$
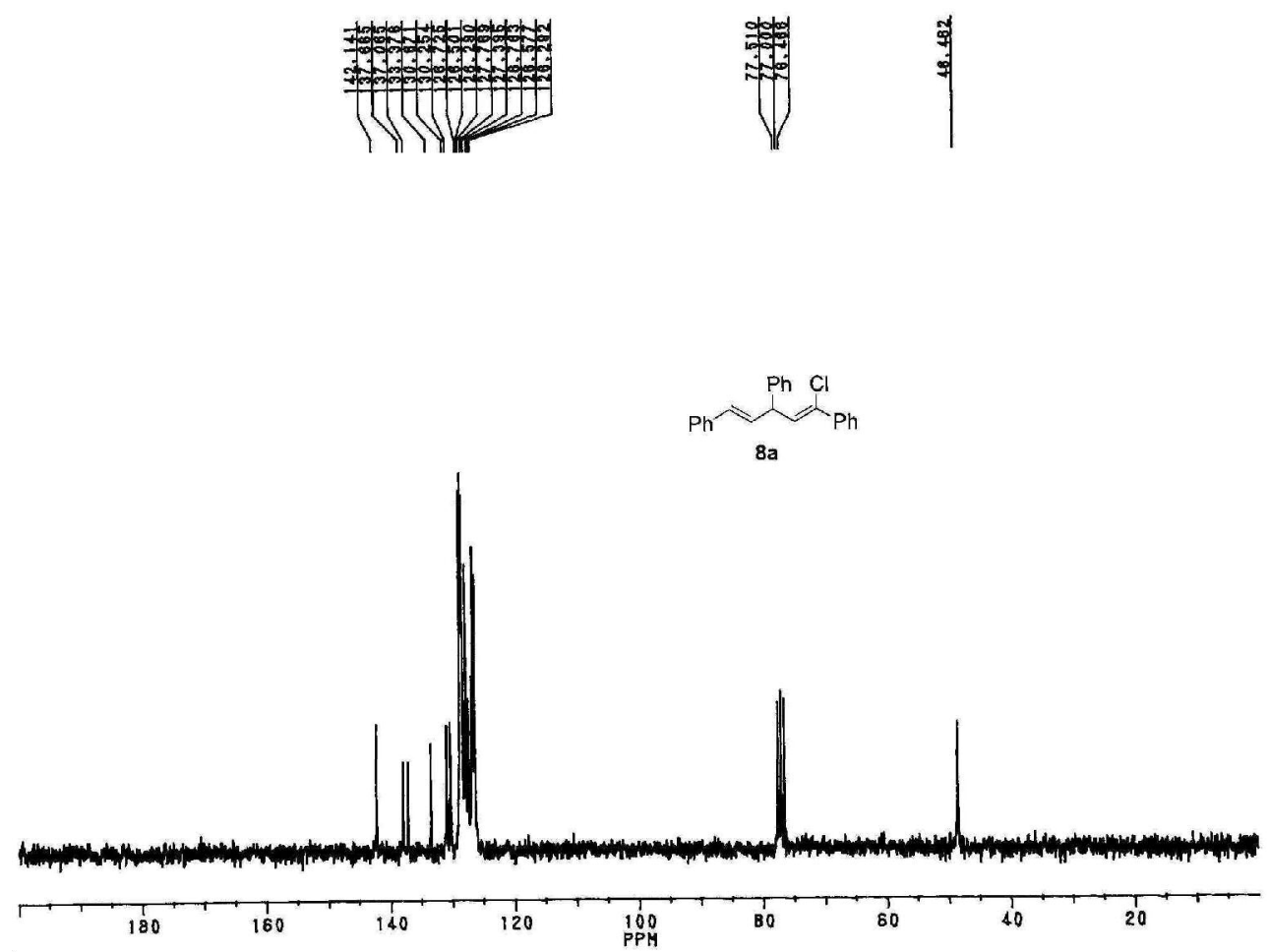


\section{${ }^{1}$ H- NMR spectrum of $8 b$}
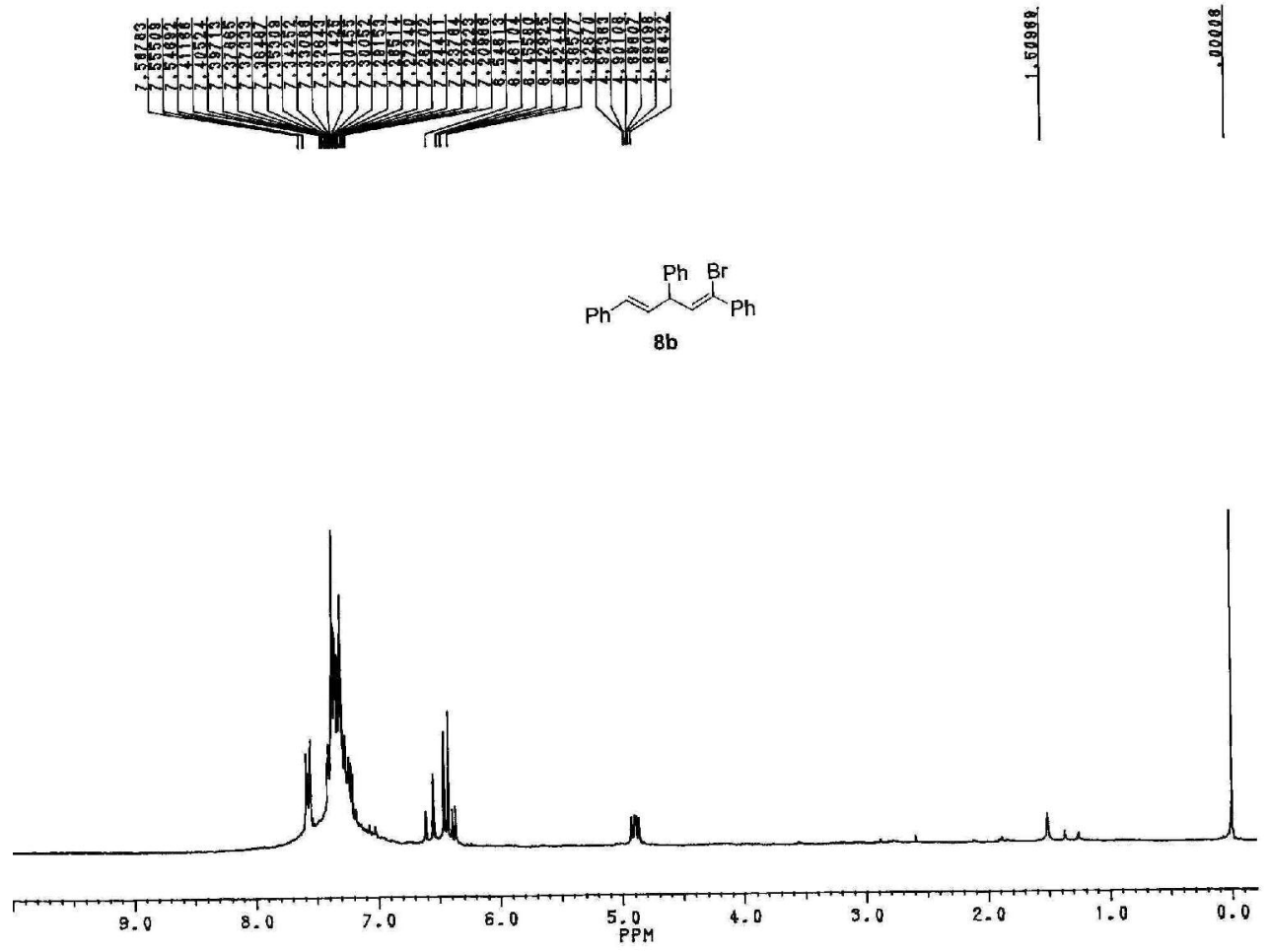
${ }^{13} \mathrm{C}$-NMR spectrum of $8 \mathrm{~b}$
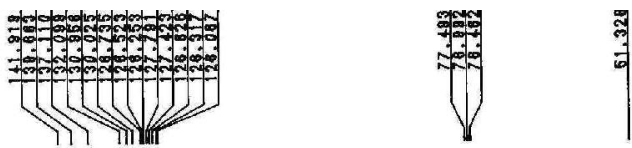

$\underbrace{\mathrm{Ph}}_{8 b} \overbrace{\mathrm{Ph}}^{\mathrm{Br}}$
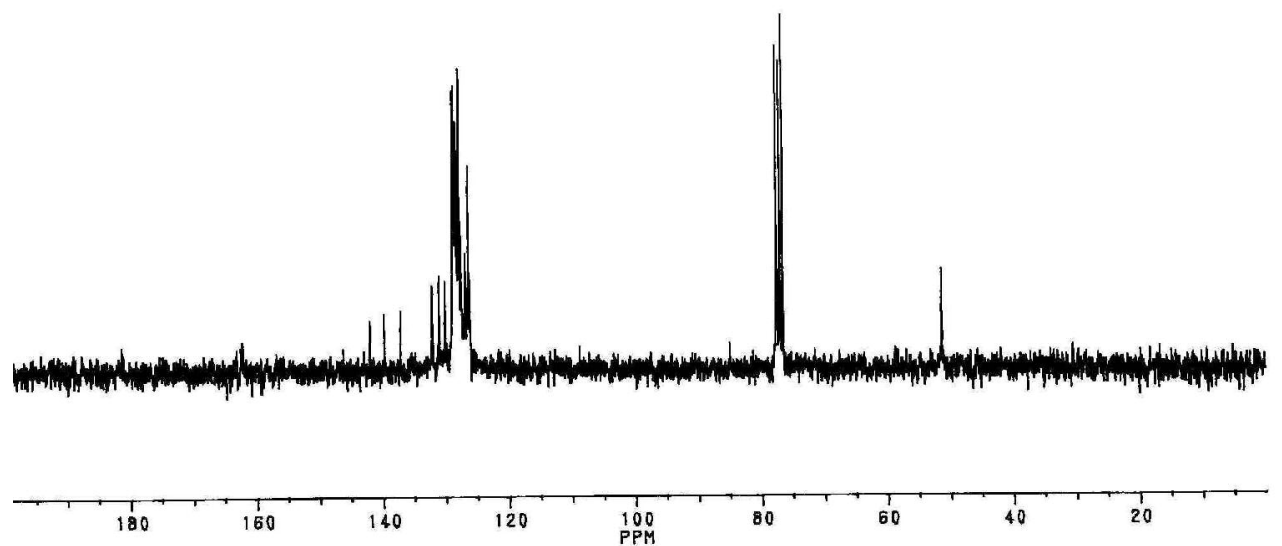


\section{${ }^{1}$ H- NMR spectrum of 9}
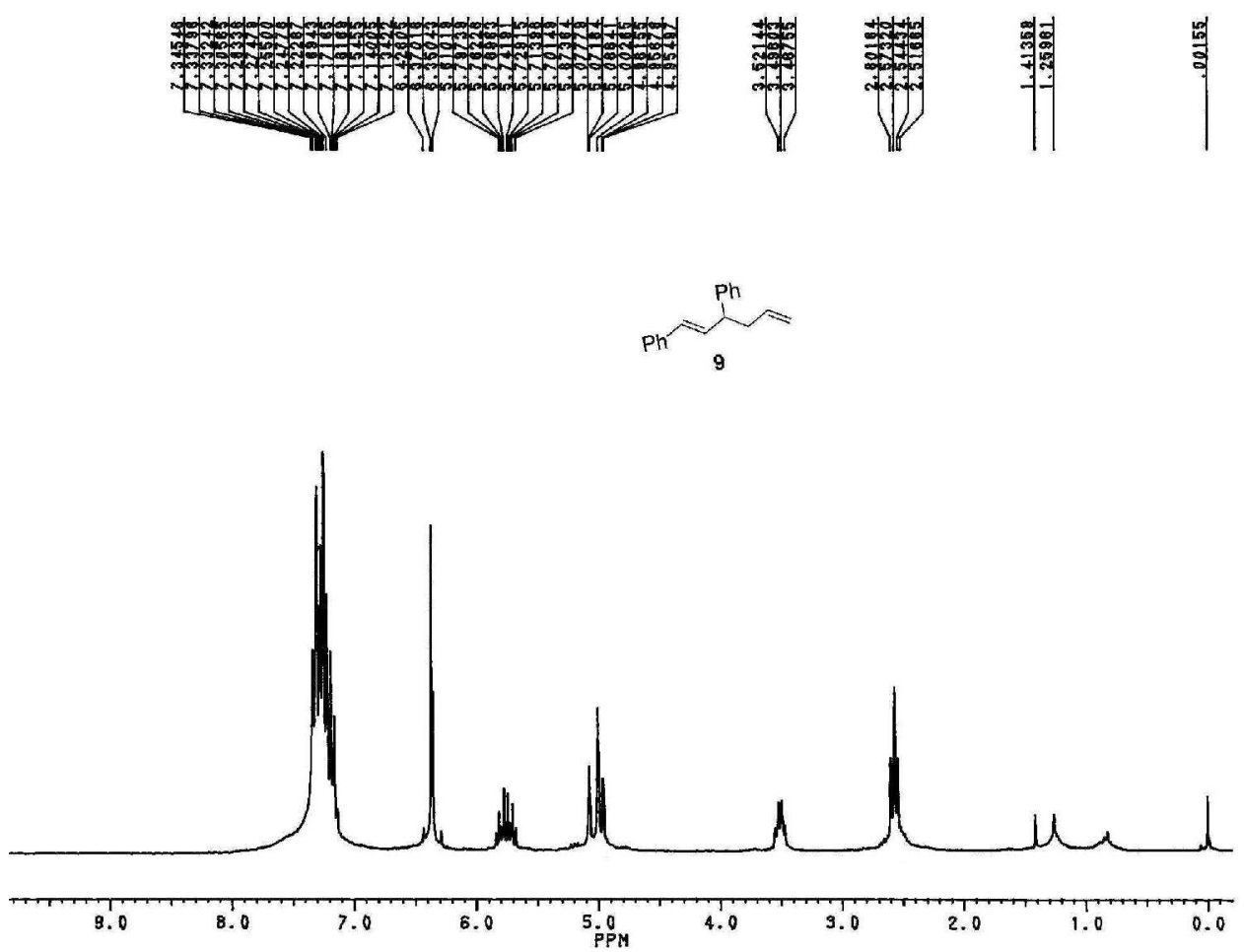


\section{${ }^{13}$ C-NMR spectrum of 9}
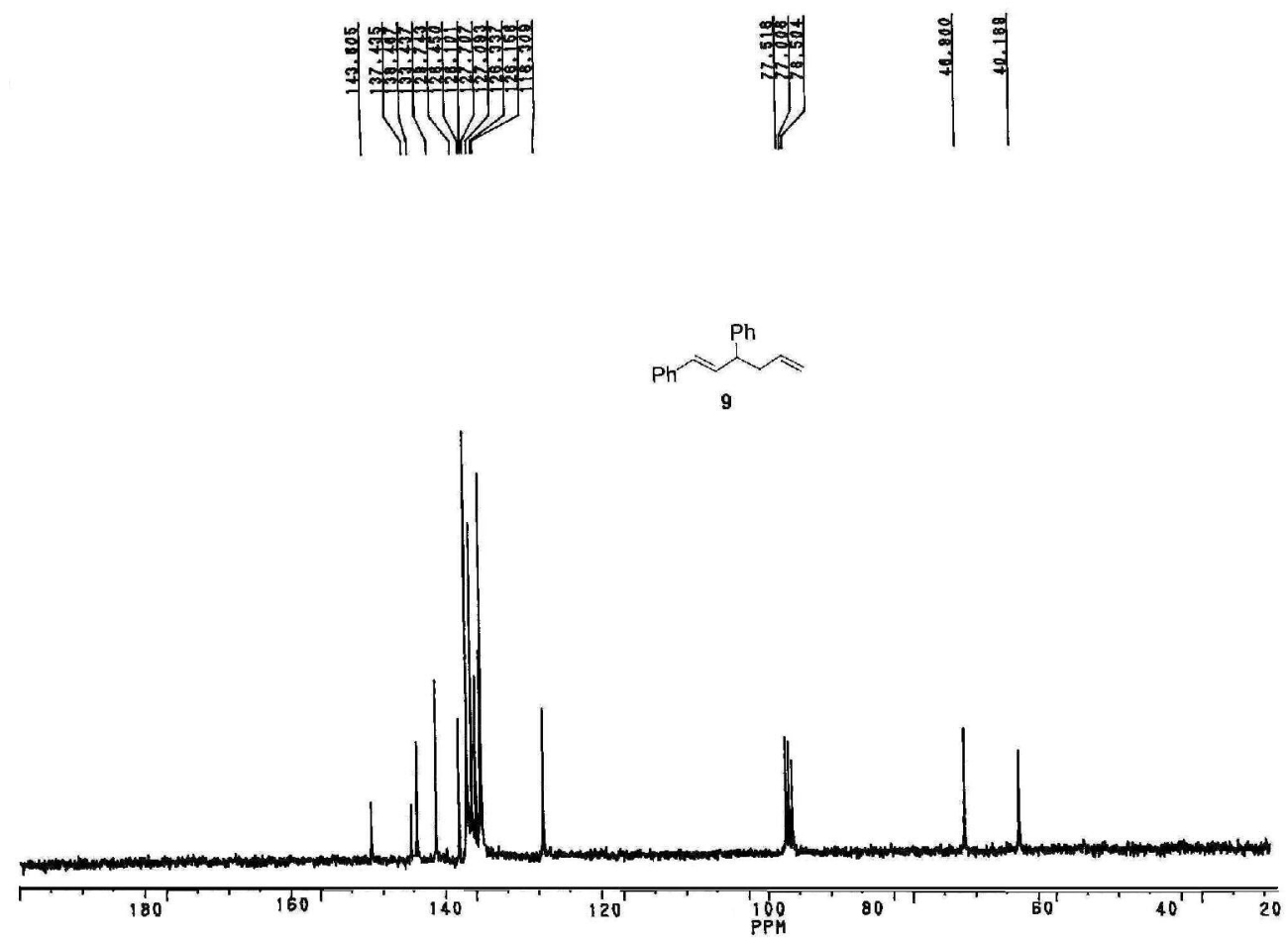


\section{${ }^{1}$ H- NMR spectrum of $10 a$}

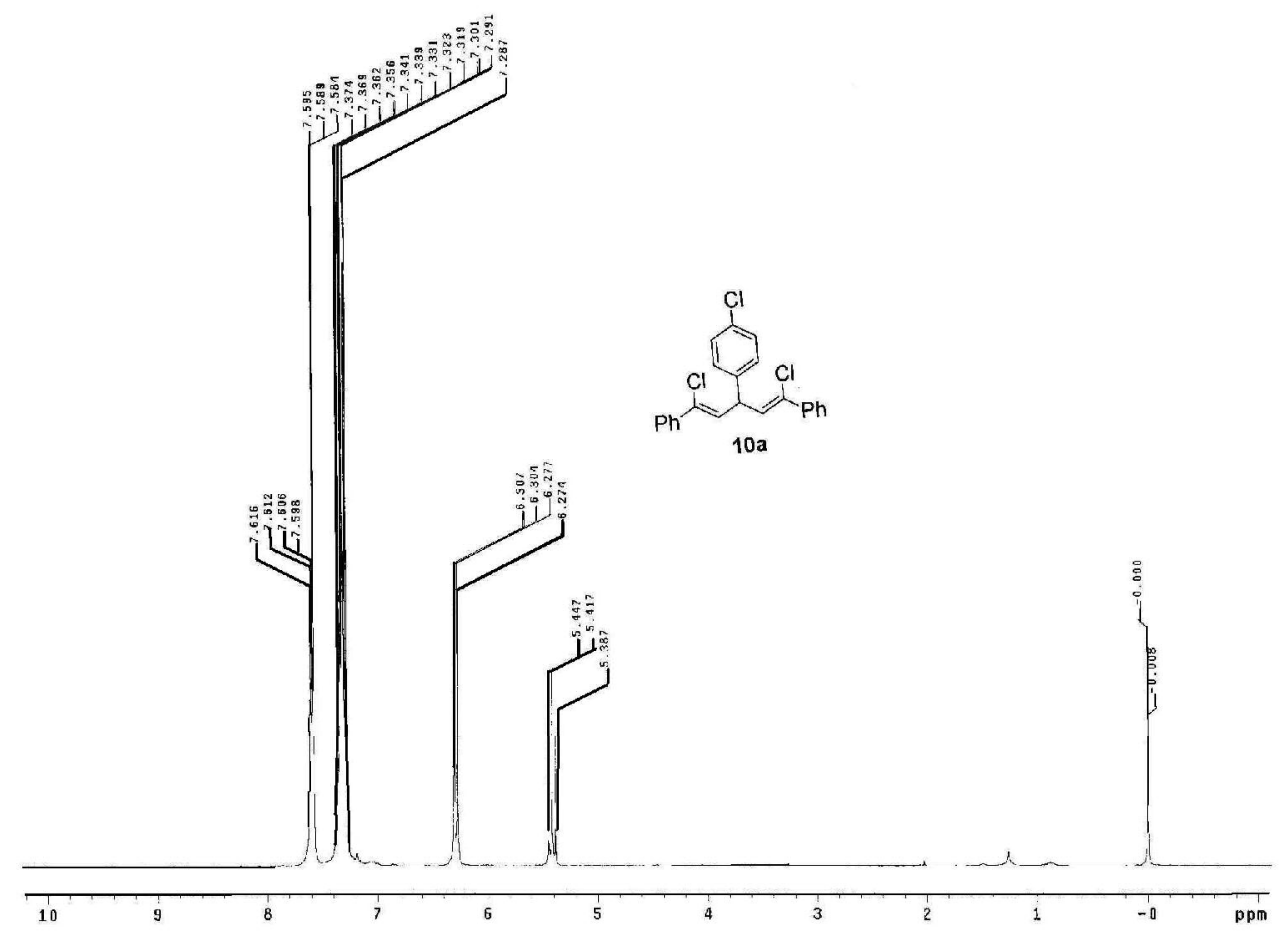




\section{${ }^{13} \mathrm{C}-\mathrm{NMR}$ spectrum of $10 \mathrm{a}$}

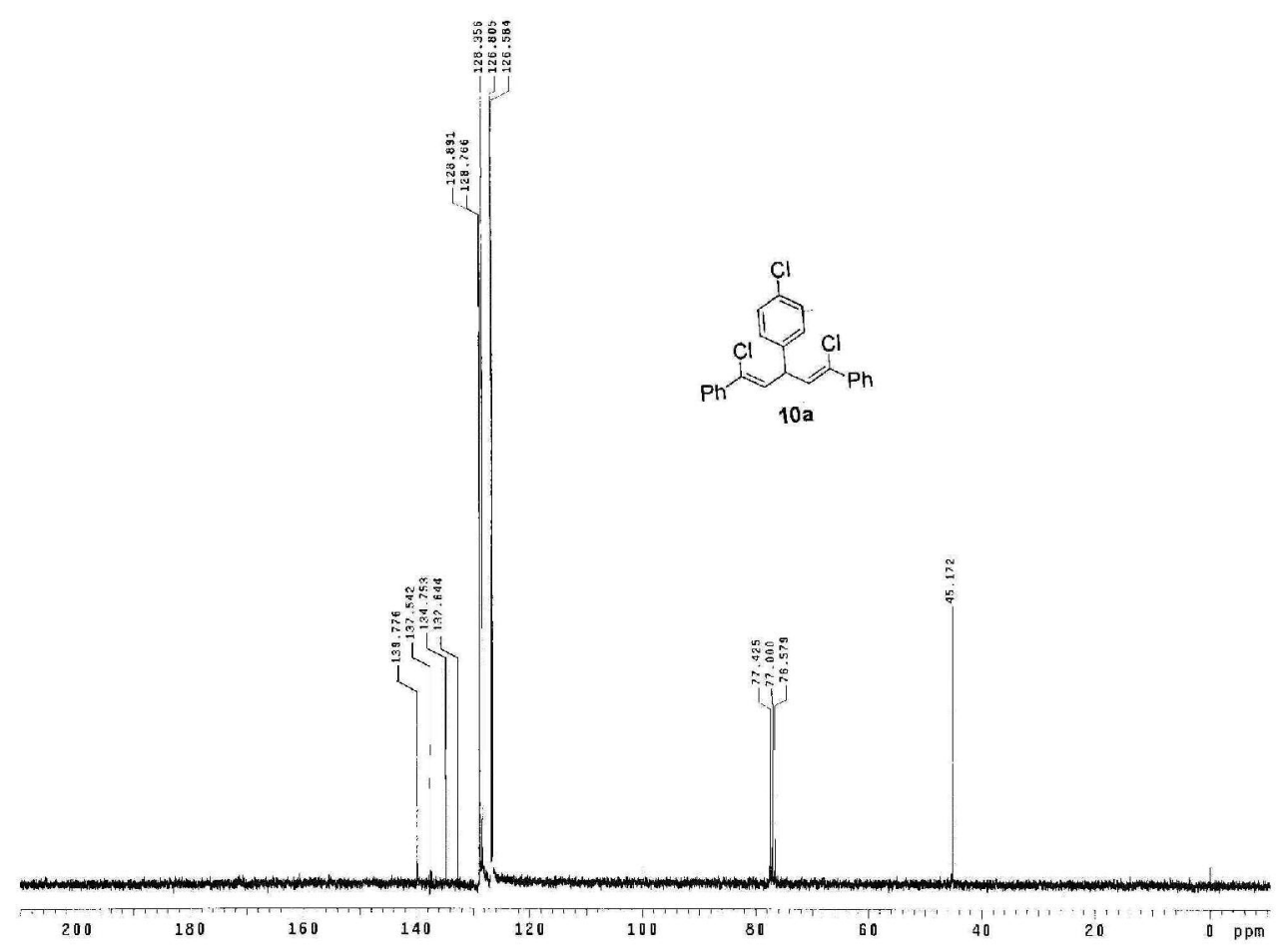




\section{${ }^{1} \mathrm{H}$ - NMR spectrum of $10 \mathrm{~b}$}
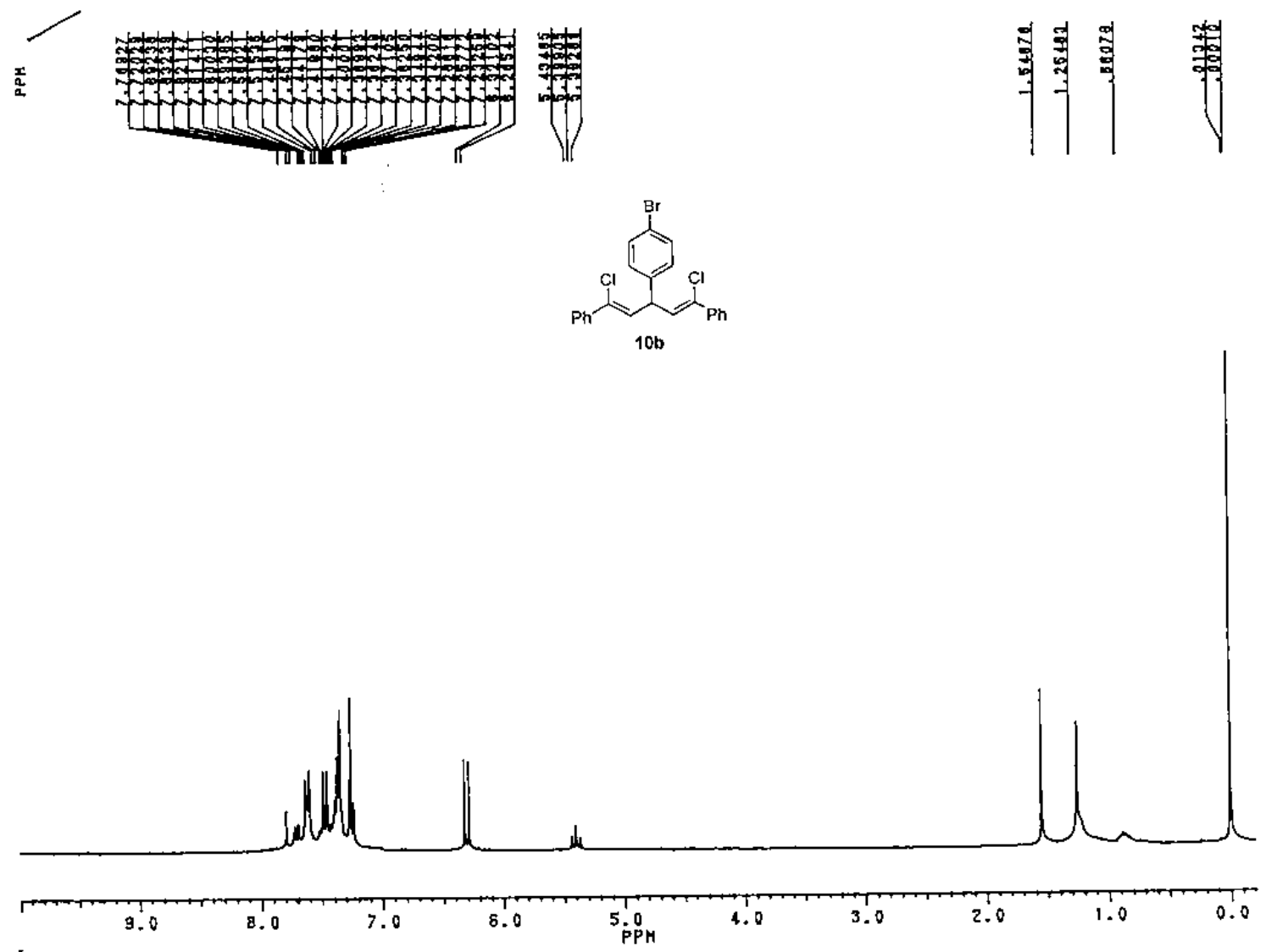
${ }^{13} \mathrm{C}$ - NMR spectrum of $10 \mathrm{~b}$

돌
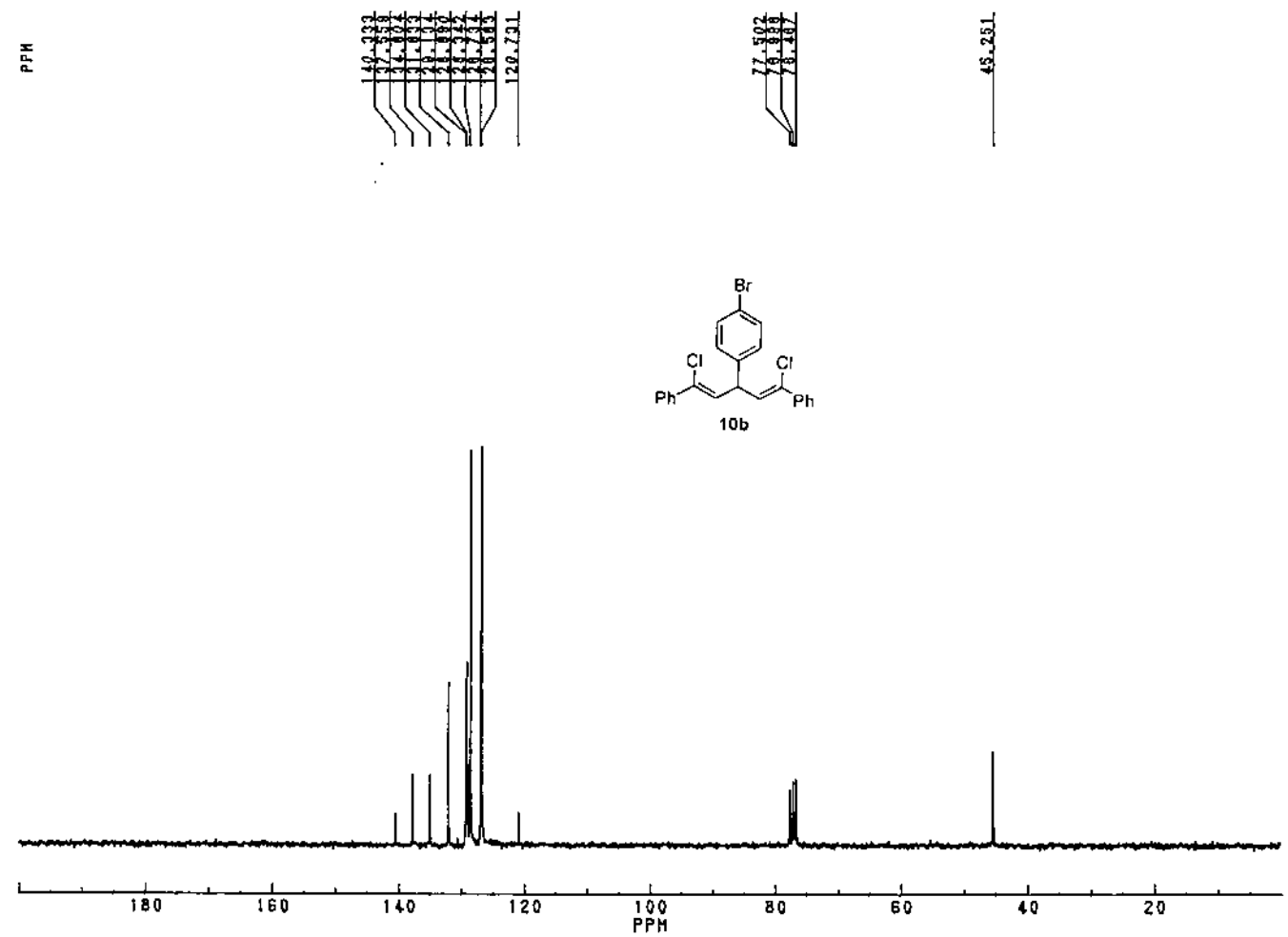


\section{${ }^{1} \mathrm{H}$ - NMR spectrum of $10 \mathrm{c}$}

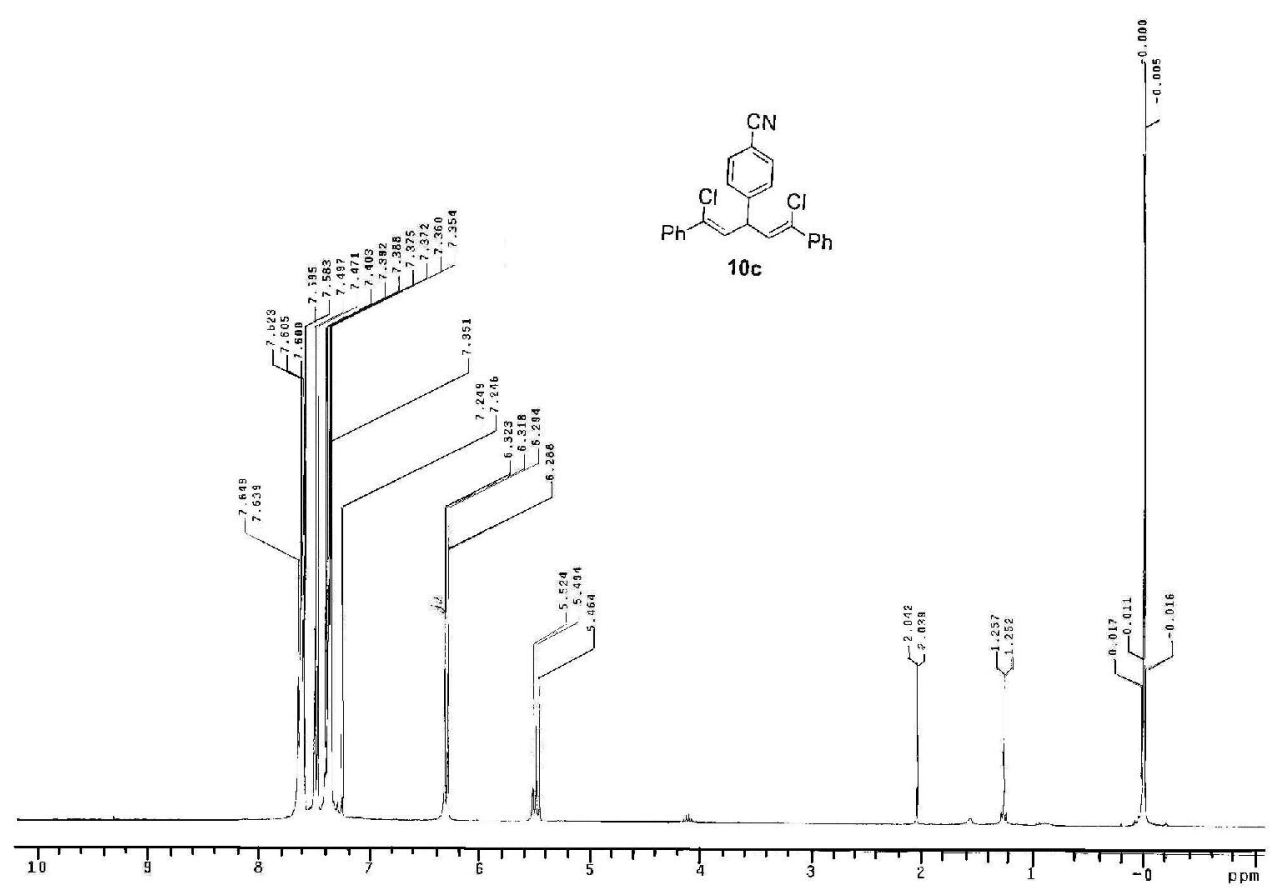




\section{${ }^{13} \mathrm{C}-\mathrm{NMR}$ spectrum of $10 \mathrm{c}$}

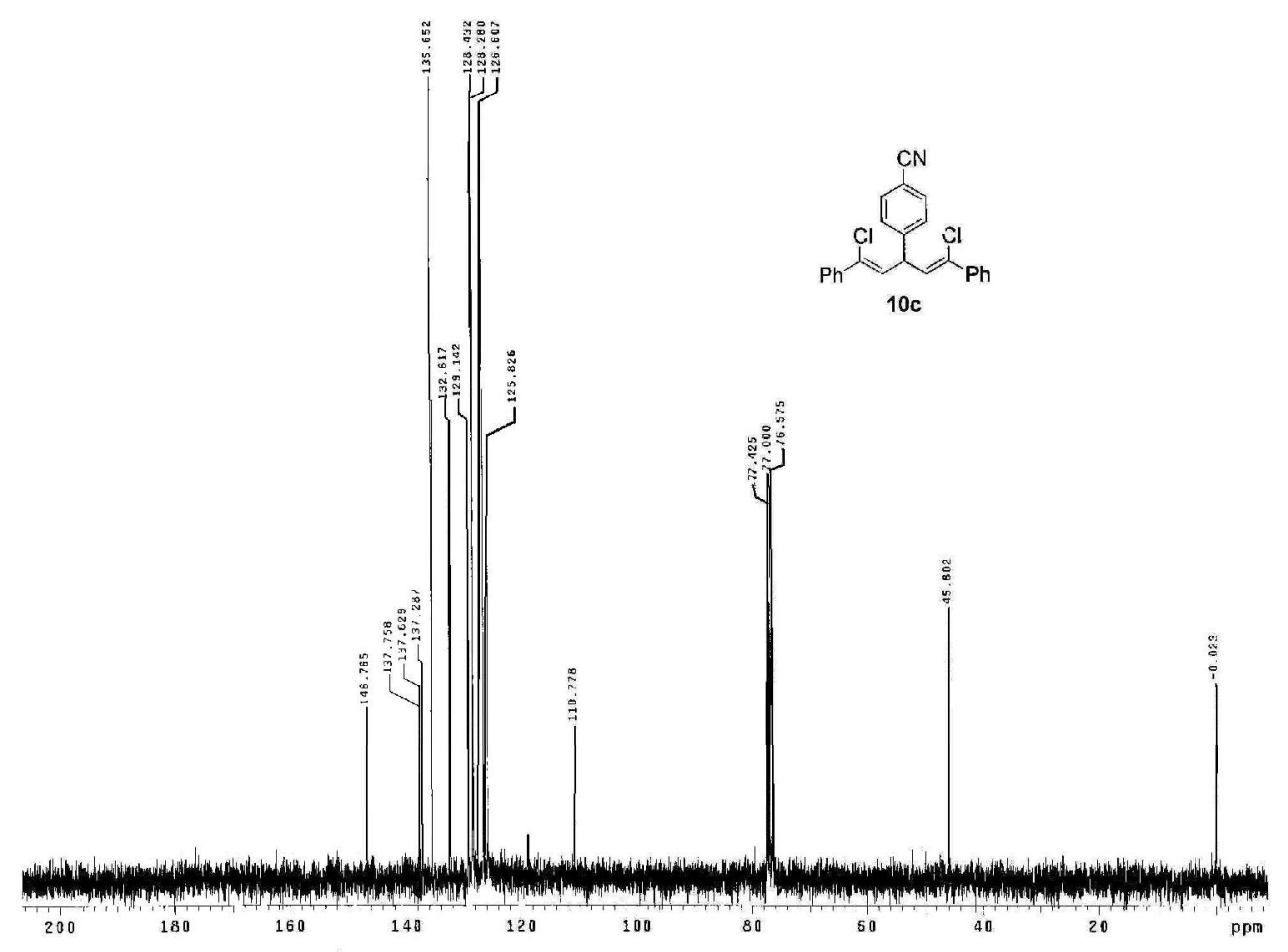




\section{${ }^{1} \mathrm{H}$ - NMR spectrum of $10 \mathrm{~d}$}

/)

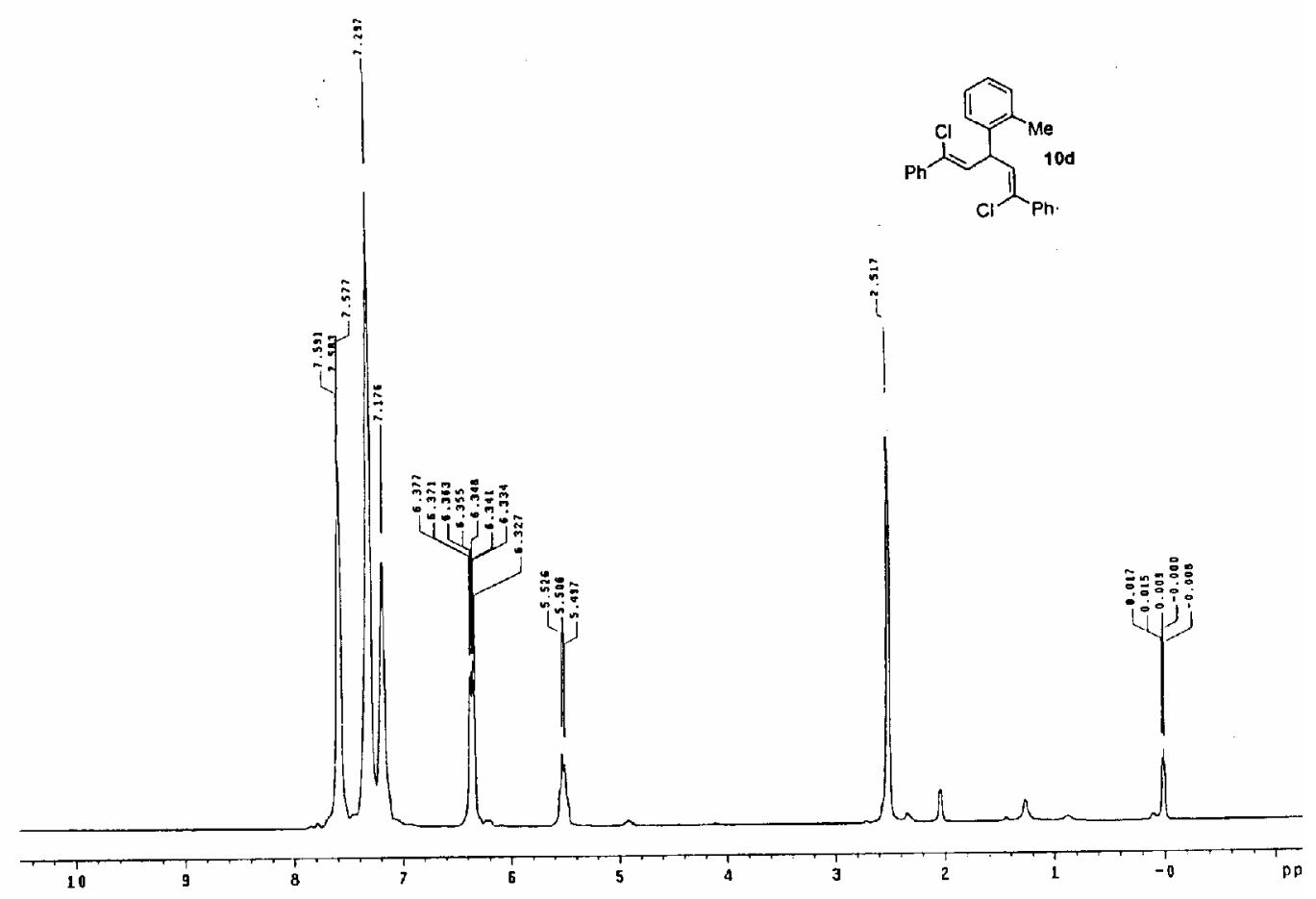


${ }^{13}$ C- NMR spectrum of $10 d$

$r$

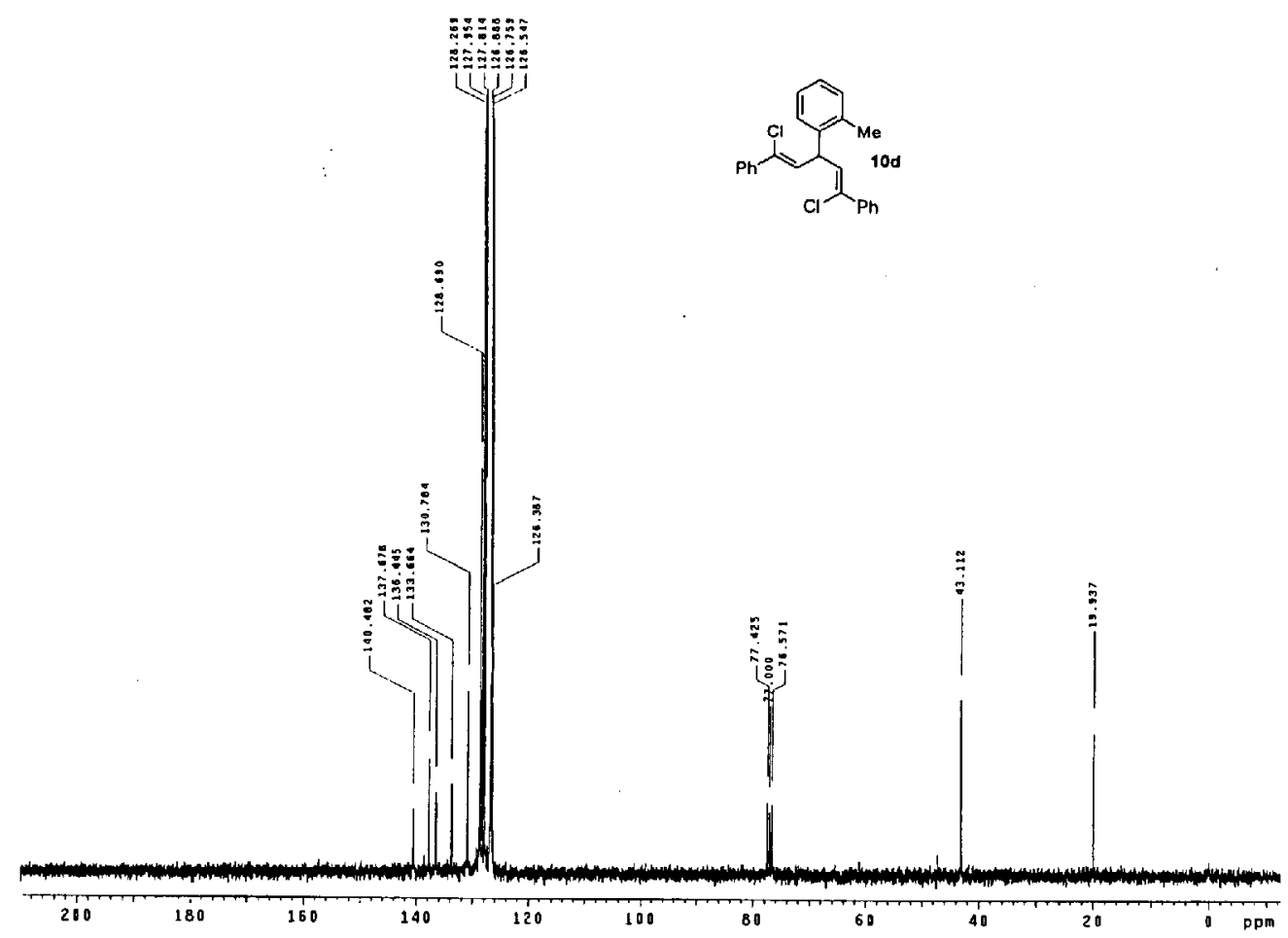




\section{${ }^{1} \mathrm{H}$ - NMR spectrum of $10 \mathrm{e}$}
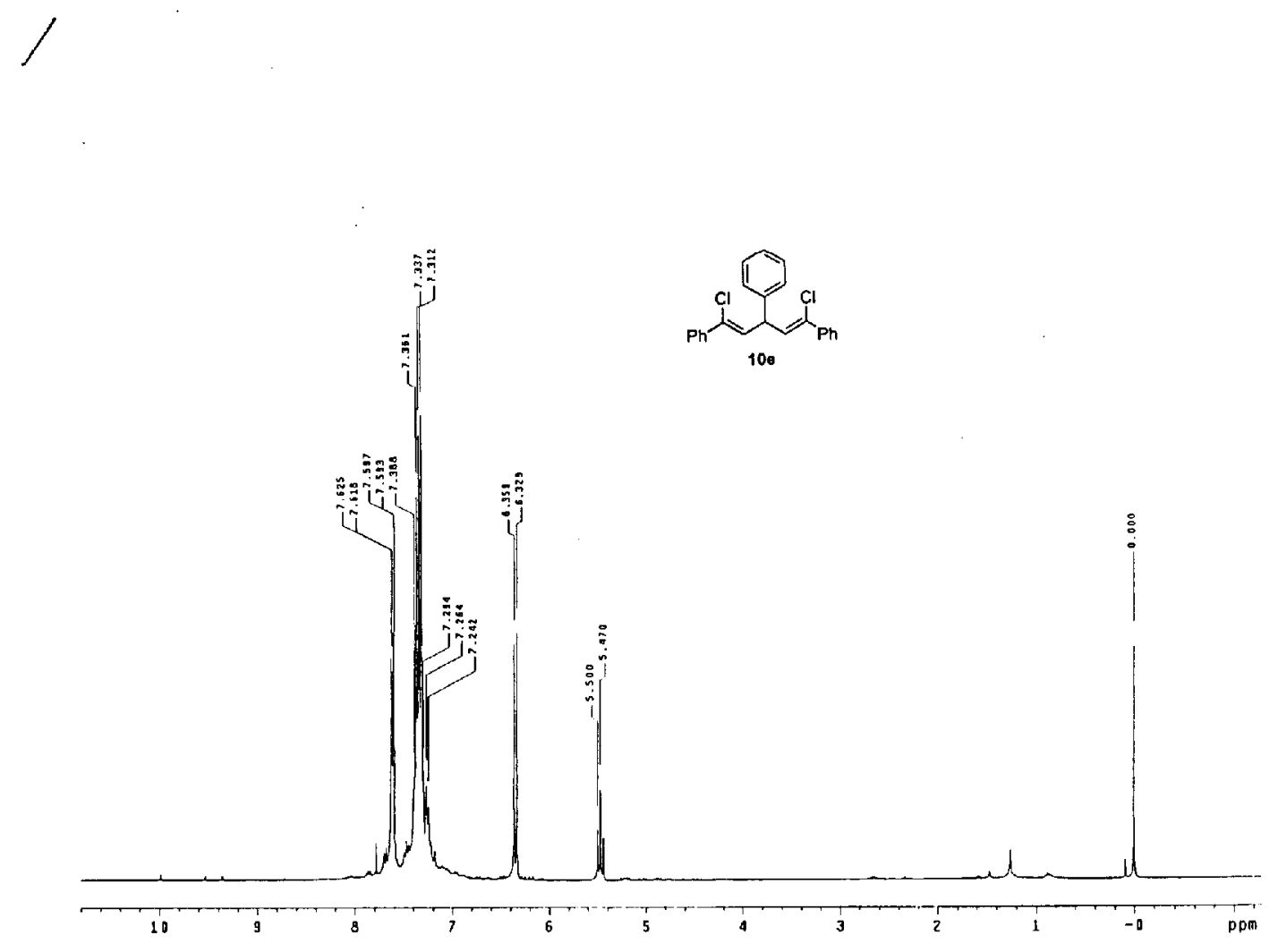
${ }^{13} \mathrm{C}$ - NMR spectrum of $10 \mathrm{e}$

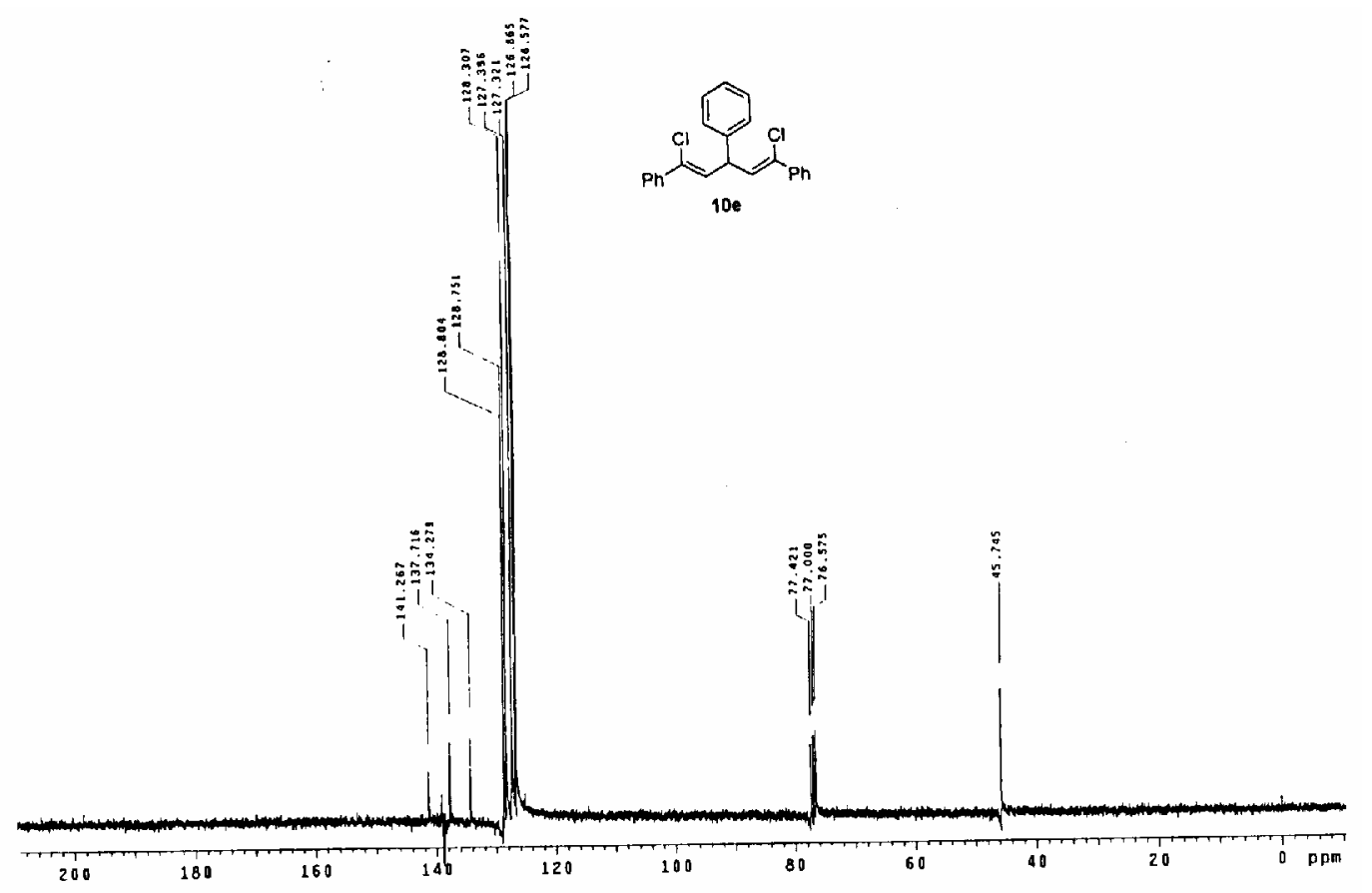




\section{${ }^{1} \mathrm{H}$ - NMR spectrum of $10 \mathrm{f}$}
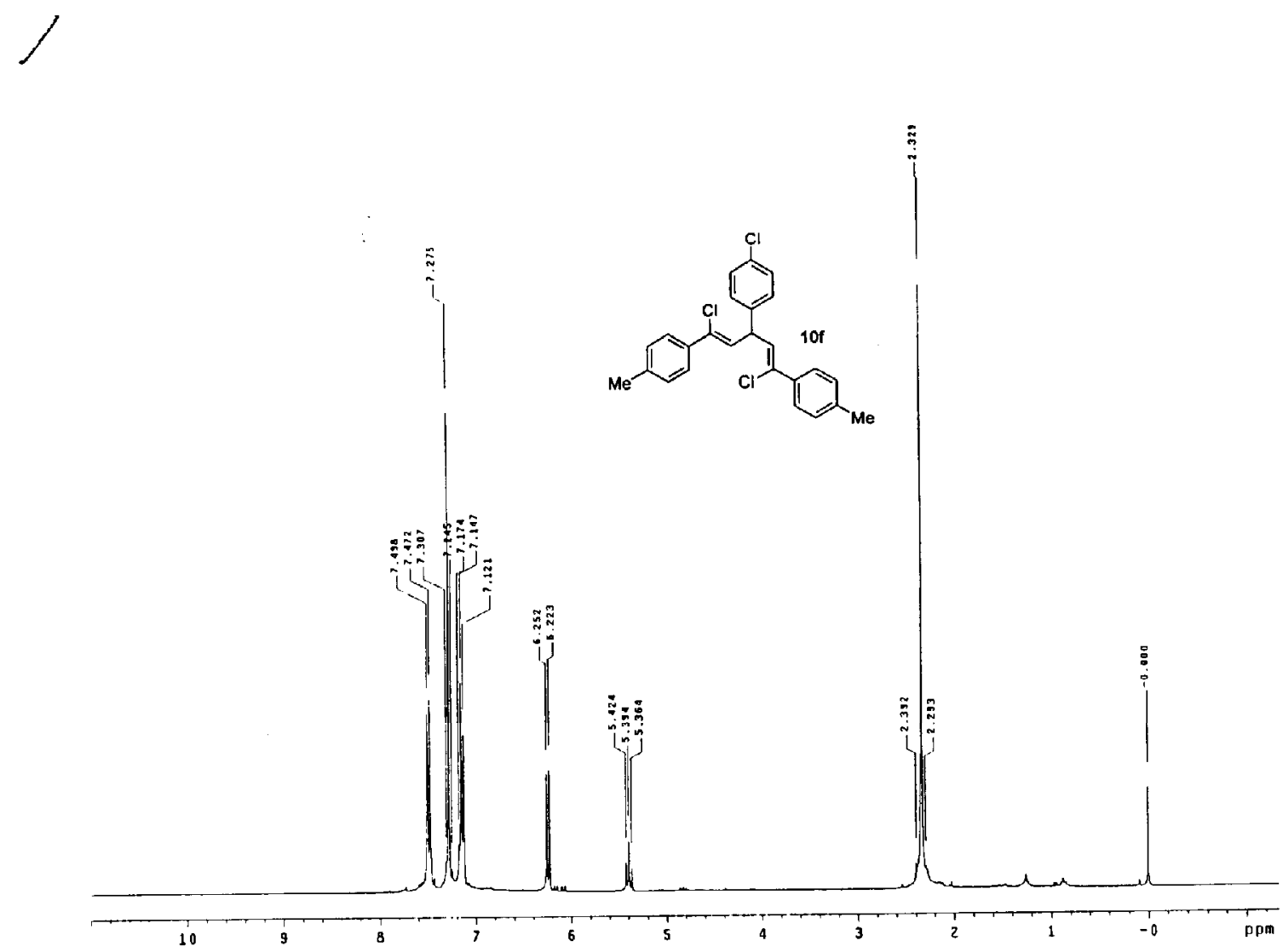


\section{${ }^{13} \mathrm{C}$ - NMR spectrum of $10 \mathrm{f}$}

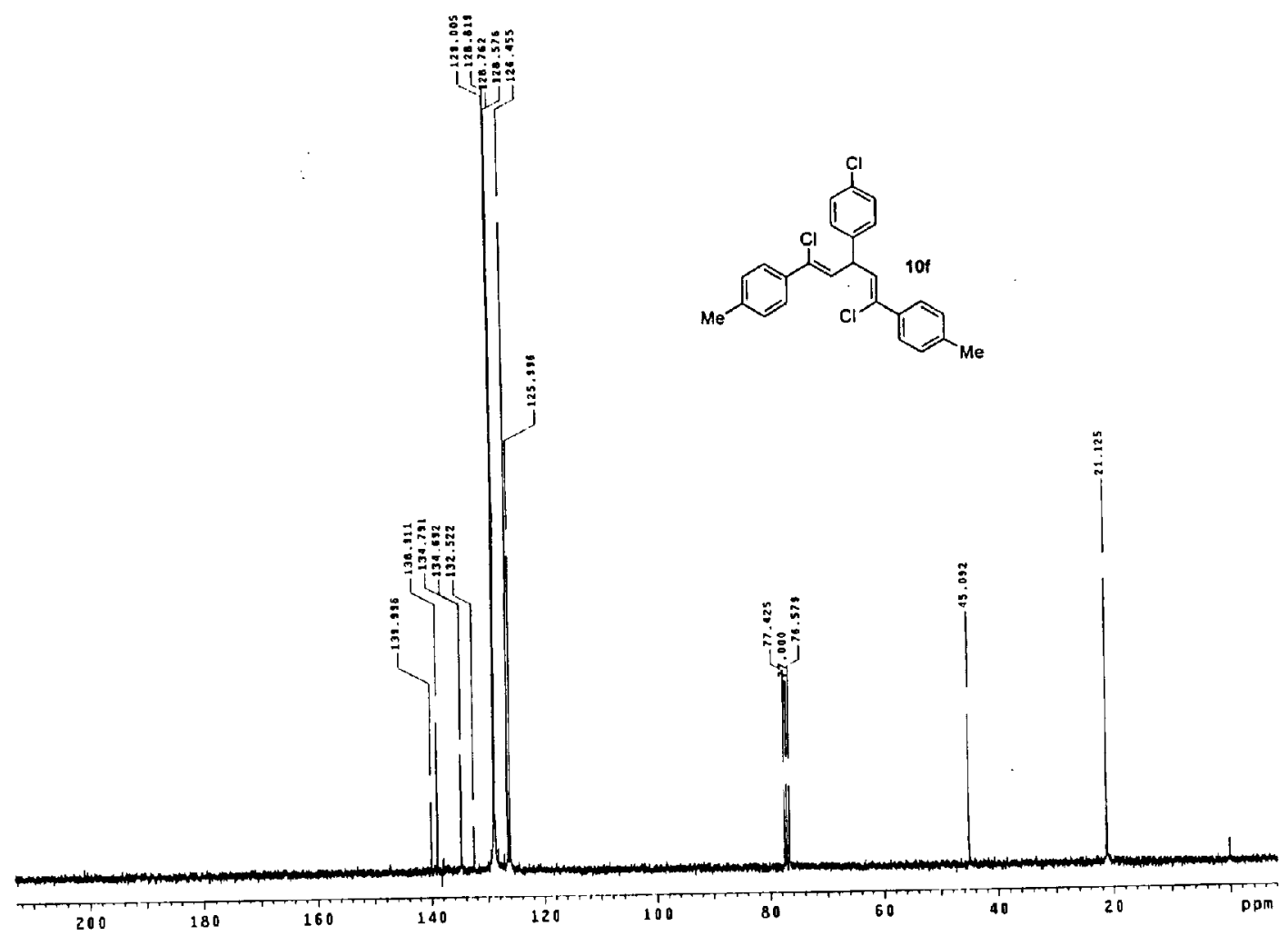




\section{${ }^{1} \mathrm{H}$ - NMR spectrum of $10 \mathrm{~g}$}

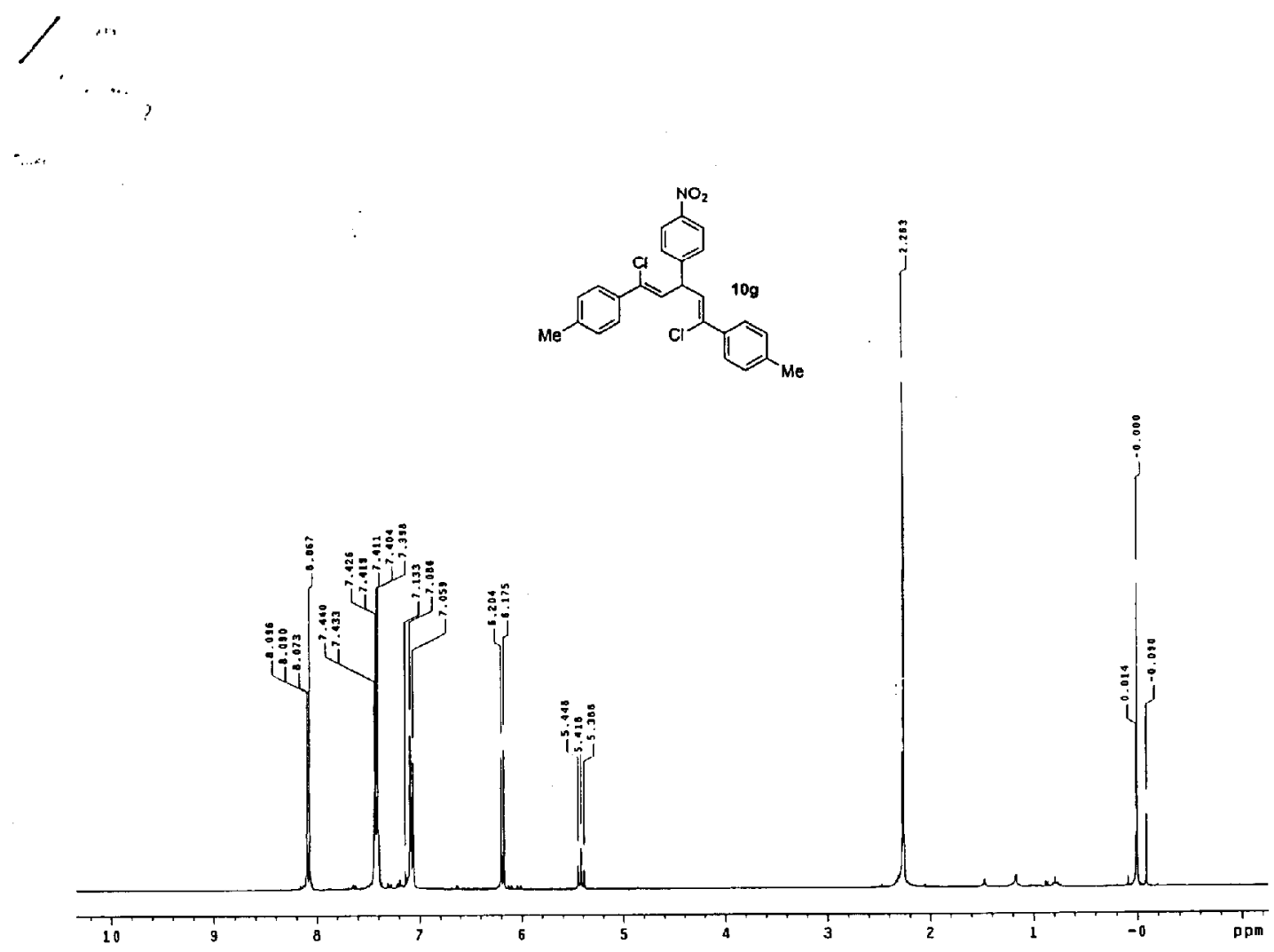


${ }^{13} \mathrm{C}$ - NMR spectrum of $10 \mathrm{~g}$

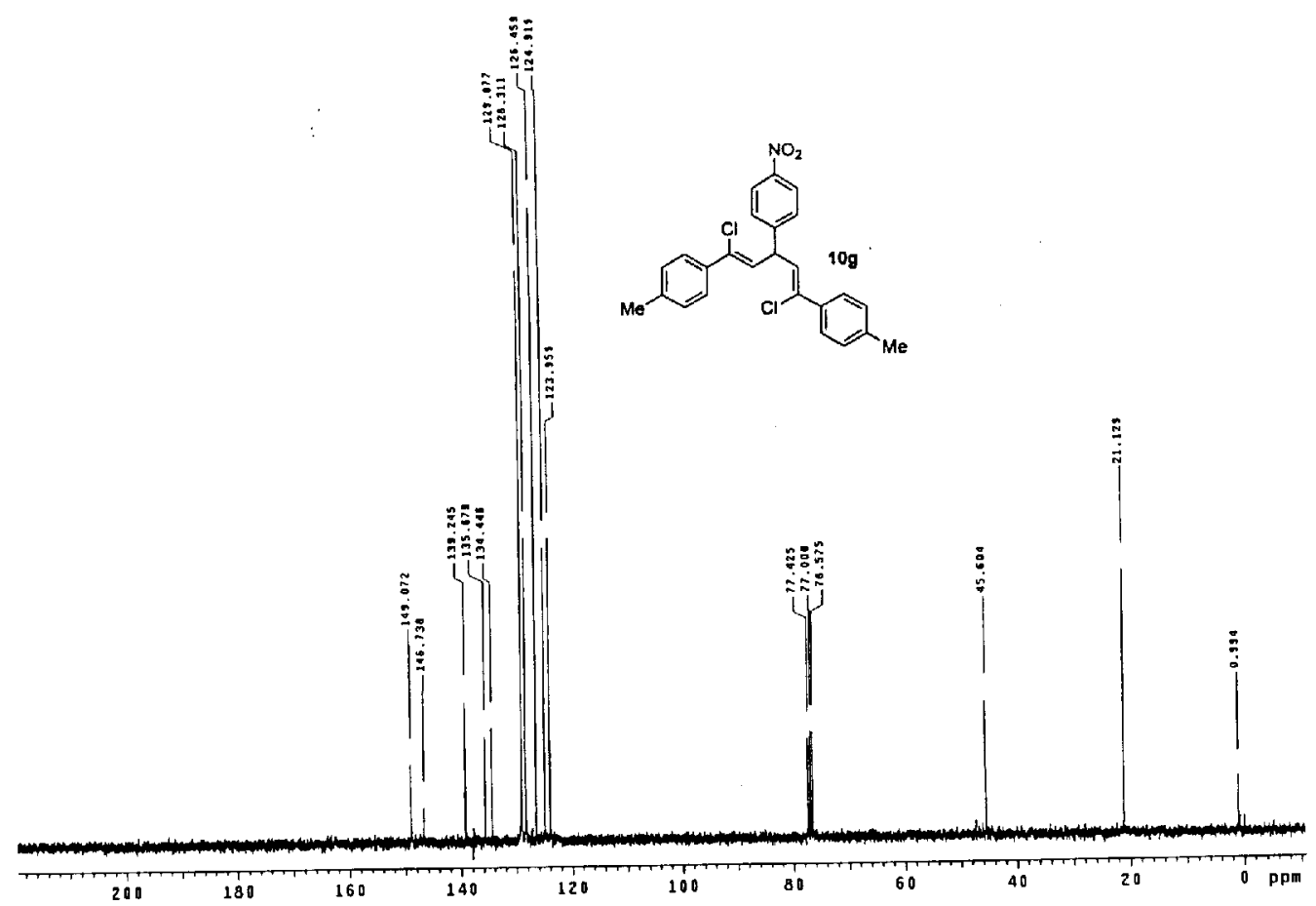




\section{${ }^{1} \mathrm{H}$ - NMR spectrum of $10 \mathrm{~h}$}

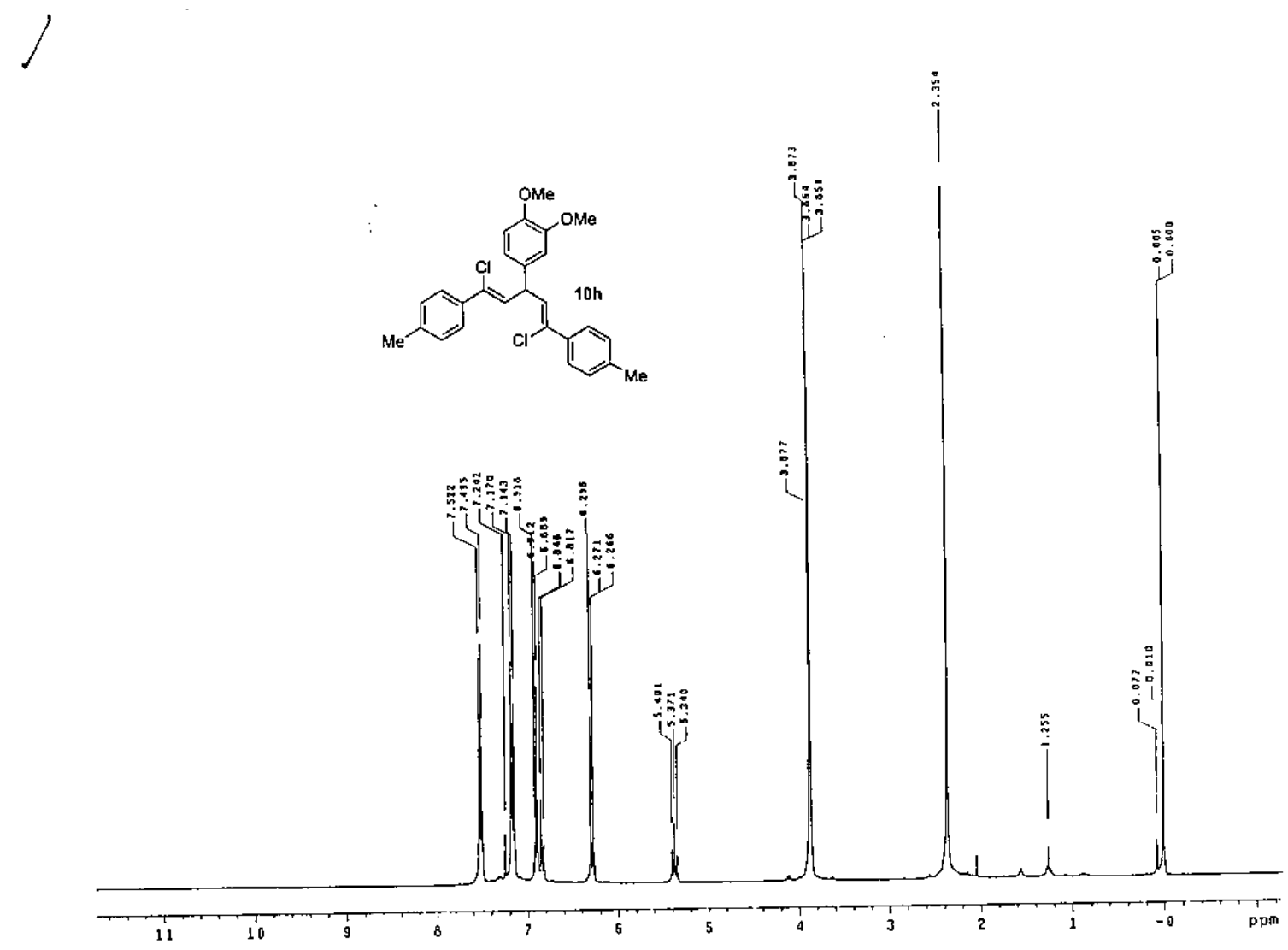


${ }^{13} \mathrm{C}$ - NMR spectrum of $10 \mathrm{~h}$

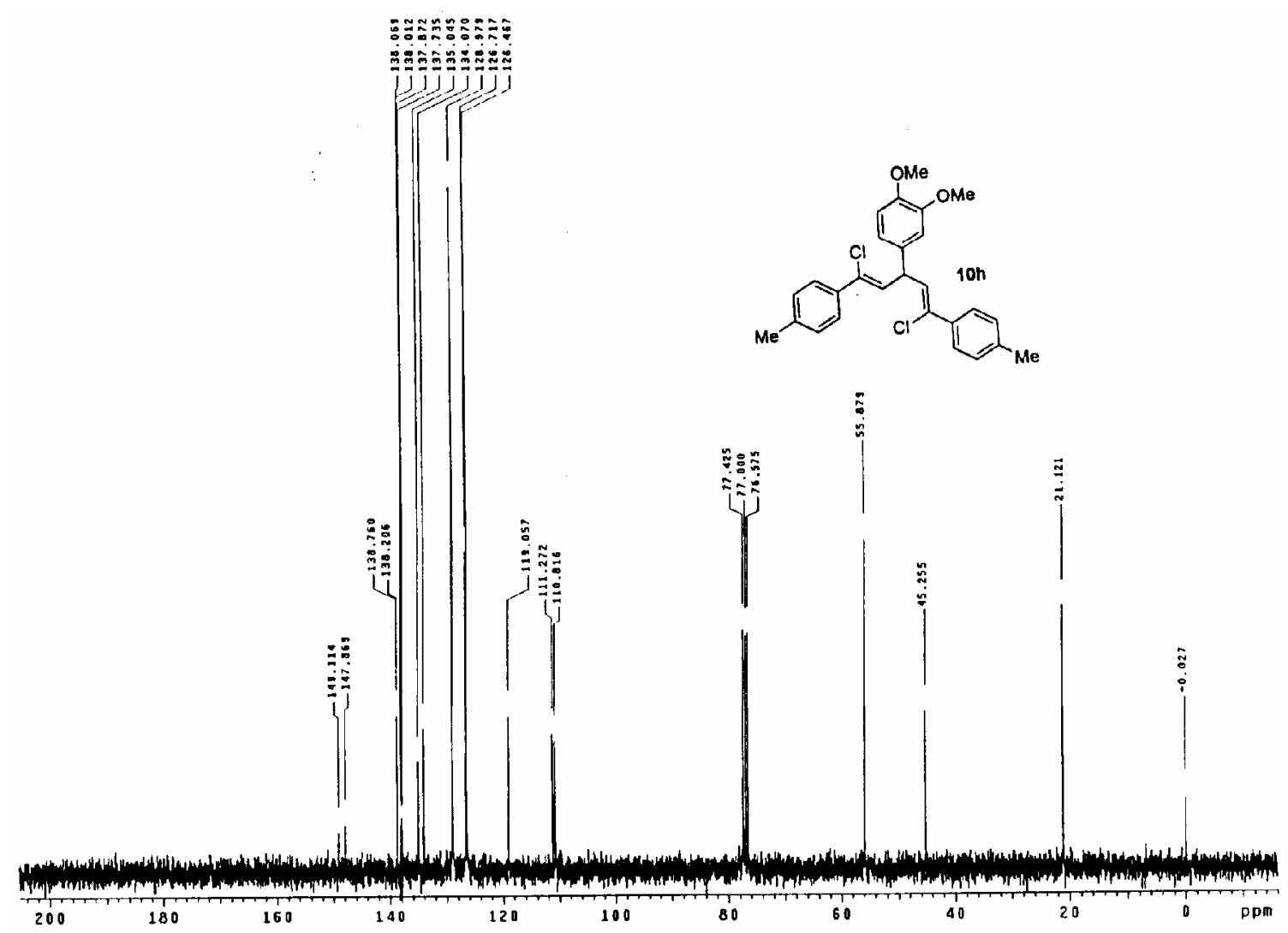




\section{${ }^{1} \mathrm{H}$ - NMR spectrum of $10 \mathrm{i}$}

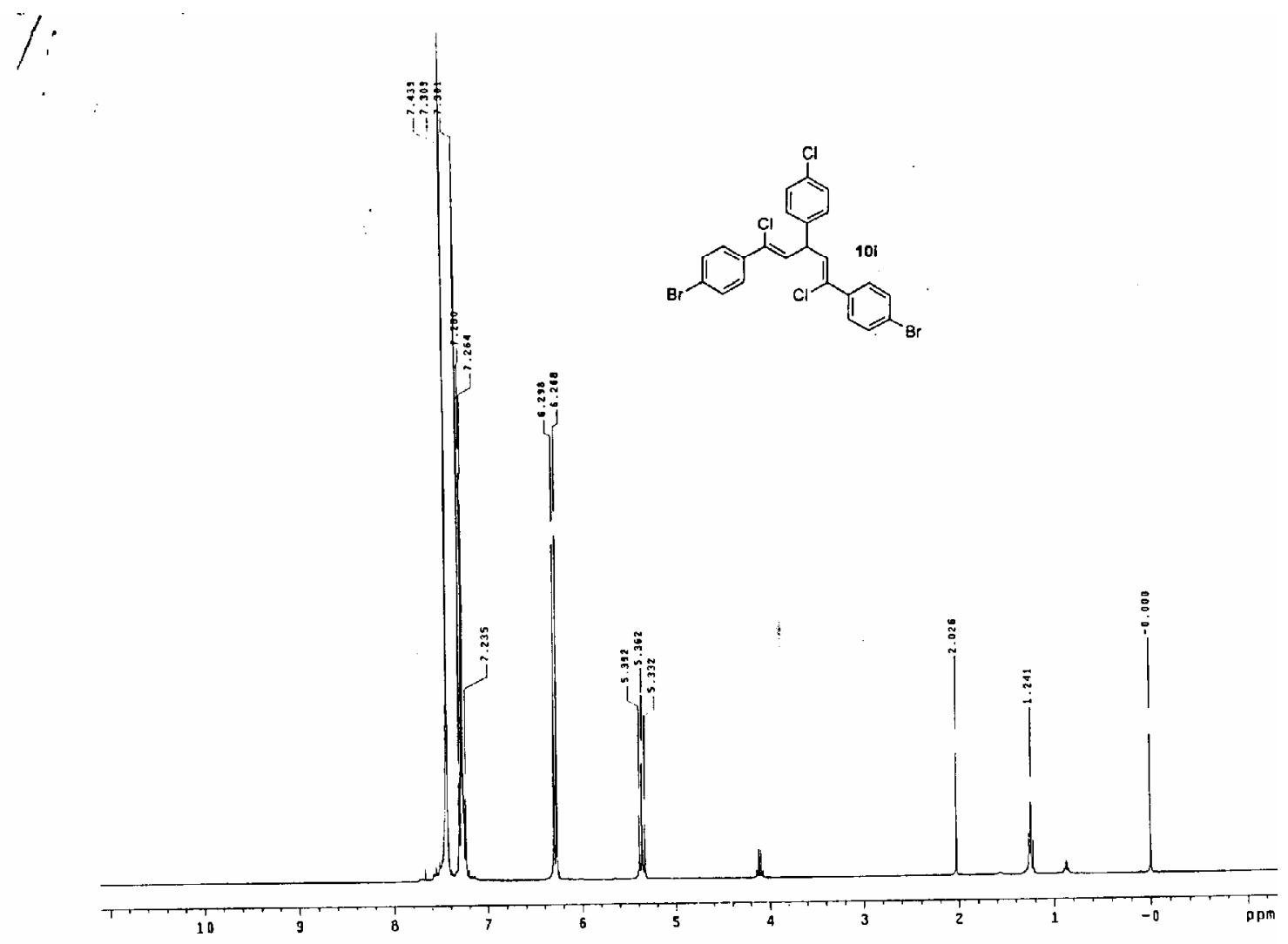




\section{${ }^{13} \mathrm{C}$ - NMR spectrum of $10 \mathrm{i}$}

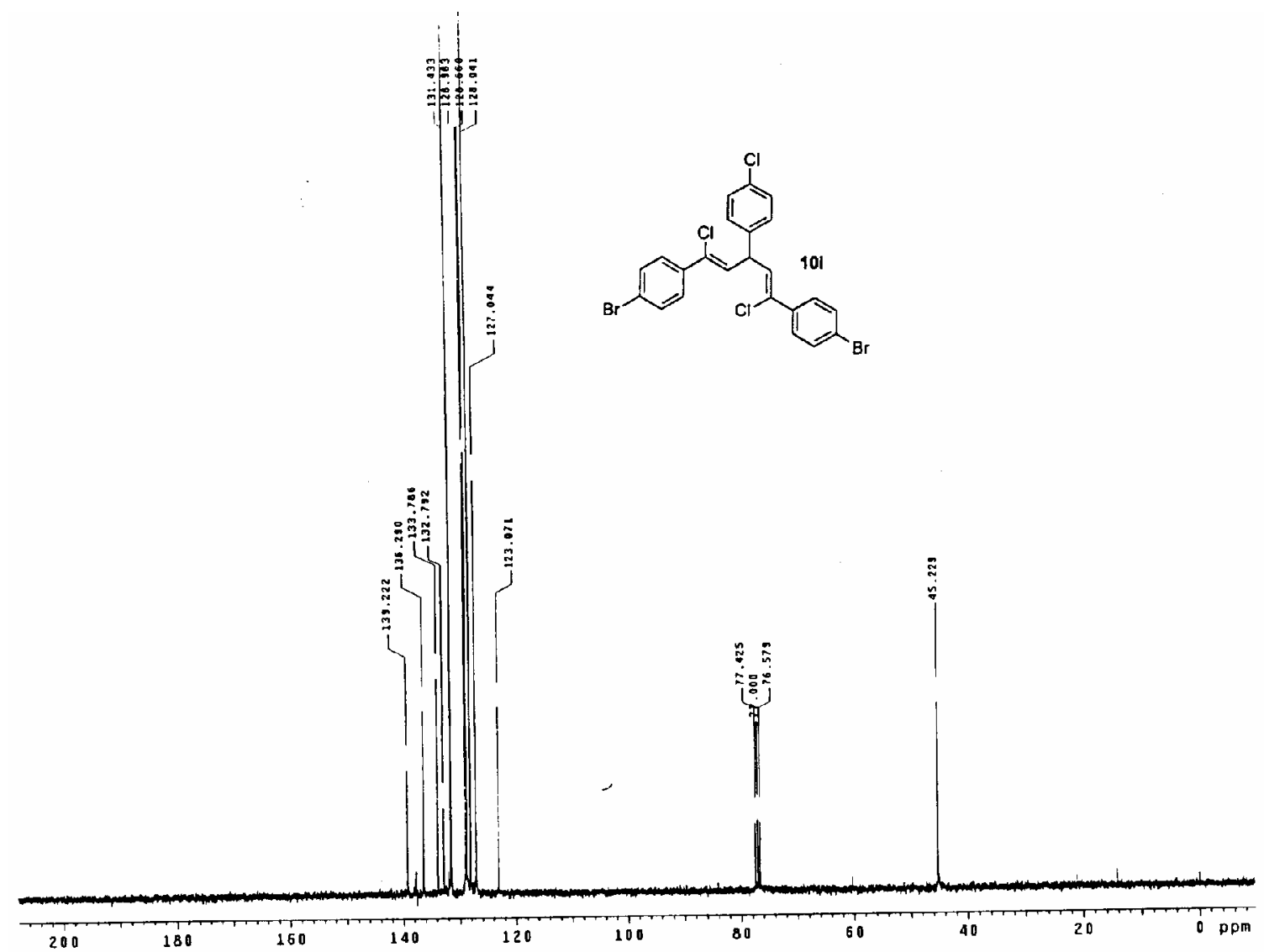




\section{${ }^{1} \mathrm{H}$ - NMR spectrum of $\mathbf{1 0 j}$}

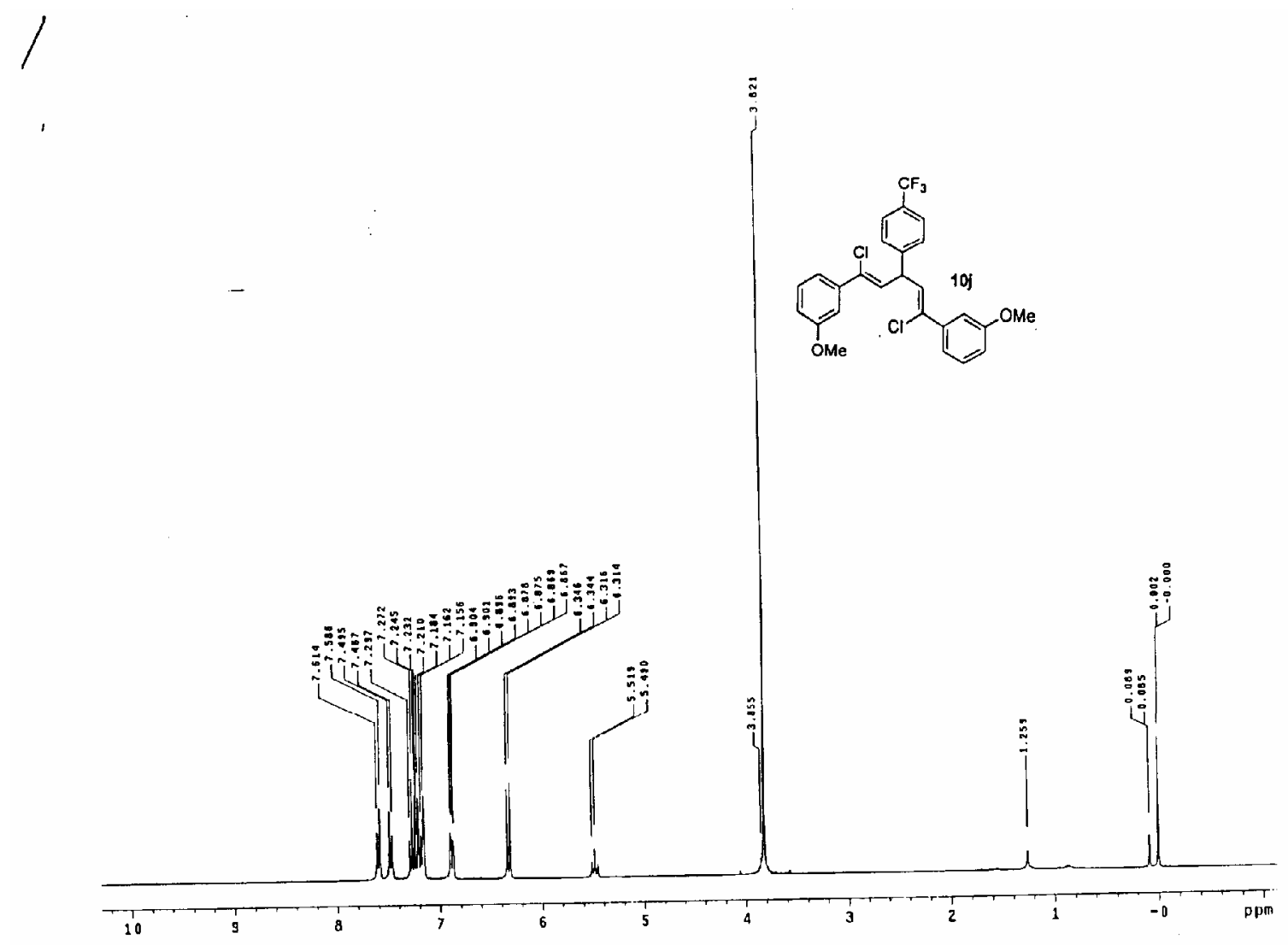


${ }^{13} \mathrm{C}$ - NMR spectrum of $10 \mathrm{j}$

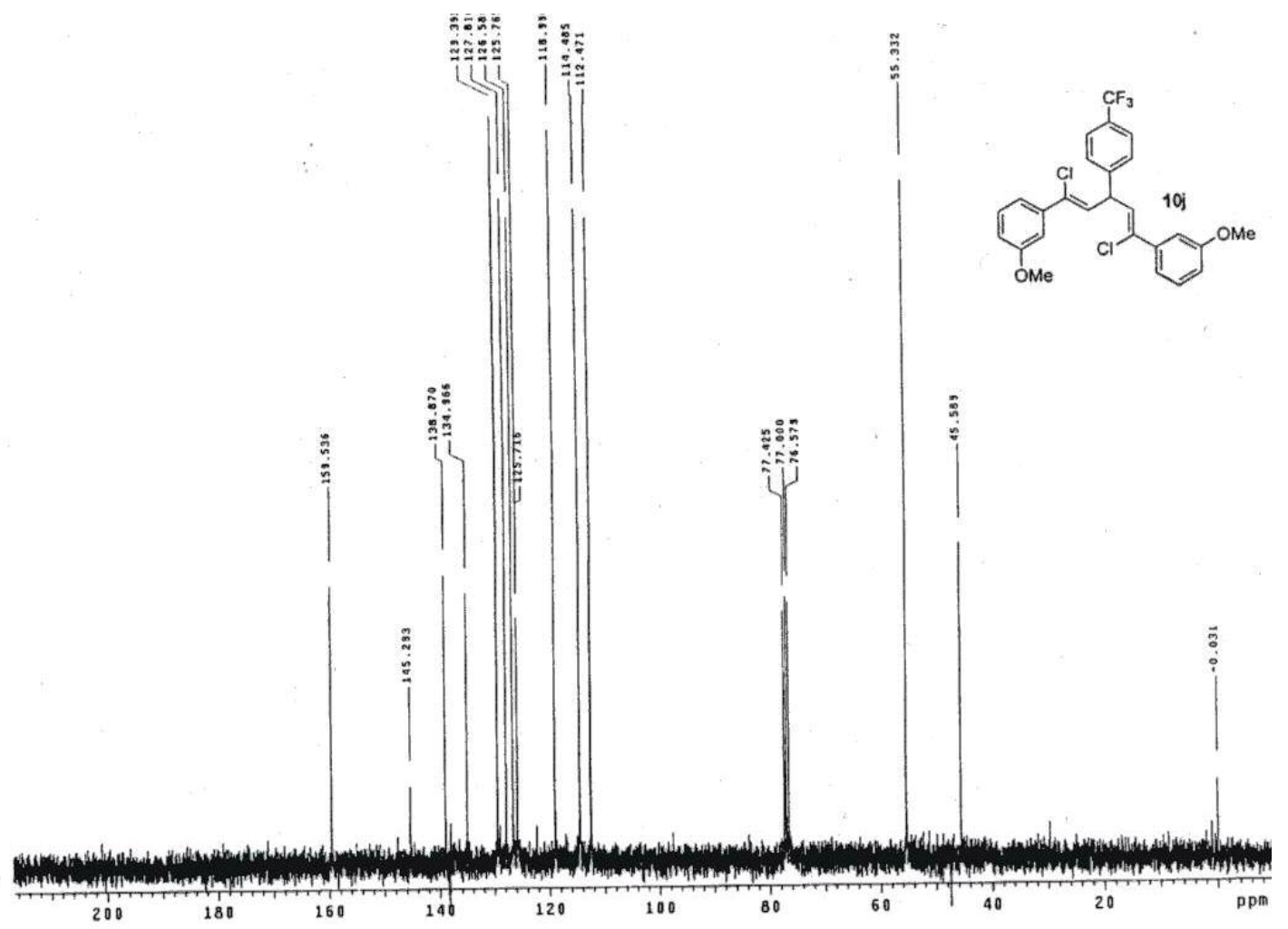




\section{${ }^{1}$ H- NMR spectrum of $10 k$}

/

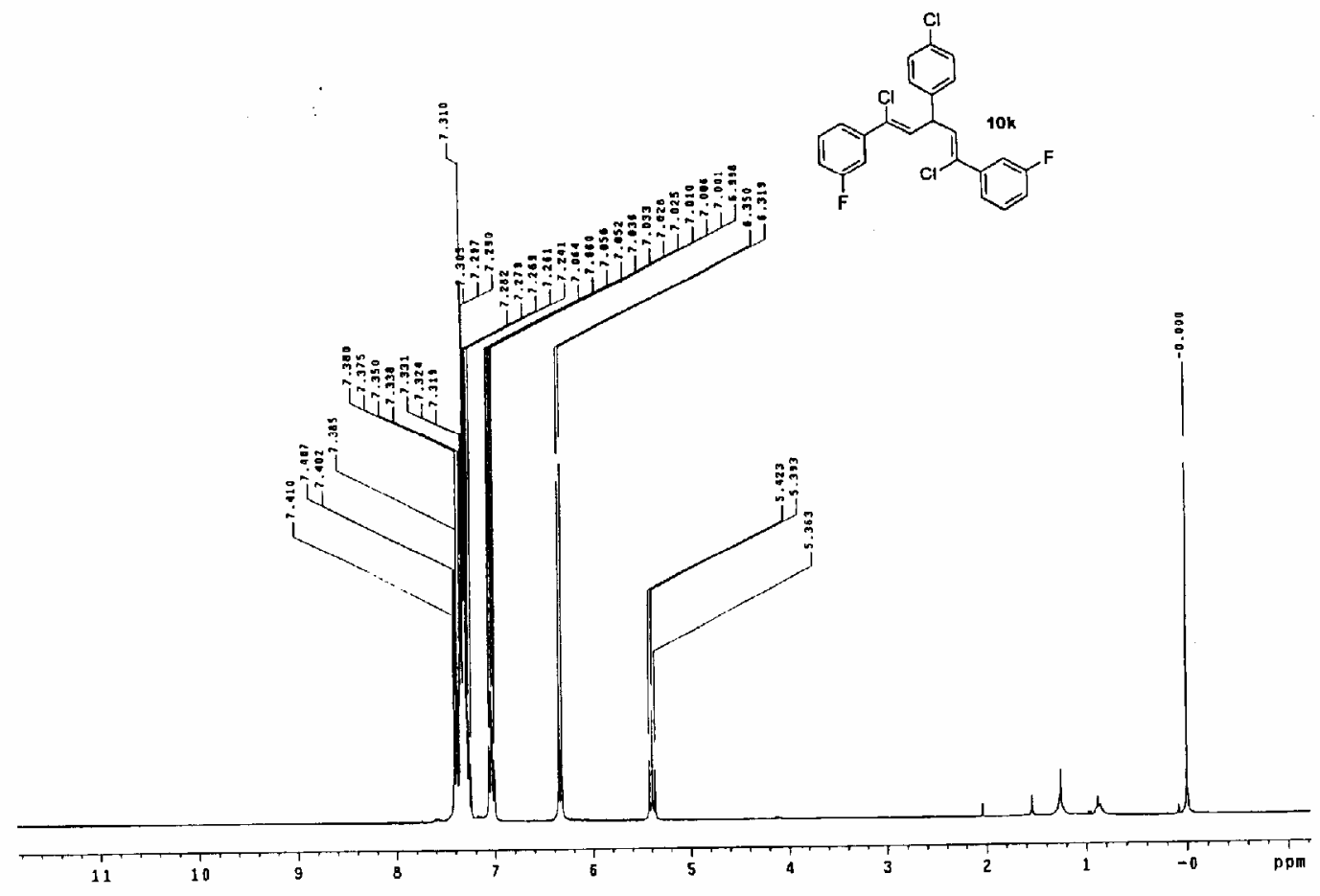


${ }^{13} \mathrm{C}$ - NMR spectrum of $10 \mathrm{k}$

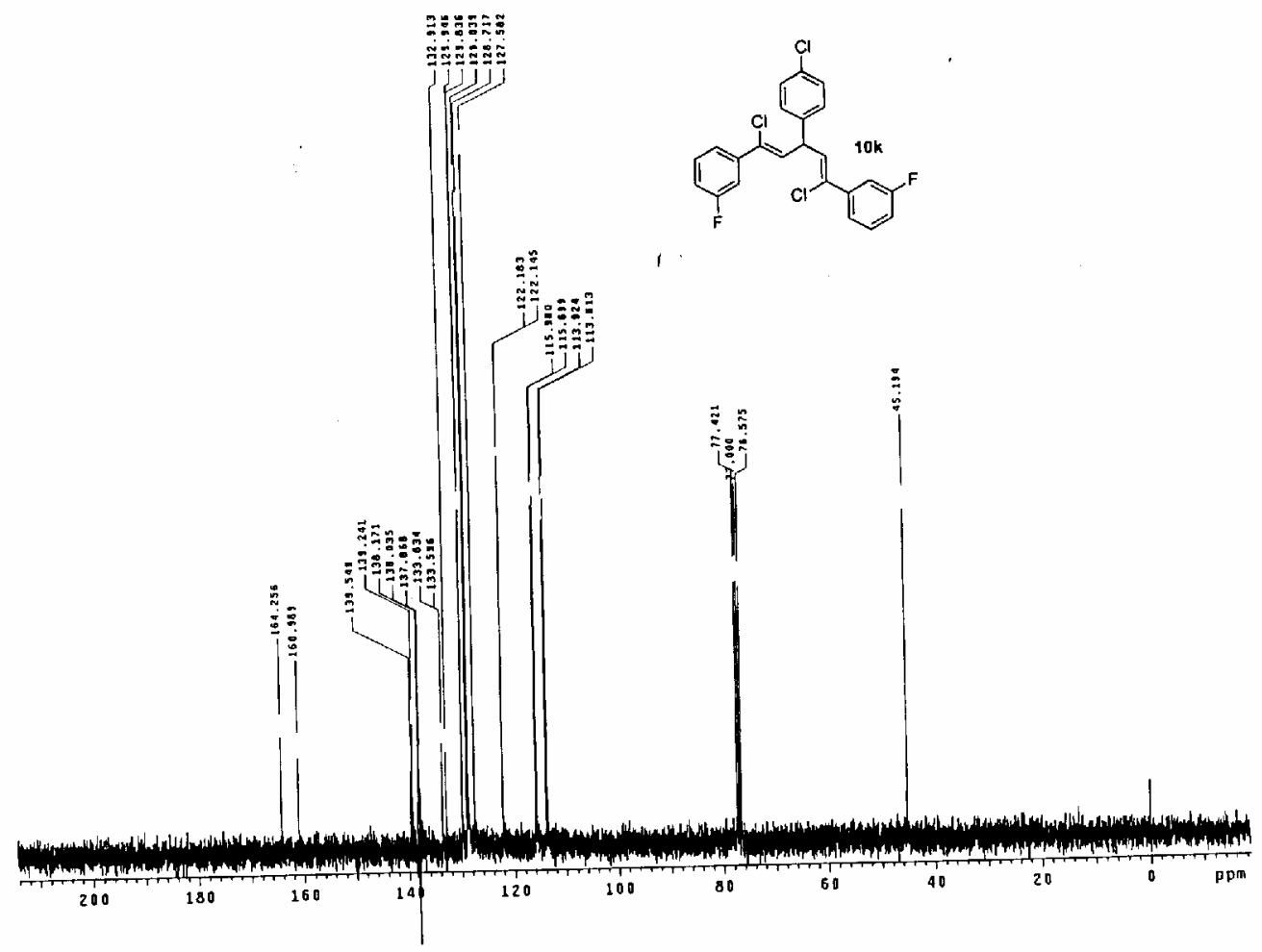




\section{${ }^{1} \mathrm{H}$ - NMR spectrum of $11 \mathrm{a}$}

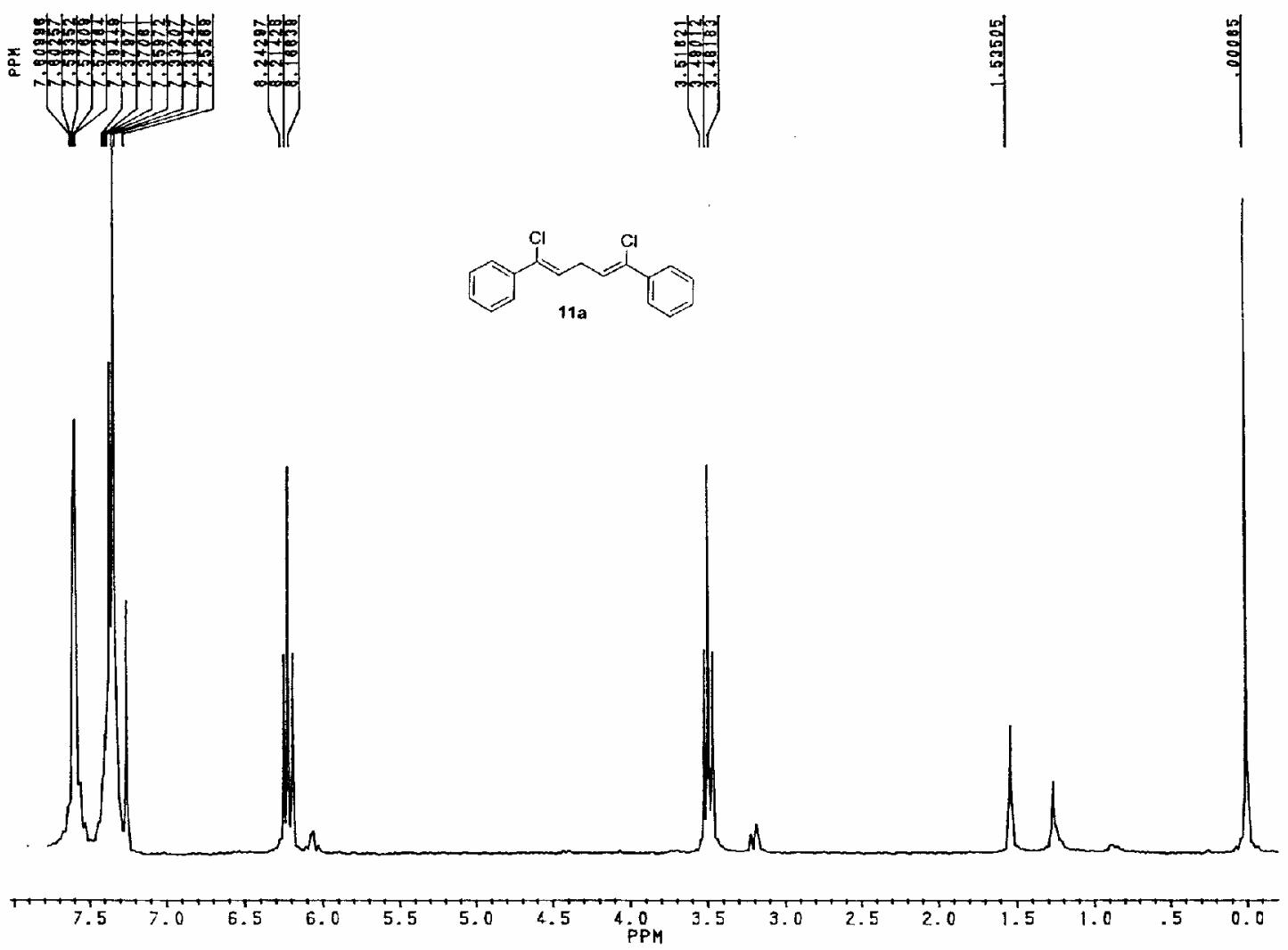




\section{${ }^{13} \mathrm{C}$ - NMR spectrum of $11 \mathrm{a}$}

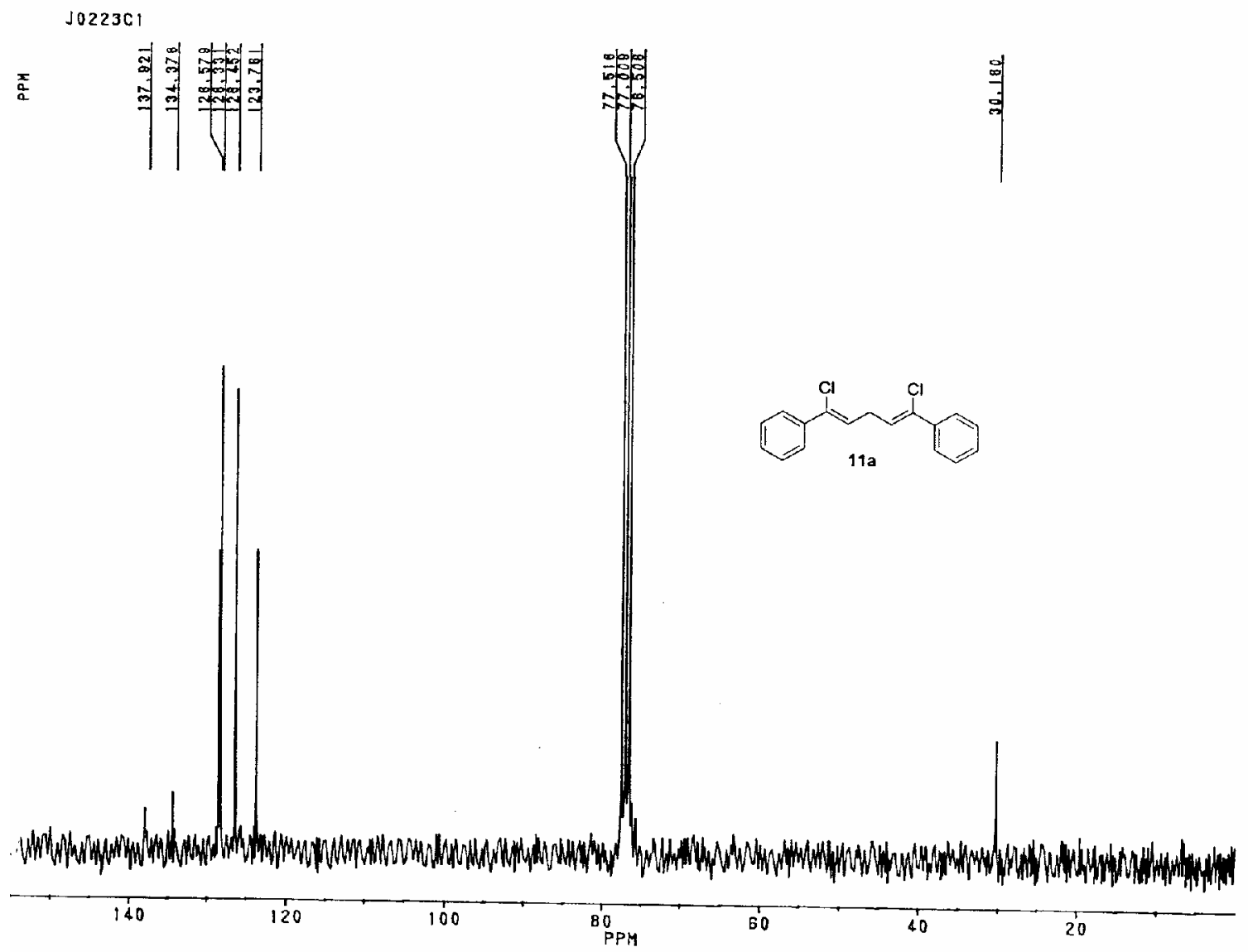




\section{${ }^{1} \mathrm{H}$ - NMR spectrum of $11 \mathrm{~b}$}

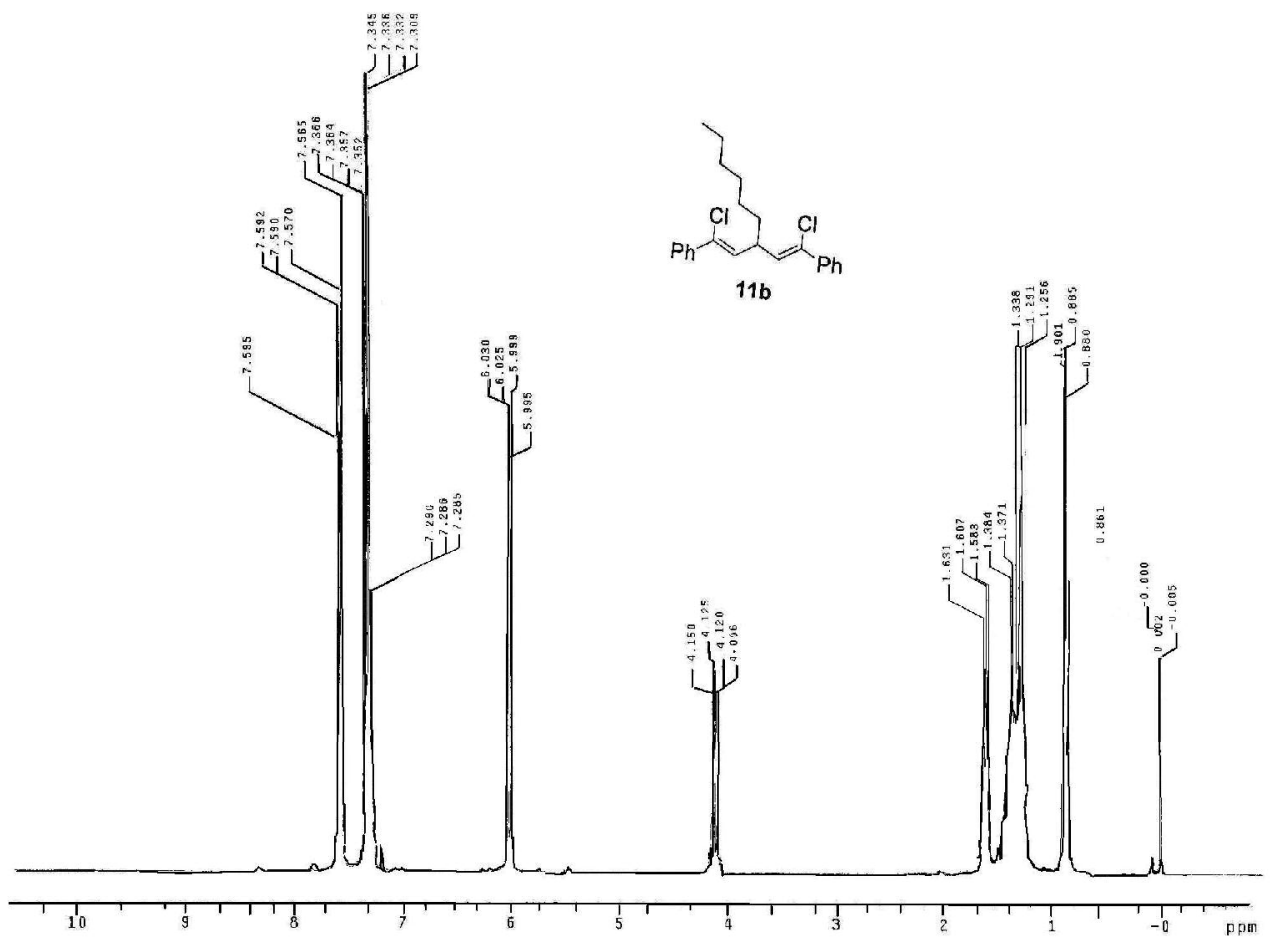




\section{${ }^{13} \mathrm{C}$-NMR spectrum of $11 \mathrm{~b}$}

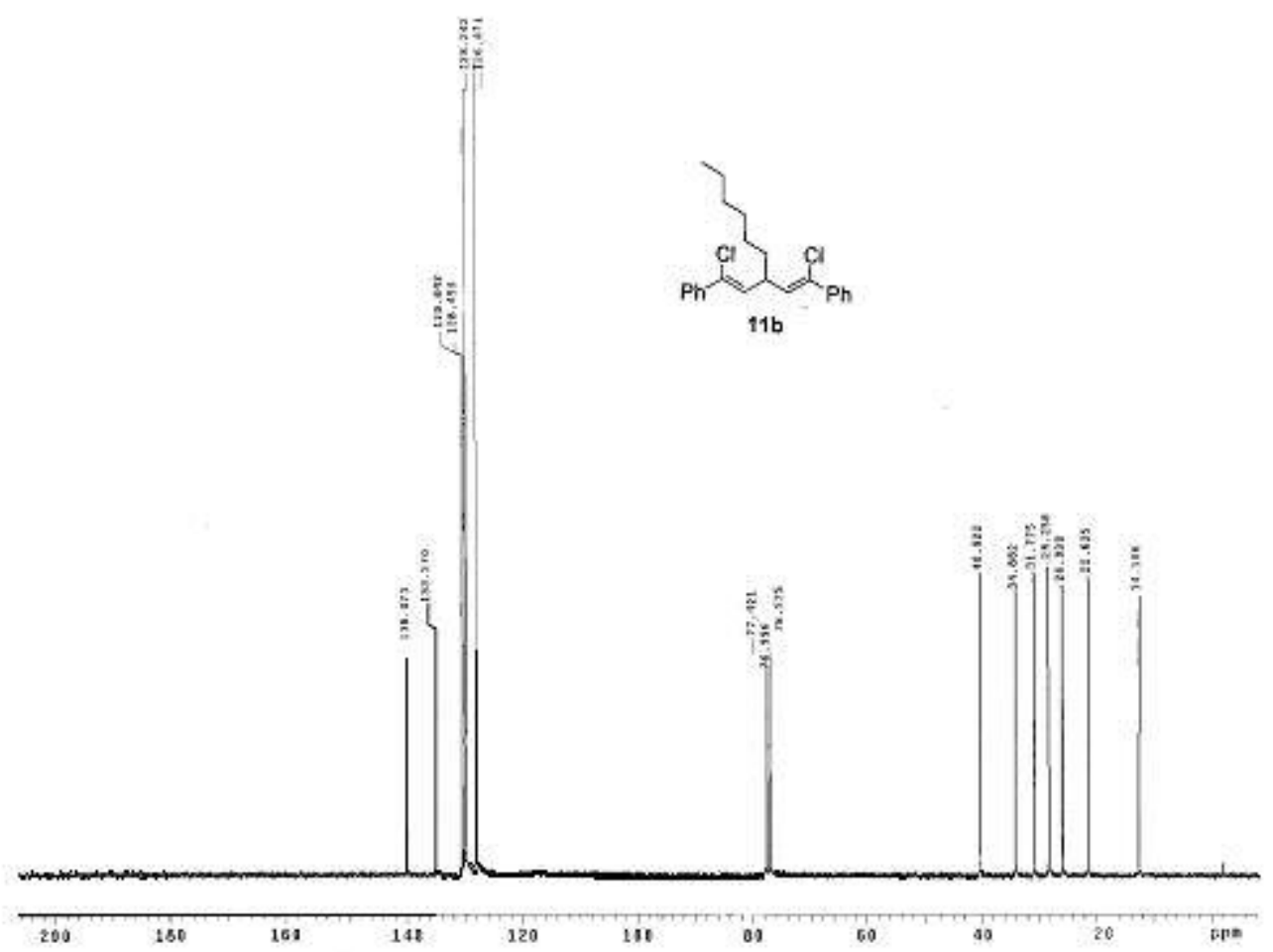




\section{${ }^{1} \mathrm{H}$ - NMR spectrum of 14}
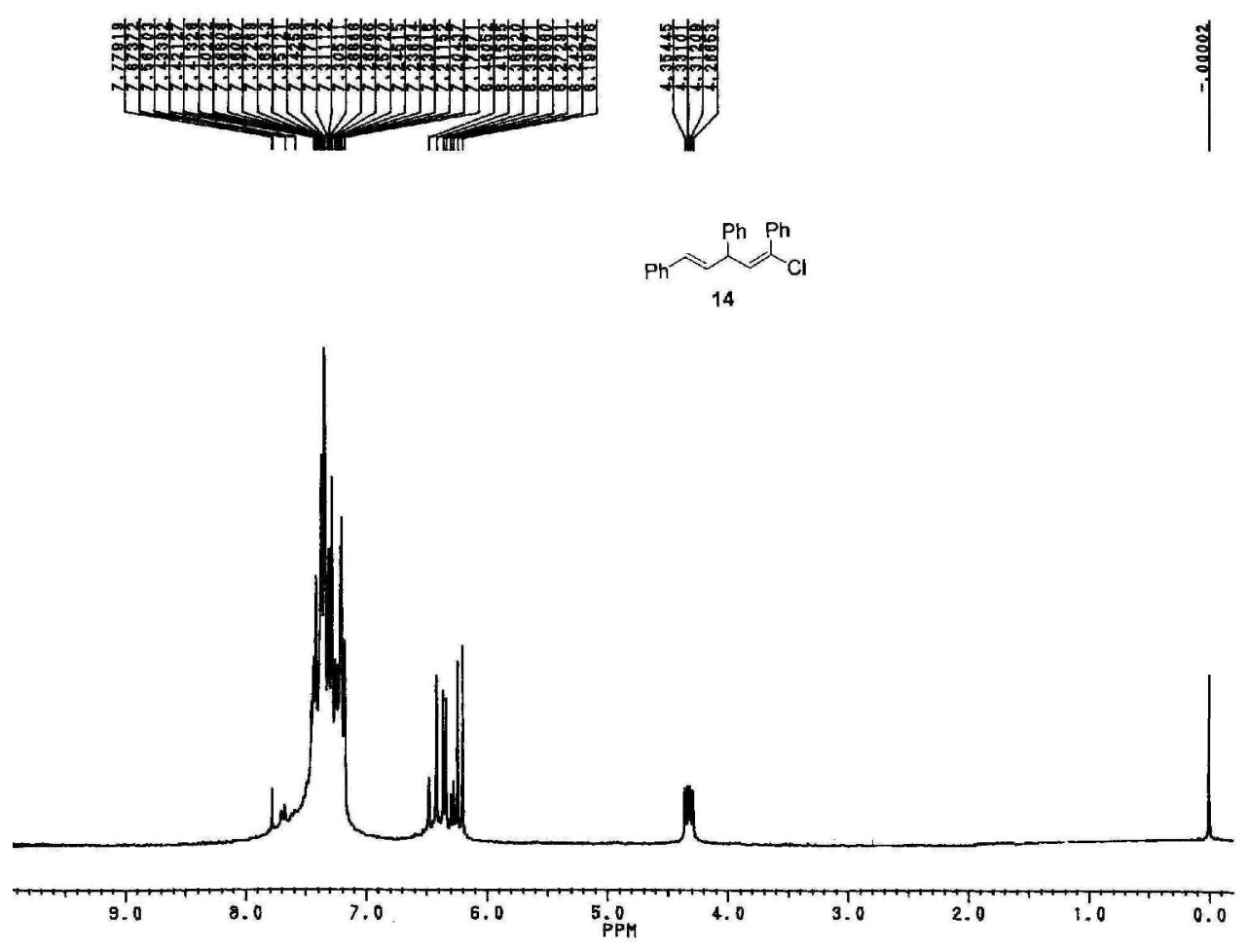
${ }^{13}$ C-NMR spectrum of 14
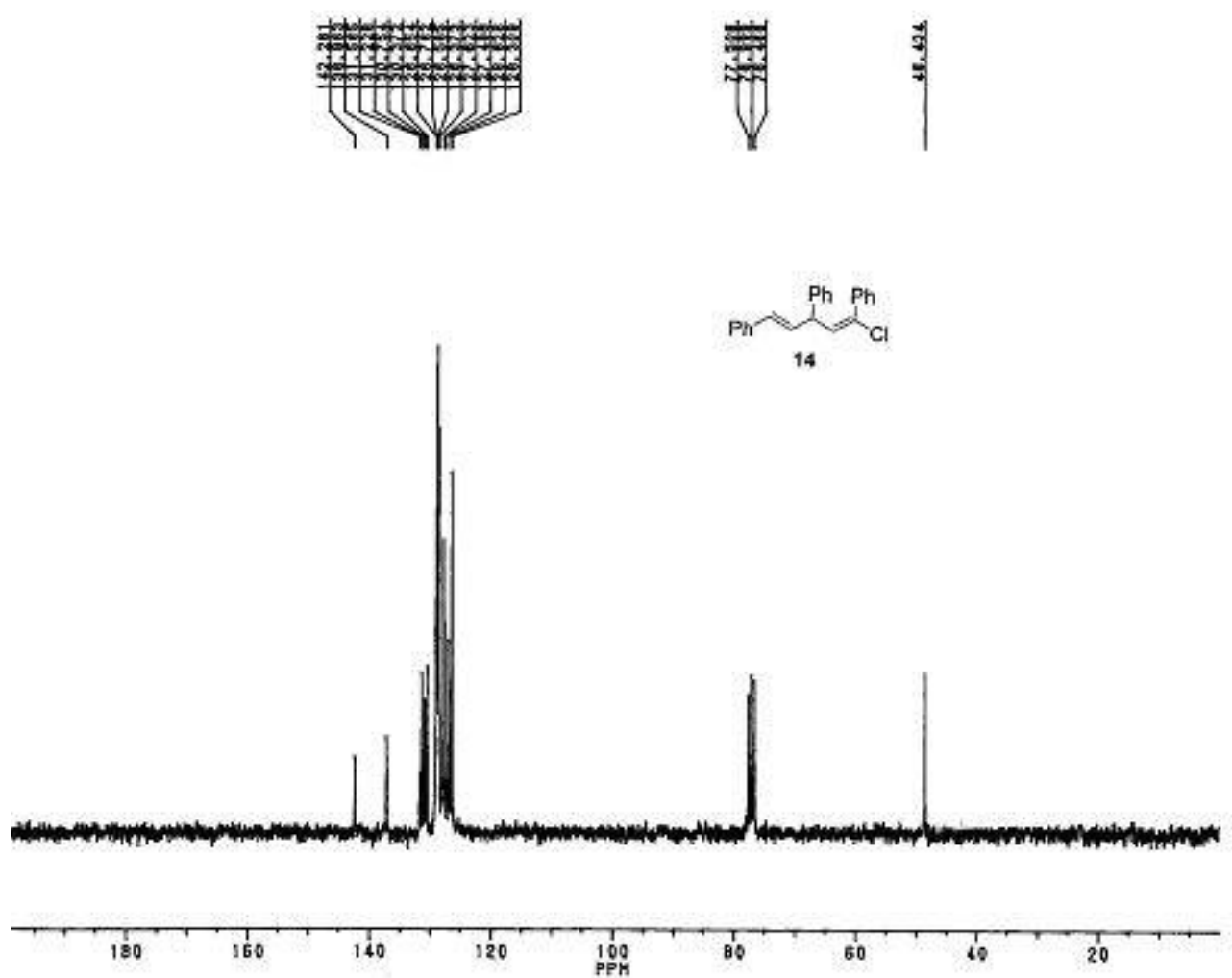